\title{
Comprehensive Search for New Phases and Compounds in Binary Alloy Systems Based on Platinum-Group Metals, Using a Computational First-Principles Approach
}

\author{
Gus L.W. Hart, ${ }^{1}$ Stefano Curtarolo, ${ }^{2, *}$ Thaddeus B. Massalski, ${ }^{3}$ and Ohad Levy ${ }^{2}$ \\ ${ }^{1}$ Department of Physics and Astronomy, Brigham Young University, Provo, Utah 84602, USA \\ ${ }^{2}$ Center for Materials Genomics, Department of Mechanical Engineering and Materials Science and Department of Physics, \\ Duke University, Durham, North Carolina 27708, USA \\ ${ }^{3}$ Materials Science, Engineering and Physics, Carnegie Mellon University, Pittsburgh, Pennsylvania 15213, USA
}

(Received 25 August 2013; published 30 December 2013)

\begin{abstract}
We report a comprehensive study of the binary systems of the platinum-group metals with the transition metals, using high-throughput first-principles calculations. These computations predict stability of new compounds in 28 binary systems where no compounds have been reported in the literature experimentally and a few dozen of as-yet unreported compounds in additional systems. Our calculations also identify stable structures at compound compositions that have been previously reported without detailed structural data and indicate that some experimentally reported compounds may actually be unstable at low temperatures. With these results, we construct enhanced structure maps for the binary alloys of platinum-group metals. These maps are much more complete, systematic, and predictive than those based on empirical results alone.
\end{abstract}

DOI: 10.1103/PhysRevX.3.041035

\section{INTRODUCTION}

The platinum-group metals (PGMs)—osmium, iridium, ruthenium, rhodium, platinum, and palladium-are immensely important in numerous technologies, but the experimental and computational data on their binary alloys still contain many gaps. Interest in PGMs is driven by their essential role in a wide variety of industrial applications, which is at odds with their high cost. The primary application of PGMs is in catalysis, where they are core ingredients in the chemical, petroleum, and automotive industries. They also extensively appear as alloying components in aeronautics and electronics applications. The use of platinum alloys in the jewelry industry also accounts for a sizable fraction of its worldwide consumption, about $30 \%$ over the last decade [1]. The importance and high cost of PGMs motivate numerous efforts directed at more effective usage or at the development of less-expensive alloy substitutes. Despite these efforts, there are still sizable gaps in the knowledge of the basic properties of PGMs and their alloys; many of the possible alloy compositions have not yet been studied, and there is a considerable difficulty in the application of thermodynamic experiments because they often require high temperatures or pressures and very long equilibration processes.

The possibility of predicting the existence of ordered structures in alloy systems from their starting components is a major challenge of current materials research.

\footnotetext{
*stefano@duke.edu
}

Published by the American Physical Society under the terms of the Creative Commons Attribution 3.0 License. Further distribution of this work must maintain attribution to the author(s) and the published article's title, journal citation, and DOI.
Subject Areas: Computational Physics, Materials Science

Empirical methods use experimental data to construct structure maps and make predictions based on clustering of simple physical parameters. Their usefulness depends on the availability of reliable data over the entire parameter space of components and stoichiometries. Advances in first-principles methods for the calculation of materials properties open the possibility to complement the experimental data by computational results. Indeed, many recent studies present such calculations of PGM alloy structures [2-25]. However, most of these studies consider a limited number of structures, at just a few stoichiometries of a single binary system or a few systems [3-17]. Some cluster expansion studies of specific binary systems include a larger set of structures, but limited to a single lattice type (usually fcc) [18-23]. Realizing the potential of firstprinciples calculations to complement the lacking, or only partial, empirical data requires high-throughput computational screening of large sets of materials, with structures spanning all lattice types and including, in addition, a considerable number of off-lattice structures [2,26-28]. Such large-scale screenings can be used to construct lowtemperature binary phase diagrams. They provide insight into trends in alloy properties and indicate the possible existence of hitherto unobserved compounds [27]. A few previous studies implemented this approach to binary systems of specific metals-hafnium, rhenium, rhodium, ruthenium, and technetium [24,25,29-31].

The capability to identify new phases is key to tuning the catalytic properties of PGM alloys and their utilization in new applications, or as reduced-cost or higher-activity substitutes in current applications. Even predicted phases that are difficult to access kinetically in the bulk may be exhibited in nanophase alloys [32] and could be used to increase the efficiency or the lifetimes of PGM catalysts. 
Given the potential payoff of uncovering such phases, we have undertaken a thorough examination of PGM binary phases with the transition metals, using the first-principles high-throughput (HT) framework AFLOW [33,34]. We find new potentially stable PGM phases in many binary systems, and comparing experimental data with our predictions, we construct enhanced Pettifor-type maps that demonstrate new ordering trends and compound-forming possibilities in these alloys.

\section{METHODS}

Computations of the low-temperature stability of the PGM-transition metal systems were carried out using the HT framework AFLOW [33,34]. For each of the 153 binary systems studied, we calculated the energies of more than 250 structures, including all the crystal structures reported for the system in the phase-diagram literature $[35,36]$ and additional structures from the AFLOWLIB database of prototypes and hypothetical hcp-, bcc-, and fcc-derivative superstructures [33]. A complete list of structures examined for each binary system can be found on the online repository [34,37] and in the supplemental material (see Ref. [38]). The low-temperature phase diagram of a system is constructed as the minimum formation enthalpy convex hull from these candidate structures, identifying the ordering trends in each alloy system and indicating the possible existence of previously unknown compounds. It should be noted that there is no guarantee that the true ground states of a system will be found among the common experimentally observed structures or among small-unit-cell derivative structures. However, even if it is impossible to rule out the existence of additional unexpected ground states, this protocol (searching many enumerated derivative structures [39] and exhaustively exploring experimentally reported ones) is expected to give a reasonable balance between high-throughput speed and scientific accuracy to determine miscibility (or lack thereof) in these alloys. In Ref. [2], it was shown that the probability of reproducing the correct ground state, if well defined and not ambiguous, is $\eta_{C}^{\star} \sim 96.7 \%$ ["reliability of the method," Eq. (3)].

The calculations of the structure energies were performed with the VASP software [40] with projectoraugmented-wave pseudopotentials [41] and the exchangecorrelation functionals parametrized by Perdew, Burke, and Ernzerhof for the generalized gradient approximation [42]. The energies were calculated at zero temperature and pressure, with spin polarization and without zeropoint motion or lattice vibrations. All crystal structures were fully relaxed (cell volume and shape and the basis atom coordinates inside the cell). Numerical convergence to about $1 \mathrm{meV} /$ atom was ensured by a high-energy cutoff (30\% higher than the maximum cutoff of both potentials) and a 6000-k-point, or higher, Monkhorst-Pack mesh [43].
The presented work comprises 39266 calculations, performed by using $1.82 \times 10^{6} \mathrm{CPU}$ hours on 2013 Intel Xeon E5 cores at $2.2 \mathrm{GHz}$. The calculations were carried out by extending the preexisting AFLOWLIB structure database [34] with additional calculations characterizing PGM alloys. Detailed information about all the examined structures can be found on the online repository [34,37], including input/output files, calculation parameters, geometry of the structures, energies, and formation energies. In addition, the reader can prepare phase diagrams (as in Figs. 5-19) linked to the appropriate structure URL locations. The Supplemental Material contains the whole set of geometrical and relaxed structures with $a b$ initio total energies and formation enthalpies (see Ref. [38]).

The analysis of formation enthalpy is, by itself, insufficient to compare alloy stability at different concentrations and their resilience toward high-temperature disorder. The formation enthalpy, $\Delta H\left(A_{x} B_{1-x}\right) \equiv H\left(A_{x} B_{1-x}\right)-x H(A)-$ $(1-x) H(B)$, represents the ordering strength of a mixture $A_{x} B_{1-x}$ against decomposition into its pure constituents at the appropriate proportion $x A$ and $(1-x) B(\Delta H$ is negative for compound-forming systems). However, it does not contain information about its resilience against disorder, which is captured by the entropy of the system. To quantify this resilience we define the entropic temperature

$$
T_{s} \equiv \max _{i}\left\{\frac{\Delta H\left(A_{x_{i}} B_{1-x_{i}}\right)}{k_{B}\left[x_{i} \log \left(x_{i}\right)+\left(1-x_{i}\right) \log \left(1-x_{i}\right)\right]}\right\},
$$

where $i$ counts all the stable compounds identified in the $A B$ binary system by the ab initio calculations, and the sign is chosen so that a positive temperature is needed for competing against compound stability. This definition assumes an ideal scenario [28], where the entropy is $S\left[\left\{x_{i}\right\}\right]=$ $-k_{B} \sum_{i} x_{i} \log \left(x_{i}\right)$. This first approximation should be considered as indicative of a trend (see Fig. 1 of Ref. [28] and Fig. 1), which might be modified somewhat by a systemspecific thorough analysis of the disorder. $T_{s}$ is a concentration-maximized formation enthalpy weighted by the inverse of its entropic contribution. It represents the deviation of a system convex hull from the purely entropic free-energy hull, $-T S(x)$, and hence the ability of its ordered phases to resist the deterioration into a temperaturedriven, entropically promoted, disordered binary mixture.

\section{HIGH-THROUGHPUT RESULTS}

We examine the 153 binary systems containing a PGM and a transition metal, including the PGM-PGM pairs (see Fig. 1). An exhaustive comparison of experimental and computational ground states is given in Tables I, II, III, IV, V, and VI. Convex hulls for systems that exhibit compounds are shown in the Appendix (Figs. 5-19). These results uncover 28 alloy systems reported as noncompound forming in the experimental literature but predicted computationally to have low-temperature stable 

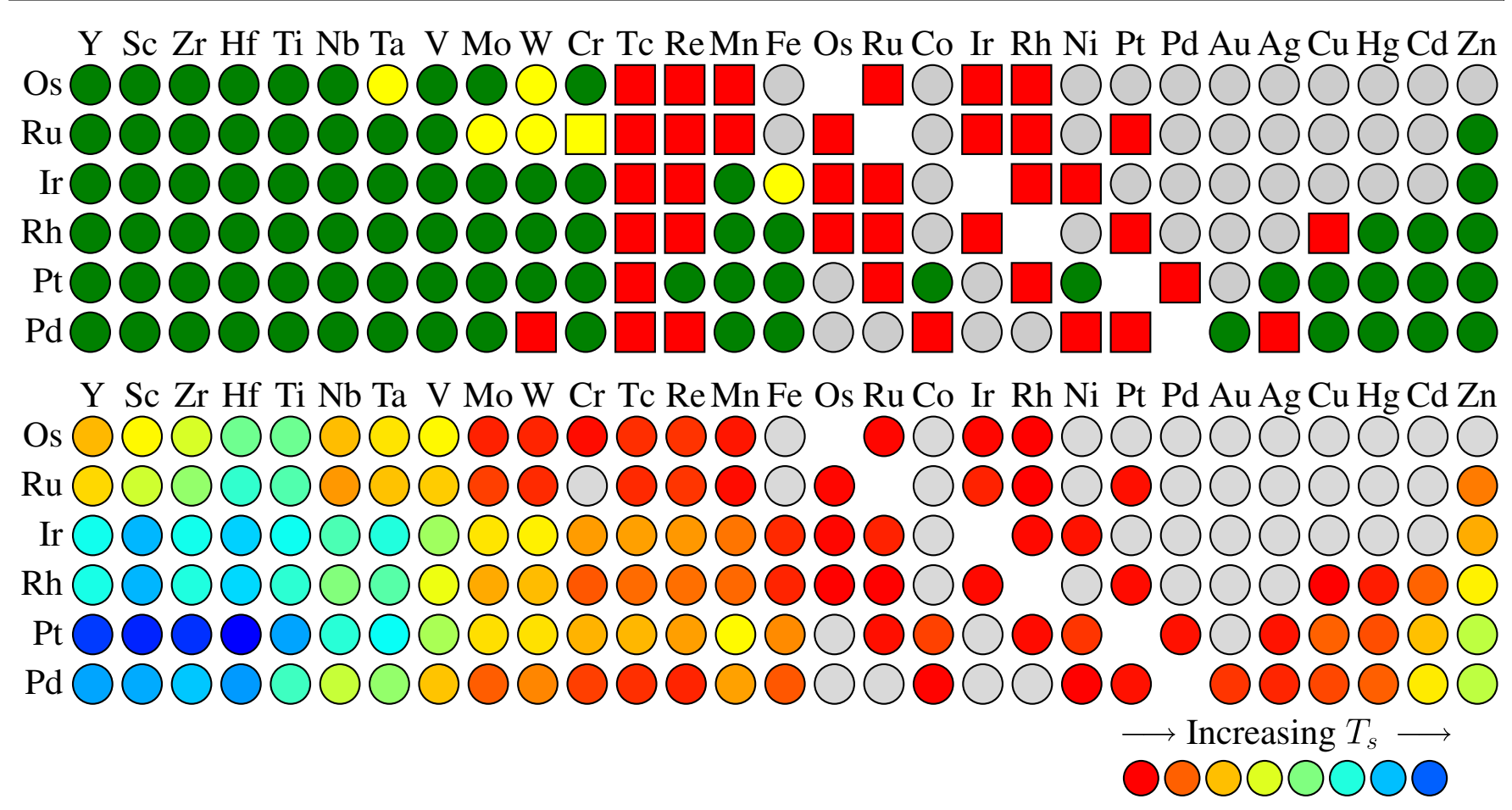

FIG. 1. Top panel: Compound-forming vs non-compound-forming systems as determined by experiment and computation. Circles indicate agreement between experiment and computation-green for compound-forming systems, gray for non-compound-forming systems. Yellow circles indicate systems reported in experiment to have disordered phases, for which low-energy compounds were found in this work. $\mathrm{Ru}-\mathrm{Cr}$ is the only system (yellow square) experimentally reported to include a disordered phase where no lowtemperature stable compounds were found. Red squares mark systems for which low-temperature compounds are found in computation but no compounds are reported in experiment. Bottom panel: $T_{s}$ for the binary systems in this work. Colors: From red to blue indicates the lowest to highest $T_{s}$.

compounds. Dozens of new compounds are also predicted in systems known to be compound forming.

The top panel of Fig. 1 gives a broad overview of the comparison of experiment and computation. Green circles (dark gray) indicate systems where experiment and computation agree that the system is compound forming. Light gray circles indicate agreement that the system is not compound forming. The elements along the axes of this diagram are listed according to their Pettifor $\chi$ parameter $[44,45]$, leading, as expected, to compound-forming and non-compound-forming systems separating rather cleanly into different broad regions of the diagram. Most of the compound-forming systems congregate in a large cluster on the left half of the diagram and in a second smaller cluster at the lower right corner.

The systems for which computation predicts compounds but experiment does not report any are marked by red squares. As is clear in the top panel of Fig. 1, these systems, which harbor potential new phases, occur near the boundary between the compound-forming and non-compoundforming regions of the diagram. They also fill in several isolated spots where experiment reports no compounds in the compound-forming region (e.g., $\mathrm{Pd}-\mathrm{W}$ and $\mathrm{Ag}-\mathrm{Pd}$ ), and they bridge the gap between the large cluster of compoundforming systems, on the left side of the panel, and the small island of such systems at its center. The computations also predict ordered structures in most systems reported only with disordered phases (yellow circles in top panel of Fig. 1). Two disordered phases, $\sigma$ and $\chi$, turn up in the experimental literature on PGM alloys. In the HT search, we included all ordered realizations of these phases (the prototypes $\mathrm{Al}_{12} \mathrm{Mg}_{17}$ and $\mathrm{Re}_{24} \mathrm{Ti}_{5}$ are ordered versions of the $\chi$ phase, and the $\sigma$ phase has 32 ordered realizations, denoted by $\sigma_{X X X X X}$, where $X=A, B$ ). In most of these systems, we find one of these corresponding ordered structures to be stable. The only exception is the Cr-Ru system, where the lowest-lying ordered phase is found just $4 \mathrm{meV} /$ atom above the elements' tieline (yellow square in Fig. 1). These results thus identify the low-temperature ordered compounds that underlie the reported disordered phases. The calculated compound-forming regions are considerably more extensive than reported by the available experimental data, identifying potential new systems for materials engineering.

The bottom panel of Fig. 1 ranks systems by their estimated entropic temperature $T_{s}$. Essentially, the (top panel) map, incorporating the computational data, corresponds to what would be observed at low temperatures, assuming thermodynamic equilibrium, whereas a map with only experimental data reports systems as compound 
TABLE I. Compounds observed in experiments or predicted by $a b$ initio calculations in Osmium binary alloys (structure prototype in parentheses; multiple entries denote different reported structures in the experiments or degenerate structures in the calculations). The "..." denotes no compounds. The superscript " $\star$ " denotes unobserved prototypes found in calculations $[2,13,25,27,29,31] . \Delta H$ are the formation enthalpies from the present study. The energy difference between reported and calculated structures or between the reported structure (unstable in the calculation) and a two-phase tieline is indicated as $\langle\cdot\rangle$.

=

Compounds

\begin{tabular}{|c|c|c|c|}
\hline & Experiments $[35,36]$ & Calculations & $\Delta H \mathrm{meV} / \mathrm{at}$. \\
\hline \multirow[t]{2}{*}{$\mathrm{Y}$} & $\mathrm{Os}_{2} \mathrm{Y}(\mathrm{C} 14)$ & $\mathrm{Os}_{2} \mathrm{Y}(\mathrm{C} 14)$ & -304 \\
\hline & $\mathrm{OsY}_{3}\left(\mathrm{D} 0_{11}\right)$ & $\mathrm{OsY}_{3}\left(\mathrm{D}_{11}\right)$ & -239 \\
\hline \multirow[t]{4}{*}{$\mathrm{Sc}$} & $\mathrm{Os}_{2} \mathrm{Sc}(\mathrm{C} 14)$ & $\mathrm{Os}_{2} \mathrm{Sc}(\mathrm{C} 14)$ & -390 \\
\hline & & $\mathrm{OsSc}_{2}\left(\mathrm{fcc}_{A B 2}^{[001]}\right)$ & -400 \\
\hline & $\mathrm{Os}_{4} \mathrm{Sc}_{11}\left(\mathrm{Ir}_{4} \mathrm{Sc}_{11}\right)$ & $\mathrm{Os}_{4} \mathrm{Sc}_{11}\left(\mathrm{Ir}_{4} \mathrm{Sc}_{11}\right)$ & -372 \\
\hline & $\mathrm{Os}_{7} \mathrm{Sc}_{44}\left(\mathrm{Mg}_{44} \mathrm{Rh}_{7}\right)$ & $\mathrm{Os}_{7} \mathrm{Sc}_{44}\left(\mathrm{Mg}_{44} \mathrm{Rh}_{7}\right)$ & -197 \\
\hline \multirow[t]{5}{*}{$\mathrm{Zr}$} & $\mathrm{Os}_{2} \mathrm{Zr}(\mathrm{C} 14)$ & $\mathrm{Os}_{2} \mathrm{Zr}(\mathrm{C} 14)$ & -388 \\
\hline & OsZr(B2) & OsZr(B2) & -524 \\
\hline & $\mathrm{Os}_{4} \mathrm{Zr}_{11}\left(\mathrm{Ir}_{4} \mathrm{Sc}_{11}\right)$ & $\mathrm{Os}_{4} \mathrm{Zr}_{11}\left(\mathrm{Ir}_{4} \mathrm{Sc}_{11}\right)$ & -29 \\
\hline & $\mathrm{Os}_{17} \mathrm{Zr}_{54}\left(\mathrm{Hf}_{54} \mathrm{Os}_{17}\right)$ & & $\langle 8\rangle$ \\
\hline & & $\mathrm{OsZr}_{4}\left(\mathrm{D} 1_{a}\right)$ & -220 \\
\hline
\end{tabular}

\begin{tabular}{crr}
\hline $\mathrm{Hf} \quad \mathrm{Hf}_{54} \mathrm{Os}_{17}\left(\mathrm{Hf}_{54} \mathrm{Os}_{17}\right)$ & & $\langle 20\rangle$ \\
$\mathrm{Hf}_{2} \mathrm{Os}\left(\mathrm{NiTi}_{2}\right)$ & $\langle 44\rangle$ \\
$\mathrm{HfOs}(\mathrm{B} 2)$ & $\mathrm{HfOs}(\mathrm{B} 2)$ & -709 \\
$\mathrm{HfOs}_{2}(\mathrm{C} 14)$ & & $\langle 66\rangle$ \\
\hline
\end{tabular}

\begin{tabular}{|c|c|c|c|}
\hline \multirow[t]{3}{*}{$\mathrm{Ti}$} & OsTi(B2) & OsTi(B2) & -714 \\
\hline & & $\mathrm{OsTi}_{2}(\mathrm{C} 49)$ & -515 \\
\hline & & $\mathrm{OsTi}_{3}\left(\mathrm{Mo}_{3} \mathrm{Ti}^{\star}\right)$ & -403 \\
\hline \multirow[t]{5}{*}{$\mathrm{Nb}$} & & $\mathrm{Nb}_{5} \mathrm{Os}\left(\mathrm{HfPd}_{5}^{\star}\right)$ & -200 \\
\hline & $\mathrm{Nb}_{3} \mathrm{Os}(\mathrm{A} 15)$ & $\mathrm{Nb}_{3} \mathrm{Os}(\mathrm{A} 15)$ & -275 \\
\hline & $\mathrm{Nb}_{0.6} \mathrm{Os}_{0.4}(\sigma)$ & $\mathrm{Nb}_{20} \mathrm{Os}_{10}\left(\sigma_{B A A B A}\right)$ & -274 \\
\hline & $\mathrm{Nb}_{0.4} \mathrm{Os}_{0.6}(\chi)$ & $\mathrm{Nb}_{12} \mathrm{Os}_{17}\left(\mathrm{Al}_{12} \mathrm{Mg}_{17}\right)$ & -247 \\
\hline & & $\mathrm{NbOs}_{3}\left(\mathrm{DO}_{24}\right)$ & -115 \\
\hline \multirow[t]{4}{*}{$\mathrm{Ta}$} & & $\mathrm{Os}_{2} \mathrm{Ta}\left(\mathrm{Ga}_{2} \mathrm{Hf}\right)$ & -205 \\
\hline & $\mathrm{Os}_{0.5} \mathrm{Ta}_{0.5}(\chi)$ & $\mathrm{Os}_{12} \mathrm{Ta}_{17}\left(\mathrm{Al}_{12} \mathrm{Mg}_{17}\right)$ & -313 \\
\hline & $\mathrm{Os}_{0.3} \mathrm{Ta}_{0.7}(\sigma)$ & $\mathrm{Os}_{10} \mathrm{Ta}_{20}\left(\sigma_{A B B A B}\right)$ & -335 \\
\hline & & $\mathrm{OsTa}_{3}(\mathrm{~A} 15)$ & -330 \\
\hline \multirow[t]{5}{*}{$\mathrm{V}$} & & $\mathrm{Os}_{3} \mathrm{~V}\left(\mathrm{Re}_{3} \mathrm{Ru}^{\star}\right)$ & -150 \\
\hline & & $\mathrm{Os}_{3} \mathrm{~V}_{5}\left(\mathrm{Ga}_{3} \mathrm{Pt}_{5}\right)$ & -350 \\
\hline & & $\mathrm{OsV}_{2}\left(\mathrm{C} 11_{b}\right)$ & -354 \\
\hline & $\mathrm{OsV}_{3}(\mathrm{~A} 15)$ & $\mathrm{OsV}_{3}\left(\mathrm{DO}_{3}\right)$ & $-361\langle 21\rangle$ \\
\hline & & $\mathrm{OsV}_{5}\left(\mathrm{Mo}_{5} \mathrm{Ti}^{\star}\right)$ & -253 \\
\hline
\end{tabular}

Mo $\quad \mathrm{Mo}_{3} \mathrm{Os}(\mathrm{A} 15)$
TABLE I. (Continued)

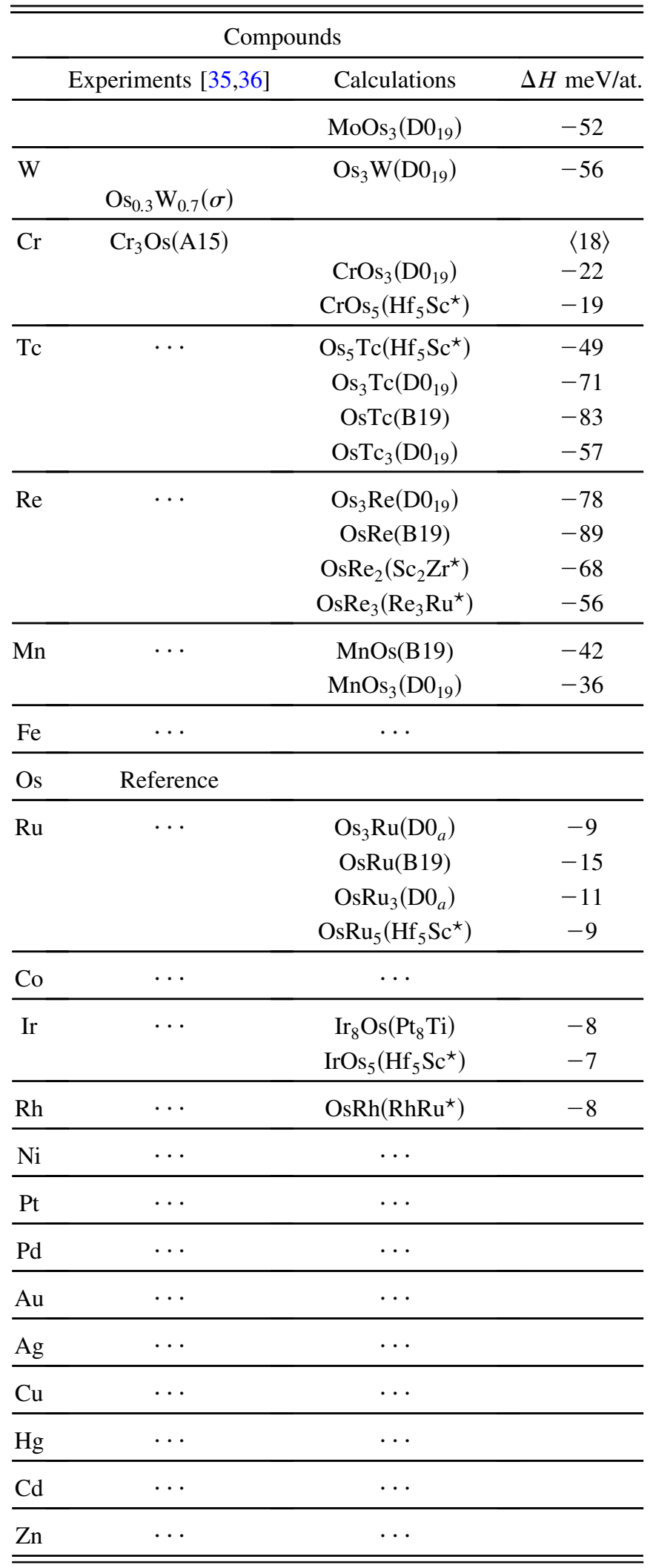


TABLE II. Compounds in Ruthenium binary alloys. The term (unkn.) denotes an unknown structure. All other symbols are as in Table I.

\begin{tabular}{|c|c|c|c|}
\hline \multicolumn{4}{|c|}{ Compounds } \\
\hline & Experiments $[35,36]$ & Calculations & $\Delta H \mathrm{meV} / \mathrm{at}$. \\
\hline \multirow[t]{5}{*}{ Y } & $\mathrm{Ru}_{2} \mathrm{Y}(\mathrm{C} 14)$ & $\mathrm{Ru}_{2} \mathrm{Y}(\mathrm{C} 14)$ & -313 \\
\hline & $\mathrm{Ru}_{2} \mathrm{Y}_{3}\left(\mathrm{Er}_{3} \mathrm{Ru}_{2}\right)$ & & $\langle 79\rangle$ \\
\hline & $\mathrm{Ru}_{25} \mathrm{Y}_{44}\left(\mathrm{Ru}_{25} \mathrm{Y}_{44}\right)$ & $\mathrm{Ru}_{25} \mathrm{Y}_{44}\left(\mathrm{Ru}_{25} \mathrm{Y}_{44}\right)$ & -342 \\
\hline & $\mathrm{Ru}_{2} \mathrm{Y}_{5}\left(\mathrm{C}_{2} \mathrm{Mn}_{5}\right)$ & $\mathrm{Ru}_{2} \mathrm{Y}_{5}\left(\mathrm{C}_{2} \mathrm{Mn}_{5}\right)$ & -334 \\
\hline & $\mathrm{RuY}_{3}\left(\mathrm{D} 0_{11}\right)$ & $\mathrm{RuY}_{3}\left(\mathrm{D} 0_{11}\right)$ & -307 \\
\hline \multirow[t]{7}{*}{$\mathrm{Sc}$} & $\mathrm{Ru}_{2} \mathrm{Sc}(\mathrm{C} 14)$ & $\mathrm{Ru}_{2} \mathrm{Sc}(\mathrm{C} 14)$ & -389 \\
\hline & $\operatorname{RuSc}(\mathrm{B} 2)$ & $\operatorname{RuSc}(\mathrm{B} 2)$ & -540 \\
\hline & $\mathrm{Ru}_{3} \mathrm{Sc}_{5}\left(\mathrm{D} 8_{8}\right)$ & & $\langle 42\rangle$ \\
\hline & $\mathrm{RuSc}_{2}\left(\mathrm{NiTi}_{2}\right)$ & $\operatorname{RuSc}_{2}\left(\mathrm{C} 11_{b}\right)$ & $-484\langle 84\rangle$ \\
\hline & $\mathrm{Ru}_{4} \mathrm{Sc}_{11}\left(\mathrm{Ir}_{4} \mathrm{Sc}_{11}\right)$ & $\mathrm{Ru}_{4} \mathrm{Sc}_{11}\left(\mathrm{Ir}_{4} \mathrm{Sc}_{11}\right)$ & -405 \\
\hline & $\mathrm{Ru}_{13} \mathrm{Sc}_{57}\left(\mathrm{Rh}_{13} \mathrm{Sc}_{57}\right)$ & & $\langle 10\rangle$ \\
\hline & $\mathrm{Ru}_{7} \mathrm{Sc}_{44}\left(\mathrm{Mg}_{44} \mathrm{Rh}_{7}\right)$ & $\mathrm{Ru}_{7} \mathrm{Sc}_{44}\left(\mathrm{Mg}_{44} \mathrm{Rh}_{7}\right)$ & -226 \\
\hline $\mathrm{Zr}$ & $\mathrm{RuZr}(\mathrm{B} 2)$ & $\operatorname{RuZr}(\mathrm{B} 2)$ & -644 \\
\hline \multirow[t]{2}{*}{$\mathrm{Hf}$} & $\operatorname{HfRu}(\mathrm{B} 2)$ & $\mathrm{HfRu}(\mathrm{B} 2)$ & -819 \\
\hline & $\mathrm{HfRu}_{2}$ (unkn.) & & \\
\hline \multirow[t]{3}{*}{$\mathrm{Ti}$} & RuTi(B2) & $\operatorname{RuTi(B2)}$ & -763 \\
\hline & & $\mathrm{RuTi}_{2}(\mathrm{C} 49)$ & -532 \\
\hline & & $\mathrm{RuTi}_{3}\left(\mathrm{Mo}_{3} \mathrm{Ti}^{\star}\right)$ & -401 \\
\hline \multirow[t]{4}{*}{$\mathrm{Nb}$} & & $\mathrm{Nb}_{8} \mathrm{Ru}\left(\mathrm{Pt}_{8} \mathrm{Ti}\right)$ & -117 \\
\hline & & $\mathrm{Nb}_{5} \mathrm{Ru}\left(\mathrm{Nb}_{5} \mathrm{Ru}^{\star}\right)$ & -172 \\
\hline & & $\mathrm{Nb}_{3} \mathrm{Ru}\left(\mathrm{L6}_{0}\right)$ & -222 \\
\hline & & $\mathrm{Nb}_{5} \mathrm{Ru}_{3}\left(\mathrm{Ga}_{3} \mathrm{Pt}_{5}\right)$ & -249 \\
\hline
\end{tabular}

$\mathrm{NbRu}$ (unkn.)

\begin{tabular}{|c|c|c|c|}
\hline & $\mathrm{NbRu}_{3}\left(\mathrm{~L}_{2}\right)$ & $\mathrm{Nb}_{3} \mathrm{Ru}_{5}\left(\mathrm{Ga}_{3} \mathrm{Pt}_{5}\right)$ & $\begin{array}{c}-240 \\
\langle 8\rangle\end{array}$ \\
\hline \multirow[t]{2}{*}{$\mathrm{Ta}$} & $\begin{array}{c}\mathrm{Ru}_{5} \mathrm{Ta}_{3} \text { (unkn.) } \\
\mathrm{RuTa} \text { (unkn.) }\end{array}$ & $\mathrm{Ru}_{5} \mathrm{Ta}_{3}\left(\mathrm{Ga}_{3} \mathrm{Pt}_{5}\right)$ & -332 \\
\hline & & $\begin{array}{c}\mathrm{Ru}_{3} \mathrm{Ta}_{5}\left(\mathrm{Ga}_{3} \mathrm{Pt}_{5}\right) \\
\operatorname{RuTa}_{3}\left(\mathrm{fcc}_{A B 3}^{[001]}\right) \\
\operatorname{RuTa}_{5}\left(\mathrm{Nb}_{5} \mathrm{Ru}^{\star}\right)\end{array}$ & $\begin{array}{l}-313 \\
-281 \\
-207 \\
\end{array}$ \\
\hline \multirow[t]{2}{*}{$\mathrm{V}$} & $\mathrm{RuV}(\mathrm{B} 11)$ & $\begin{array}{c}\mathrm{Ru}_{3} \mathrm{~V}\left(\mathrm{Re}_{3} \mathrm{Ru}^{\star}\right) \\
\mathrm{Ru}_{2} \mathrm{~V}(\mathrm{C} 37)\end{array}$ & $\begin{array}{l}-145 \\
-192 \\
\langle 28\rangle\end{array}$ \\
\hline & & $\begin{array}{c}\mathrm{Ru}_{3} \mathrm{~V}_{5}\left(\mathrm{Ga}_{3} \mathrm{Pt}_{5}\right) \\
\mathrm{RuV}_{2}\left(\mathrm{Cl}_{b}\right) \\
\mathrm{RuV}_{3}\left(\mathrm{Mo}_{3} \mathrm{Ti}^{\star}\right) \\
\mathrm{RuV}_{4}\left(\mathrm{Di}_{a}\right) \\
\mathrm{RuV}_{5}\left(\mathrm{Nb}_{5} \mathrm{Ru}^{\star}\right) \\
\mathrm{RuV}_{8}\left(\mathrm{Pt}_{8} \mathrm{Ti}\right)\end{array}$ & $\begin{array}{l}-313 \\
-321 \\
-296 \\
-262 \\
-230 \\
-154\end{array}$ \\
\hline Мo & $\mathrm{Mo}_{0.6} \mathrm{Ru}_{0.4}(\sigma)$ & $\mathrm{Mo}_{14} \mathrm{Ru}_{16}\left(\sigma_{A A B A B}\right)$ & -116 \\
\hline $\mathrm{W}$ & $\mathrm{Ru}_{0.4} \mathrm{~W}_{0.6}(\sigma)$ & $\mathrm{Ru}_{3} \mathrm{~W}\left(\mathrm{D} 0_{19}\right)$ & -65 \\
\hline
\end{tabular}

TABLE II. (Continued)

\begin{tabular}{|c|c|c|c|}
\hline \multicolumn{4}{|c|}{ Compounds } \\
\hline & Experiments $[35,36]$ & Calculations & $\Delta H \mathrm{meV} / \mathrm{at}$. \\
\hline $\mathrm{Cr}$ & $\mathrm{Cr}_{0.7} \mathrm{Ru}_{0.3}(\sigma)$ & $\cdots$ & \\
\hline $\mathrm{Tc}$ & $\ldots$ & $\begin{array}{c}\operatorname{Ru}_{3} \mathrm{Tc}\left(\mathrm{DO}_{19}\right) \\
\operatorname{RuTc}(\mathrm{B} 19) \\
\operatorname{RuTc}_{3}\left(\mathrm{DO}_{19}\right) \\
\mathrm{RuTc}_{5}\left(\mathrm{RuTc}_{5}{ }^{\star}\right)\end{array}$ & $\begin{array}{l}-63 \\
-73 \\
-47 \\
-32 \\
\end{array}$ \\
\hline $\mathrm{Re}$ & $\cdots$ & $\begin{array}{c}\operatorname{Re}_{3} \operatorname{Ru}\left(\operatorname{Re}_{3} \mathrm{Ru}^{\star}\right) \\
\operatorname{ReRu}(\mathrm{B} 19) \\
\operatorname{ReRu}_{3}\left(\mathrm{D} 0_{19}\right) \\
\end{array}$ & $\begin{array}{l}-53 \\
-86 \\
-80 \\
\end{array}$ \\
\hline $\mathrm{Mn}$ & $\cdots$ & $\mathrm{Mn}_{24} \mathrm{Ru}_{5}\left(\mathrm{Re}_{24} \mathrm{Ti}_{5}\right)$ & -18 \\
\hline $\mathrm{Fe}$ & $\cdots$ & $\cdots$ & \\
\hline Os & $\cdots$ & $\begin{array}{c}\mathrm{Os}_{3} \mathrm{Ru}\left(\mathrm{D0} 0_{a}\right) \\
\mathrm{OsRu}(\mathrm{B} 19) \\
\mathrm{OsRu}_{3}\left(\mathrm{D} 0_{a}\right) \\
\mathrm{OsRu}_{5}\left(\mathrm{Hf}_{5} \mathrm{Sc}^{\star}\right)\end{array}$ & $\begin{array}{l}-9 \\
-15 \\
-11 \\
-9\end{array}$ \\
\hline $\mathrm{Ru}$ & Reference & & \\
\hline $\mathrm{Co}$ & $\cdots$ & $\cdots$ & \\
\hline $\mathrm{Ir}$ & $\cdots$ & $\begin{array}{c}\mathrm{Ir}_{8} \mathrm{Ru}\left(\mathrm{Pt}_{8} \mathrm{Ti}\right) \\
\mathrm{Ir}_{3} \mathrm{Ru}\left(\mathrm{Ll}_{2}\right) \\
\operatorname{IrRu}(\mathrm{B} 19) \\
\operatorname{IrRu}_{2}\left(\mathrm{Ir}_{2} \mathrm{Tc}^{\star}\right) \\
\operatorname{IrRu}_{3}\left(\mathrm{D} 0_{19}\right) \\
\mathrm{IrRu}_{5}\left(\mathrm{Hf}_{5} \mathrm{Sc}^{\star}\right)\end{array}$ & $\begin{array}{l}-20 \\
-34 \\
-49 \\
-54 \\
-53 \\
-37 \\
\end{array}$ \\
\hline $\mathrm{Rh}$ & $\cdots$ & $\begin{array}{c}\mathrm{Rh}_{8} \mathrm{Ru}\left(\mathrm{Pt}_{8} \mathrm{Ti}\right) \\
\mathrm{RhRu}\left(\mathrm{RhRu}^{\star}\right) \\
\mathrm{RhRu}_{2}\left(\mathrm{RhRu}_{2}{ }^{\star}\right) \\
\mathrm{RhRu}_{5}\left(\mathrm{RhRu}_{5}^{\star}\right)\end{array}$ & $\begin{array}{l}-2 \\
-8 \\
-6 \\
-3\end{array}$ \\
\hline $\mathrm{Ni}$ & $\cdots$ & $\ldots$ & \\
\hline $\mathrm{Pt}$ & $\cdots$ & PtRu(CdTi) & -33 \\
\hline $\mathrm{Pd}$ & $\cdots$ & $\cdots$ & \\
\hline $\mathrm{Au}$ & $\cdots$ & $\cdots$ & \\
\hline $\mathrm{Ag}$ & $\cdots$ & $\cdots$ & \\
\hline $\mathrm{Cu}$ & $\cdots$ & $\cdots$ & \\
\hline $\mathrm{Hg}$ & $\cdots$ & $\cdots$ & \\
\hline $\mathrm{Cd}$ & $\cdots$ & $\cdots$ & \\
\hline $\mathrm{Zn}$ & $\mathrm{RuZn}_{6}\left(\mathrm{RuZn}_{6}\right)$ & $\begin{array}{c}\operatorname{RuZn}_{3}\left(\mathrm{L1}_{2}\right) \\
\operatorname{RuZn}_{6}\left(\mathrm{RuZn}_{6}\right)\end{array}$ & $\begin{array}{l}-150 \\
-132\end{array}$ \\
\hline
\end{tabular}


TABLE III. Compounds in Iridium binary alloys. The superscript "§" denotes relaxation of one prototype into another and a " $\dagger$ " denotes new prototypes described in Table VII. The other symbols are as in Table II.

\begin{tabular}{|c|c|c|c|}
\hline & \multicolumn{2}{|c|}{ Compounds } & \multirow[b]{2}{*}{$\Delta H \mathrm{meV} / \mathrm{at}$} \\
\hline & Experiments $[35,36]$ & Calculations & \\
\hline \multirow[t]{7}{*}{ Y } & $\mathrm{Ir}_{3} \mathrm{Y}\left(\mathrm{PuNi}_{3}\right)$ & & $\langle 21\rangle$ \\
\hline & $\mathrm{Ir}_{2} \mathrm{Y}(\mathrm{C} 15)$ & $\mathrm{Ir}_{2} \mathrm{Y}(\mathrm{C} 15)$ & -803 \\
\hline & $\operatorname{IrY}(\mathrm{B} 2)$ & $\operatorname{IrY}(\mathrm{B} 2)$ & -787 \\
\hline & $\mathrm{Ir}_{2} \mathrm{Y}_{3}\left(\mathrm{Rh}_{2} \mathrm{Y}_{3}\right)$ & & $\langle 12\rangle$ \\
\hline & $\mathrm{Ir}_{3} \mathrm{Y}_{5}\left(\mathrm{Pu}_{5} \mathrm{Rh}_{3}\right)$ & $\mathrm{Ir}_{3} \mathrm{Y}_{5}\left(\mathrm{Pu}_{5} \mathrm{Rh}_{3}\right)$ & -772 \\
\hline & $\mathrm{Ir}_{2} \mathrm{Y}_{5}\left(\mathrm{C}_{2} \mathrm{Mn}_{5}\right)$ & $\mathrm{Ir}_{2} \mathrm{Y}_{5}\left(\mathrm{C}_{2} \mathrm{Mn}_{5}\right)$ & -640 \\
\hline & $\mathrm{IrY}_{3}\left(\mathrm{D} 0_{11}\right)$ & $\mathrm{IrY}_{3}\left(\mathrm{D} 0_{11}\right)$ & -564 \\
\hline \multirow[t]{8}{*}{$\mathrm{Sc}$} & & $\mathrm{Ir}_{7} \mathrm{Sc}\left(\mathrm{CuPt}_{7}\right)$ & -352 \\
\hline & $\mathrm{Ir}_{3} \mathrm{Sc}\left(\mathrm{L1}_{2}\right)$ & & $\langle 7\rangle$ \\
\hline & $\mathrm{Ir}_{2} \mathrm{Sc}(\mathrm{C} 15)$ & $\mathrm{Ir}_{2} \mathrm{Sc}(\mathrm{C} 14)$ & $-783\langle 35\rangle$ \\
\hline & $\operatorname{IrSc}(\mathrm{B} 2)$ & $\operatorname{IrSc}(\mathrm{B} 2)$ & -1032 \\
\hline & $\operatorname{IrSc}_{2}\left(\mathrm{NiTi}_{2}\right)$ & & $\langle 26\rangle$ \\
\hline & $\mathrm{Ir}_{4} \mathrm{Sc}_{11}\left(\mathrm{Ir}_{4} \mathrm{Sc}_{11}\right)$ & $\mathrm{Ir}_{4} \mathrm{Sc}_{11}\left(\mathrm{Ir}_{4} \mathrm{Sc}_{11}\right)$ & -686 \\
\hline & $\mathrm{Ir}_{13} \mathrm{Sc}_{57}\left(\mathrm{Rh}_{13} \mathrm{Sc}_{57}\right)$ & & $\langle 2\rangle$ \\
\hline & $\mathrm{Ir}_{7} \mathrm{Sc}_{44}\left(\mathrm{Mg}_{44} \mathrm{Rh}_{7}\right)$ & $\mathrm{Ir}_{7} \mathrm{Sc}_{44}\left(\mathrm{Mg}_{44} \mathrm{Rh}_{7}\right)$ & -369 \\
\hline \multirow[t]{6}{*}{$\mathrm{Zr}$} & $\mathrm{Ir}_{3} \mathrm{Zr}\left(\mathrm{L1}_{2}\right)$ & $\mathrm{Ir}_{3} \mathrm{Zr}\left(\mathrm{L} 1_{2}\right)$ & -709 \\
\hline & $\mathrm{Ir}_{2} \mathrm{Zr}(\mathrm{C} 15)$ & $\mathrm{Ir}_{2} \mathrm{Zr}\left(\mathrm{Ga}_{2} \mathrm{Hf}\right)$ & $-766\langle 87\rangle$ \\
\hline & IrZr(NiTi) & $\operatorname{IrZr}(\mathrm{NiTi})$ & -830 \\
\hline & $\mathrm{Ir}_{3} \mathrm{Zr}_{5}\left(\mathrm{Ir}_{3} \mathrm{Zr}_{5}\right)$ & $\mathrm{Ir}_{3} \mathrm{Zr}_{5}\left(\mathrm{Ir}_{3} \mathrm{Zr}_{5}\right)$ & -732 \\
\hline & $\mathrm{IrZr}_{2}(\mathrm{C} 16)$ & $\mathrm{IrZr}_{2}(\mathrm{C} 37)$ & $-668\langle 13\rangle$ \\
\hline & $\mathrm{IrZr}_{3}\left(\mathrm{SV}_{3}\right)$ & $\mathrm{IrZr}_{3}\left(\mathrm{SV}_{3}\right)$ & -519 \\
\hline \multirow[t]{5}{*}{$\mathrm{Hf}$} & $\mathrm{Hf}_{2} \mathrm{Ir}\left(\mathrm{NiTi}_{2}\right)$ & $\mathrm{Hf}_{2} \mathrm{Ir}(\mathrm{C} 37)$ & $-750\langle 31\rangle$ \\
\hline & $\mathrm{Hf}_{5} \mathrm{Ir}_{3}\left(\mathrm{D} 8_{8} / \mathrm{Ir}_{5} \mathrm{Zr}_{3}\right)$ & $\mathrm{Hf}_{5} \mathrm{Ir}_{3}\left(\mathrm{Ir}_{5} \mathrm{Zr}_{3}\right)$ & $-814\langle 14\rangle$ \\
\hline & HfIr (unkn.) & HfIr(B27) & -949 \\
\hline & & $\mathrm{HfIr}_{2}\left(\mathrm{Ga}_{2} \mathrm{Hf}\right)$ & -872 \\
\hline & $\mathrm{HfIr}_{3}\left(\mathrm{L1}_{2}\right)$ & $\operatorname{HfIr}_{3}\left(\mathrm{L1}_{2}\right)$ & -800 \\
\hline \multirow[t]{7}{*}{$\mathrm{Ti}$} & & $\mathrm{Ir}_{7} \mathrm{Ti}\left(\mathrm{CuPt}_{7}\right)$ & -369 \\
\hline & $\mathrm{Ir}_{3} \operatorname{Ti}\left(\mathrm{L1}_{2}\right)$ & $\mathrm{Ir}_{3} \operatorname{Ti}\left(\mathrm{L1}_{2}\right)$ & -716 \\
\hline & & $\mathrm{Ir}_{2} \mathrm{Ti}\left(\mathrm{Ga}_{2} \mathrm{Hf}\right)$ & -779 \\
\hline & & $\mathrm{Ir}_{5} \mathrm{Ti}_{3}\left(\mathrm{Ga}_{3} \mathrm{Pt}_{5}\right)$ & -809 \\
\hline & IrTi (unkn.) & $\operatorname{IrTi}\left(\mathrm{L1}_{0}\right)$ & -847 \\
\hline & & $\operatorname{IrTi}_{2}\left(\mathrm{C} 11_{b}\right)$ & -712 \\
\hline & $\mathrm{IrTi}_{3}(\mathrm{~A} 15)$ & $\mathrm{IrTi}_{3}(\mathrm{~A} 15)$ & -566 \\
\hline \multirow[t]{4}{*}{$\mathrm{Nb}$} & $\mathrm{Ir}_{3} \mathrm{Nb}\left(\mathrm{L1}_{2}\right)$ & $\mathrm{Ir}_{3} \mathrm{Nb}\left(\mathrm{Co}_{3} \mathrm{~V}\right)$ & $-628\langle 9\rangle$ \\
\hline & $\operatorname{IrNb}\left(\mathrm{L1}_{0} / \mathrm{IrTa}\right)$ & $\operatorname{IrNb}\left(\mathrm{L1}_{0}\right)$ & $-542\langle 2\rangle$ \\
\hline & $\mathrm{Ir}_{0.37} \mathrm{Nb}_{0.63}(\sigma)$ & $\mathrm{Ir}_{2} \mathrm{Nb}_{5}\left(\sigma_{A B B A B}\right)$ & -484 \\
\hline & $\mathrm{IrNb}_{3}(\mathrm{~A} 15)$ & $\mathrm{IrNb}_{3}(\mathrm{~A} 15)$ & -433 \\
\hline \multirow[t]{5}{*}{$\mathrm{Ta}$} & $\mathrm{Ir}_{3} \mathrm{Ta}\left(\mathrm{L} 1_{2}\right)$ & $\mathrm{Ir}_{3} \mathrm{Ta}\left(\mathrm{Co}_{3} \mathrm{~V}\right)$ & $-688\langle 2\rangle$ \\
\hline & & $\mathrm{Ir}_{2} \mathrm{Ta}\left(\mathrm{Ga}_{2} \mathrm{Hf}\right)$ & -659 \\
\hline & $\operatorname{IrTa}\left(\mathrm{L1}_{0} / \mathrm{IrTa}\right)$ & $\operatorname{IrTa}\left(\mathrm{L1}_{0}\right)$ & $-594\langle 3\rangle$ \\
\hline & $\operatorname{Ir}_{0.25} \operatorname{Ta}_{0.75}(\sigma)$ & $\operatorname{Ir}_{10} \operatorname{Ta}_{20}\left(\sigma_{A B B A B}\right)$ & -528 \\
\hline & & $\mathrm{IrTa}_{3}(\mathrm{~A} 15)$ & -479 \\
\hline \multirow[t]{4}{*}{ V } & $\mathrm{Ir}_{3} \mathrm{~V}\left(\mathrm{L1}_{2}\right)$ & $\mathrm{Ir}_{3} \mathrm{~V}\left(\mathrm{D} 0_{19}\right)$ & $-505\langle 21\rangle$ \\
\hline & $\operatorname{IrV}\left(\operatorname{IrV} / \mathrm{L1}_{0}\right)$ & $\operatorname{IrV}\left(\mathrm{L1}_{0}\right)$ & $-500^{\S}$ \\
\hline & $\operatorname{IrV}_{3}(\mathrm{~A} 15)$ & $\mathrm{IrV}_{3}(\mathrm{~A} 15)$ & -497 \\
\hline & & $\mathrm{IrV}_{8}\left(\mathrm{Pt}_{8} \mathrm{Ti}\right)$ & -225 \\
\hline
\end{tabular}

TABLE III. (Continued)

\begin{tabular}{|c|c|c|c|}
\hline \multicolumn{4}{|c|}{ Compounds } \\
\hline & Experiments $[35,36]$ & Calculations & $\Delta H \mathrm{meV} / \mathrm{at}$. \\
\hline \multirow[t]{4}{*}{ Mo } & $\mathrm{Ir}_{3} \mathrm{Mo}\left(\mathrm{D} 0_{19}\right)$ & $\mathrm{Ir}_{3} \mathrm{Mo}\left(\mathrm{D} 0_{19}\right)$ & -332 \\
\hline & & $\mathrm{Ir}_{2} \mathrm{Mo}(\mathrm{C} 37)$ & -337 \\
\hline & IrMo(B19) & $\mathrm{IrMo}(\mathrm{B} 19)$ & -321 \\
\hline & $\mathrm{IrMo}_{3}(\mathrm{~A} 15)$ & & $\langle 75\rangle$ \\
\hline \multirow[t]{4}{*}{ W } & & $\mathrm{Ir}_{8} \mathrm{~W}\left(\mathrm{Pt}_{8} \mathrm{Ti}\right)$ & -157 \\
\hline & $\mathrm{Ir}_{3} \mathrm{~W}\left(\mathrm{D} 0_{19}\right)$ & $\mathrm{Ir}_{3} \mathrm{~W}\left(\mathrm{DO}_{19}\right)$ & -350 \\
\hline & & $\mathrm{Ir}_{2} \mathrm{~W}(\mathrm{C} 37)$ & -352 \\
\hline & $\operatorname{IrW}(\mathrm{B} 19)$ & IrW(B19) & -300 \\
\hline \multirow[t]{4}{*}{$\mathrm{Cr}$} & $\mathrm{Cr}_{3} \operatorname{Ir}(\mathrm{A} 15)$ & & $\langle 48\rangle$ \\
\hline & $\mathrm{Cr}_{0.5} \operatorname{Ir}_{0.5}(\mathrm{Mg})$ & CrIr(B19) & -239 \\
\hline & & $\mathrm{CrIr}_{2}(\mathrm{C} 37)$ & -233 \\
\hline & & $\mathrm{CrIr}_{3}\left(\mathrm{D} 0_{19}\right)$ & -228 \\
\hline \multirow[t]{4}{*}{$\mathrm{Tc}$} & $\cdots$ & $\mathrm{Ir}_{8} \mathrm{Tc}\left(\mathrm{Pt}_{8} \mathrm{Ti}\right)$ & -89 \\
\hline & & $\mathrm{Ir}_{2} \mathrm{Tc}\left(\mathrm{Ir}_{2} \mathrm{Tc}^{\star}\right)$ & -224 \\
\hline & & $\operatorname{IrTc}(\mathrm{B} 19)$ & -287 \\
\hline & & $\mathrm{IrTc}_{3}\left(\mathrm{D} 0_{19}\right)$ & -217 \\
\hline \multirow[t]{4}{*}{$\operatorname{Re}$} & $\cdots$ & $\mathrm{Ir}_{8} \operatorname{Re}\left(\mathrm{Pt}_{8} \mathrm{Ti}\right)$ & -94 \\
\hline & & $\operatorname{Ir}_{2} \operatorname{Re}\left(\operatorname{Ir}_{2} \mathrm{Tc}^{\star}\right)$ & -227 \\
\hline & & $\operatorname{IrRe}(\mathrm{B} 19)$ & -274 \\
\hline & & $\operatorname{IrRe}_{3}\left(\mathrm{DO}_{19}\right)$ & -209 \\
\hline \multirow[t]{4}{*}{ Mn } & & $\mathrm{Ir}_{3} \mathrm{Mn}\left(\mathrm{L1}_{2}\right)$ & -173 \\
\hline & $\operatorname{IrMn}\left(\mathrm{L1}_{0}\right)$ & $\operatorname{IrMn}(\mathrm{B} 19)$ & $-204\langle 58\rangle$ \\
\hline & & $\operatorname{IrMn}_{2}(\mathrm{C} 37)$ & -175 \\
\hline & $\operatorname{IrMn}_{3}\left(\mathrm{L1}_{2}\right)$ & $\operatorname{IrMn}_{3}\left(\mathrm{L6}_{0}\right)$ & $-156\langle 108\rangle$ \\
\hline \multirow[t]{3}{*}{$\mathrm{Fe}$} & & $\mathrm{Fe}_{3} \operatorname{Ir}\left(\mathrm{LG}_{0}\right)$ & -44 \\
\hline & $\mathrm{Fe}_{0.6} \operatorname{Ir}_{0.4}(\mathrm{Mg})$ & FeIr(NbP) & -57 \\
\hline & & $\mathrm{FeIr}_{3}\left(\mathrm{DO}_{22}\right)$ & -63 \\
\hline \multirow[t]{2}{*}{ Os } & $\cdots$ & $\mathrm{Ir}_{8} \mathrm{Os}\left(\mathrm{Pt}_{8} \mathrm{Ti}\right)$ & -8 \\
\hline & & $\mathrm{IrOs}_{5}\left(\mathrm{Hf}_{5} \mathrm{Sc}^{\star}\right)$ & -7 \\
\hline \multirow[t]{6}{*}{$\mathrm{Ru}$} & $\ldots$ & $\mathrm{Ir}_{8} \mathrm{Ru}\left(\mathrm{Pt}_{8} \mathrm{Ti}\right)$ & -20 \\
\hline & & $\mathrm{Ir}_{3} \mathrm{Ru}\left(\mathrm{L1}_{2}\right)$ & -34 \\
\hline & & $\operatorname{IrRu}(\mathrm{B} 19)$ & -49 \\
\hline & & $\operatorname{IrRu}_{2}\left(\operatorname{Ir}_{2} \operatorname{Tc}^{\star}\right)$ & -54 \\
\hline & & $\mathrm{IrRu}_{3}\left(\mathrm{D} 0_{19}\right)$ & -53 \\
\hline & & $\mathrm{IrRu}_{5}\left(\mathrm{Hf}_{5} \mathrm{Sc}^{\star}\right)$ & -37 \\
\hline Co & $\cdots$ & $\ldots$ & \\
\hline $\mathrm{Ir}$ & Reference & & \\
\hline \multirow[t]{4}{*}{$\mathrm{Rh}$} & $\cdots$ & $\operatorname{Ir}_{3} \operatorname{Rh}\left(\mathrm{fcc}_{A B 3}^{[113]}\right)$ & -15 \\
\hline & & $\mathrm{Ir}_{2} \mathrm{Rh}\left(\mathrm{Pd}_{2} \mathrm{Ti}\right)$ & -20 \\
\hline & & $\operatorname{IrRh}\left(\operatorname{fcc}_{A 2 B 2}^{[113]}\right)$ & -21 \\
\hline & & $\mathrm{IrRh}_{2}\left(\mathrm{Pd}_{2} \mathrm{Ti}\right)$ & -18 \\
\hline $\mathrm{Ni}$ & $\cdots$ & $\mathrm{IrNi}(\mathrm{NbP})$ & -38 \\
\hline $\mathrm{Pt}$ & $\ldots$ & $\cdots$ & \\
\hline $\mathrm{Pd}$ & $\cdots$ & $\cdots$ & \\
\hline $\mathrm{Au}$ & $\ldots$ & $\ldots$ & \\
\hline
\end{tabular}


TABLE III. (Continued)

\begin{tabular}{|c|c|c|c|}
\hline \multicolumn{4}{|c|}{ Compounds } \\
\hline & Experiments $[35,36]$ & Calculations & $\Delta H \mathrm{meV} / \mathrm{at}$. \\
\hline $\mathrm{Ag}$ & $\cdots$ & $\cdots$ & \\
\hline $\mathrm{Cu}$ & $\ldots$ & $\ldots$ & \\
\hline $\mathrm{Hg}$ & $\ldots$ & $\ldots$ & \\
\hline $\mathrm{Cd}$ & $\ldots$ & $\ldots$ & \\
\hline \multirow[t]{4}{*}{$\mathrm{Zn}$} & & $\operatorname{IrZn}\left(\operatorname{IrZn}{ }^{\dagger}\right)$ & -195 \\
\hline & & $\mathrm{IrZn}_{2}(\mathrm{C} 49)$ & -238 \\
\hline & & $\operatorname{IrZn}_{3}\left(\mathrm{NbPd}_{3}\right)$ & -224 \\
\hline & $\mathrm{Ir}_{2} \mathrm{Zn}_{11}\left(\mathrm{Ir}_{2} \mathrm{Zn}_{11}\right)$ & $\mathrm{Ir}_{2} \mathrm{Zn}_{11}\left(\mathrm{Ir}_{2} \mathrm{Zn}_{11}\right)$ & -192 \\
\hline
\end{tabular}

TABLE IV. Compounds in Rhodium binary alloys. All symbols are as in Table III.

\begin{tabular}{|c|c|c|c|}
\hline \multicolumn{4}{|c|}{ Compounds } \\
\hline & Experiments $[35,36]$ & Calculations & $\Delta H \mathrm{meV} / \mathrm{at}$. \\
\hline \multirow[t]{7}{*}{$\mathrm{Y}$} & $\mathrm{Rh}_{3} \mathrm{Y}\left(\mathrm{CeNi}_{3}\right)$ & $\mathrm{Rh}_{3} \mathrm{Y}\left(\mathrm{CeNi}_{3}\right)$ & -569 \\
\hline & $\mathrm{Rh}_{2} \mathrm{Y}(\mathrm{C} 15)$ & $\mathrm{Rh}_{2} \mathrm{Y}(\mathrm{C} 15)$ & -742 \\
\hline & RhY(B2) & RhY(B2) & -863 \\
\hline & $\mathrm{Rh}_{2} \mathrm{Y}_{3}\left(\mathrm{Rh}_{2} \mathrm{Y}_{3}\right)$ & & $\langle 8\rangle$ \\
\hline & $\mathrm{Rh}_{3} \mathrm{Y}_{5}$ (unkn.) & $\mathrm{Rh}_{3} \mathrm{Y}_{5}\left(\mathrm{Pu}_{5} \mathrm{Rh}_{3}\right)$ & -727 \\
\hline & $\mathrm{Rh}_{3} \mathrm{Y}_{7}\left(\mathrm{Fe}_{3} \mathrm{Th}_{7}\right)$ & $\mathrm{Rh}_{3} \mathrm{Y}_{7}\left(\mathrm{Fe}_{3} \mathrm{Th}_{7}\right)$ & -606 \\
\hline & $\mathrm{RhY}_{3}\left(\mathrm{D} 0_{11}\right)$ & $\mathrm{RhY}_{3}\left(\mathrm{D} 0_{11}\right)$ & -517 \\
\hline \multirow[t]{6}{*}{$\mathrm{Sc}$} & & $\mathrm{Rh}_{7} \mathrm{Sc}\left(\mathrm{CuPt}_{7}\right)$ & -348 \\
\hline & $\mathrm{Rh}_{3} \mathrm{Sc}\left(\mathrm{L1}_{2}\right)$ & $\mathrm{Rh}_{3} \mathrm{Sc}\left(\mathrm{L1}_{2}\right)$ & -620 \\
\hline & $\operatorname{RhSc}(\mathrm{B} 2)$ & $\operatorname{RhSc}(\mathrm{B} 2)$ & -1035 \\
\hline & & $\mathrm{Ir}_{4} \mathrm{Sc}_{11}\left(\mathrm{Ir}_{4} \mathrm{Sc}_{11}\right)$ & -582 \\
\hline & $\mathrm{Rh}_{13} \mathrm{Sc}_{57}\left(\mathrm{Rh}_{13} \mathrm{Sc}_{57}\right)$ & $\mathrm{Rh}_{13} \mathrm{Sc}_{57}\left(\mathrm{Rh}_{13} \mathrm{Sc}_{57}\right)$ & -424 \\
\hline & & $\mathrm{Ir}_{7} \mathrm{Sc}_{44}\left(\mathrm{Mg}_{44} \mathrm{Rh}_{7}\right)$ & -319 \\
\hline \multirow[t]{6}{*}{$\mathrm{Zr}$} & $\mathrm{Rh}_{3} \mathrm{Zr}\left(\mathrm{L1}_{2}\right)$ & $\mathrm{Rh}_{3} \mathrm{Zr}\left(\mathrm{L1}_{2}\right)$ & -687 \\
\hline & $\mathrm{Rh}_{5} \mathrm{Zr}_{3}\left(\mathrm{Pd}_{5} \mathrm{Pu}_{3}\right)$ & $\mathrm{Rh}_{5} \mathrm{Zr}_{3}\left(\mathrm{Pd}_{5} \mathrm{Pu}_{3}\right)$ & -811 \\
\hline & $\mathrm{Rh}_{4} \mathrm{Zr}_{3}$ (unkn.) & $\mathrm{Rh}_{4} \mathrm{Zr}_{3}\left(\mathrm{Pd}_{4} \mathrm{Pu}_{3}\right)$ & -819 \\
\hline & RhZr(NiTi) & $\operatorname{RhZr(B33)}$ & $-790\langle 3\rangle$ \\
\hline & $\mathrm{RhZr}_{2}\left(\mathrm{NiTi}_{2} / \mathrm{C} 16\right)$ & $\mathrm{RhZr}_{2}\left(\mathrm{C} 11_{b}\right)$ & $-568\langle 34,11\rangle$ \\
\hline & $\mathrm{RhZr}_{3}\left(\mathrm{D} 0_{e}\right)$ & $\mathrm{IrZr}_{3}\left(\mathrm{SV}_{3}\right)$ & $-428^{\S}$ \\
\hline \multirow[t]{5}{*}{ Hf } & $\mathrm{Hf}_{2} \mathrm{Rh}\left(\mathrm{NiTi}_{2}\right)$ & $\mathrm{Hf}_{2} \mathrm{Rh}\left(\mathrm{CuZr}_{2}\right)$ & $-633\langle 13\rangle$ \\
\hline & $\operatorname{HfRh}(\mathrm{B} 2)$ & HfRh(B27) & $-898\langle 29\rangle$ \\
\hline & $\mathrm{Hf}_{3} \mathrm{Rh}_{4}$ (unkn.) & & \\
\hline & $\mathrm{Hf}_{3} \mathrm{Rh}_{5}\left(\mathrm{Ge}_{3} \mathrm{Rh}_{5}\right)$ & $\mathrm{Hf}_{3} \mathrm{Rh}_{5}\left(\mathrm{Ge}_{3} \mathrm{Rh}_{5}\right)$ & -928 \\
\hline & $\mathrm{HfRh}_{3}\left(\mathrm{L1}_{2}\right)$ & $\operatorname{HfRh}_{3}\left(\mathrm{L1}_{2}\right)$ & -762 \\
\hline \multirow[t]{6}{*}{$\mathrm{Ti}$} & & $\mathrm{Rh}_{7} \mathrm{Ti}\left(\mathrm{CuPt}_{7}\right)$ & -330 \\
\hline & $\mathrm{Rh}_{5} \mathrm{Ti}$ (unkn.) & & \\
\hline & $\mathrm{Rh}_{3} \operatorname{Ti}\left(\mathrm{L1}_{2}\right)$ & $\mathrm{Rh}_{3} \mathrm{Ti}\left(\mathrm{Ll}_{2}\right)$ & -631 \\
\hline & $\mathrm{Rh}_{5} \mathrm{Ti}_{3}\left(\mathrm{Ge}_{3} \mathrm{Rh}_{5}\right)$ & $\mathrm{Rh}_{5} \mathrm{Ti}_{3}\left(\mathrm{Ge}_{3} \mathrm{Rh}_{5}\right)$ & -790 \\
\hline & RhTi (unkn.) & $\operatorname{RhTi}\left(\mathrm{L1}_{0}\right)$ & -749 \\
\hline & $\mathrm{RhTi}_{2}\left(\mathrm{CuZr}_{2}\right)$ & $\mathrm{RhTi}_{2}\left(\mathrm{C} 11_{b}\right)$ & $-629\langle 1\rangle$ \\
\hline
\end{tabular}

TABLE IV. (Continued)

\begin{tabular}{|c|c|c|c|}
\hline \multicolumn{4}{|c|}{ Compounds } \\
\hline & Experiments $[35,36]$ & Calculations & $\Delta H$ meV/at. \\
\hline \multirow[t]{5}{*}{$\mathrm{Nb}$} & & $\mathrm{Nb}_{8} \mathrm{Rh}\left(\mathrm{Pt}_{8} \mathrm{Ti}\right)$ & -131 \\
\hline & $\mathrm{Nb}_{3} \mathrm{Rh}(\mathrm{A} 15)$ & $\mathrm{Nb}_{3} \mathrm{Rh}(\mathrm{A} 15)$ & -288 \\
\hline & $\mathrm{Nb}_{0.7} \mathrm{Rh}_{0.3}(\sigma)$ & $\mathrm{Nb}_{20} \mathrm{Rh}_{10}\left(\sigma_{B A A B A}\right)$ & -342 \\
\hline & $\operatorname{NbRh}\left(\mathrm{L1}_{0} / \mathrm{IrTa}\right)$ & $\operatorname{NbRh}\left(\mathrm{L1}_{0}\right)$ & $-436\langle 4\rangle$ \\
\hline & $\mathrm{NbRh}_{3}\left(\mathrm{Ll}_{2} / \mathrm{Co}_{3} \mathrm{~V}\right)$ & $\mathrm{NbRh}_{3}\left(\mathrm{Co}_{3} \mathrm{~V}\right)$ & $-548\langle 6\rangle$ \\
\hline \multirow[t]{6}{*}{$\mathrm{Ta}$} & $\mathrm{Rh}_{3} \mathrm{Ta}\left(\mathrm{L1}_{2}\right)$ & $\mathrm{Rh}_{3} \mathrm{Ta}\left(\mathrm{L1}_{2}\right)$ & -611 \\
\hline & $\mathrm{Rh}_{2} \mathrm{Ta}(\mathrm{C} 37)$ & $\mathrm{Rh}_{2} \mathrm{Ta}\left(\mathrm{Ga}_{2} \mathrm{Hf}\right)$ & $-597\langle 13\rangle$ \\
\hline & RhTa(IrTa) & & $\langle 11\rangle$ \\
\hline & $\mathrm{Rh}_{0.3} \mathrm{Ta}_{0.7}(\sigma)$ & $\mathrm{RhTa}_{3}(\mathrm{~A} 15)$ & -333 \\
\hline & & $\operatorname{RhTa}_{5}\left(\operatorname{RuTc}_{5}^{\star}\right)$ & -233 \\
\hline & & $\mathrm{RhTa}_{8}\left(\mathrm{Pt}_{8} \mathrm{Ti}\right)$ & -159 \\
\hline \multirow[t]{6}{*}{ V } & & $\mathrm{Rh}_{5} \mathrm{~V}\left(\mathrm{HfPd}_{5}^{\star}\right)$ & -268 \\
\hline & $\mathrm{Rh}_{3} \mathrm{~V}\left(\mathrm{~L} 1_{2}\right)$ & $\mathrm{Rh}_{3} \mathrm{~V}\left(\mathrm{D} 0_{19}\right)$ & $-393\langle 11\rangle$ \\
\hline & $\operatorname{RhV}\left(\operatorname{IrV} / \mathrm{L1}_{0}\right)$ & $\operatorname{RhV}\left(\mathrm{L1}_{0}\right)$ & $-371^{\S}$ \\
\hline & $\mathrm{RhV}_{3}(\mathrm{~A} 15)$ & $\mathrm{RhV}_{3}(\mathrm{~A} 15)$ & -332 \\
\hline & & $\operatorname{RhV}_{5}\left(\operatorname{RuTc}_{5}^{\star}\right)$ & -246 \\
\hline & & $\mathrm{RhV}_{8}\left(\mathrm{Pt}_{8} \mathrm{Ti}\right)$ & -170 \\
\hline \multirow[t]{4}{*}{ Mo } & $\operatorname{MoRh}(\mathrm{B} 19)$ & $\operatorname{MoRh}(\mathrm{B} 19)$ & -196 \\
\hline & & $\mathrm{MoRh}_{2}(\mathrm{C} 37)$ & -247 \\
\hline & $\mathrm{MoRh}_{3}\left(\mathrm{D0}_{19}\right)$ & $\mathrm{MoRh}_{3}\left(\mathrm{D} 0_{19}\right)$ & -248 \\
\hline & & $\mathrm{MoRh}_{8}\left(\mathrm{Pt}_{8} \mathrm{Ti}\right)$ & -116 \\
\hline \multirow[t]{3}{*}{$\mathrm{W}$} & $\mathrm{Rh}_{0.8} \mathrm{~W}_{0.2}(\mathrm{Mg})$ & $\mathrm{Rh}_{8} \mathrm{~W}\left(\mathrm{Pt}_{8} \mathrm{Ti}\right)$ & -140 \\
\hline & $\mathrm{Rh}_{3} \mathrm{~W}\left(\mathrm{D} 0_{19}\right)$ & $\mathrm{Rh}_{3} \mathrm{~W}\left(\mathrm{D} 0_{19}\right)$ & -274 \\
\hline & & $\mathrm{Rh}_{2} \mathrm{~W}(\mathrm{C} 37)$ & -264 \\
\hline \multirow[t]{4}{*}{$\mathrm{Cr}$} & $\mathrm{Cr}_{3} \mathrm{Rh}(\mathrm{A} 15)$ & & $\langle 103\rangle$ \\
\hline & & $\mathrm{CrRh}_{2}(\mathrm{C} 37)$ & -117 \\
\hline & $\mathrm{CrRh}_{3}\left(\mathrm{L1}_{2}\right)$ & $\mathrm{CrRh}_{3}\left(\mathrm{L1}_{2}\right)$ & -128 \\
\hline & & $\mathrm{CrRh}_{7}\left(\mathrm{CuPt}_{7}\right)$ & -65 \\
\hline \multirow[t]{3}{*}{$\mathrm{Tc}$} & $\cdots$ & $\mathrm{Rh}_{2} \mathrm{Tc}\left(\mathrm{Ir}_{2} \mathrm{Tc}^{\star}\right)$ & -157 \\
\hline & & RhTc(B19) & -175 \\
\hline & & $\mathrm{RhTc}_{3}\left(\mathrm{D} 0_{19}\right)$ & -158 \\
\hline \multirow[t]{3}{*}{$\operatorname{Re}$} & $\cdots$ & $\operatorname{Re}_{3} \operatorname{Rh}\left(\mathrm{DO}_{19}\right)$ & -163 \\
\hline & & $\operatorname{ReRh}(\mathrm{B} 19)$ & -184 \\
\hline & & $\operatorname{ReRh}_{2}\left(\operatorname{Ir}_{2} \mathrm{Tc}^{\star}\right)$ & -173 \\
\hline \multirow[t]{4}{*}{ Mn } & $\mathrm{Mn}_{3} \operatorname{Rh}\left(\mathrm{L1}_{2}\right)$ & & $\langle 153\rangle$ \\
\hline & $\operatorname{MnRh}(\mathrm{B} 2)$ & $\operatorname{MnRh}(\mathrm{B} 2)$ & -190 \\
\hline & & $\mathrm{MnRh}_{3}\left(\mathrm{L1}_{2}\right)$ & -126 \\
\hline & & $\mathrm{MnRh}_{7}\left(\mathrm{CuPt}_{7}\right)$ & -66 \\
\hline \multirow[t]{4}{*}{$\mathrm{Fe}$} & & $\mathrm{Fe}_{3} \mathrm{Rh}\left(\mathrm{bcc}_{A B 3}^{[001]}\right)$ & -49 \\
\hline & & $\mathrm{Fe}_{2} \mathrm{Rh}\left(\mathrm{Fe}_{2} \mathrm{Rh}^{\dagger}\right)$ & -57 \\
\hline & $\mathrm{FeRh}(\mathrm{B} 2)$ & & $\langle 1\rangle$ \\
\hline & & $\mathrm{FeRh}_{3}\left(\mathrm{DO}_{24}\right)$ & -56 \\
\hline Os & $\cdots$ & $\operatorname{OsRh}\left(\mathrm{RhRu}^{\star}\right)$ & -8 \\
\hline \multirow[t]{3}{*}{$\mathrm{Ru}$} & $\cdots$ & $\mathrm{Rh}_{8} \mathrm{Ru}\left(\mathrm{Pt}_{8} \mathrm{Ti}\right)$ & -2 \\
\hline & & $\mathrm{RhRu}\left(\mathrm{RhRu}^{\star}\right)$ & -8 \\
\hline & & $\mathrm{RhRu}_{2}\left(\mathrm{RhRu}_{2}{ }^{\star}\right)$ & -6 \\
\hline
\end{tabular}


TABLE IV. (Continued)

\begin{tabular}{|c|c|c|c|}
\hline \multicolumn{4}{|c|}{ Compounds } \\
\hline & Experiments $[35,36]$ & Calculations & $\Delta H \mathrm{meV} / \mathrm{at}$. \\
\hline & & $\mathrm{RhRu}_{5}\left(\mathrm{RhRu}_{5}{ }^{\star}\right)$ & -3 \\
\hline $\mathrm{Co}$ & $\cdots$ & $\ldots$ & \\
\hline Ir & $\cdots$ & $\begin{array}{c}\operatorname{Ir}_{3} \operatorname{Rh}\left(\operatorname{fcc}_{A B 3}^{[113]}\right) \\
\operatorname{Ir}_{2} \operatorname{Rh}\left(\mathrm{Pd}_{2} \mathrm{Ti}\right) \\
\operatorname{IrRh}\left(\mathrm{fcc}_{A 2 B 2}^{[113]}\right) \\
\operatorname{IrRh}_{2}\left(\mathrm{Pd}_{2} \mathrm{Ti}\right) \\
\end{array}$ & $\begin{array}{l}-15 \\
-20 \\
-21 \\
-18\end{array}$ \\
\hline $\mathrm{Rh}$ & Reference & & \\
\hline $\mathrm{Ni}$ & $\cdots$ & $\cdots$ & \\
\hline $\mathrm{Pt}$ & $\cdots$ & $\begin{array}{c}\mathrm{PtRh}(\mathrm{NbP}) \\
\mathrm{PtRh}_{2}\left(\mathrm{Pd}_{2} \mathrm{Ti}\right) \\
\mathrm{PtRh}_{3}\left(\mathrm{DO}_{22}\right) \\
\end{array}$ & $\begin{array}{l}-25 \\
-21 \\
-18 \\
\end{array}$ \\
\hline $\mathrm{Pd}$ & $\cdots$ & $\cdots$ & \\
\hline $\mathrm{Au}$ & $\cdots$ & $\cdots$ & \\
\hline $\mathrm{Ag}$ & $\cdots$ & $\cdots$ & \\
\hline $\mathrm{Cu}$ & $\cdots$ & $\mathrm{Cu}_{7} \mathrm{Rh}\left(\mathrm{CuPt}_{7}\right)$ & -4 \\
\hline $\mathrm{Hg}$ & $\begin{array}{c}\text { " } \mathrm{Hg}_{5} \mathrm{Rh} " \\
\text { " } \mathrm{Hg}_{4.63} \mathrm{Rh}, \\
\mathrm{Hg}_{2} \mathrm{Rh}\left(\mathrm{Hg}_{2} \mathrm{Pt}\right)\end{array}$ & $\mathrm{Hg}_{4} \mathrm{Rh}\left(\mathrm{Hg}_{4} \mathrm{Pt}\right)$ & $\begin{array}{l}-40 \\
\langle 28\rangle\end{array}$ \\
\hline $\mathrm{Cd}$ & $\begin{array}{c}\text { "Cd }{ }_{21} \mathrm{Rh}_{5} " \\
(\gamma \text {-brass })\end{array}$ & $\begin{array}{l}\mathrm{Cd}_{4} \mathrm{Rh}\left(\mathrm{Hg}_{4} \mathrm{Pt}\right) \\
\mathrm{Cd}_{2} \mathrm{Rh}\left(\mathrm{Hg}_{2} \mathrm{Pt}\right) \\
\end{array}$ & $\begin{array}{l}-104 \\
-166 \\
\end{array}$ \\
\hline $\mathrm{Zn}$ & $\begin{array}{c}\text { " } \mathrm{Rh}_{5} \mathrm{Zn}_{21} " \\
(\gamma \text {-brass })\end{array}$ & $\begin{array}{c}\mathrm{RhZn}(\mathrm{B} 2) \\
\mathrm{Rh}_{3} \mathrm{Zn}_{5}\left(\mathrm{Ga}_{3} \mathrm{Pt}_{5}\right) \\
\mathrm{RhZn}_{2}\left(\mathrm{ZrSi}_{2}\right) \\
\mathrm{RhZn}_{3}\left(\mathrm{D} 0_{23}\right) \\
\mathrm{Rh}_{2} \mathrm{Zn}_{11}(\mathrm{ICSD} \# 107576) \\
\mathrm{RhZn}_{13}(\mathrm{ICSD \# 107575)}\end{array}$ & $\begin{array}{l}-391 \\
-395 \\
-388 \\
-351 \\
-250 \\
-129\end{array}$ \\
\hline
\end{tabular}

forming when reaching thermodynamic equilibrium is presumably easier. That is not to say, however, that the predicted phases will necessarily be difficult to synthesize-some of the systems where the $T_{s}$ value is small have been experimentally observed to be compound forming (e.g., Cr-Pd, Au-Pd, Ag-Pt, Hg-Rh, and Co-Pt). $T_{s}$ decreases gradually as we move from the centers of the compound-forming clusters towards their edges. Most systems with low $T_{s}$ are adjacent to the remaining noncompound-forming region. This leads to a qualitative picture of compound stability against disorder, which is correlated with the position of a system within the compound-forming cluster and with larger clusters centered at systems with more stable structures.

It is instructive to note that many obscure and large unit cell structures that are reported in the experimental literature are recovered in the HT search. For example, compounds of prototypes such as $\mathrm{Mg}_{44} \mathrm{Rh}_{7}, \mathrm{Ru}_{25} \mathrm{Y}_{44}, \mathrm{Ir}_{4} \mathrm{Sc}_{11}$,
TABLE V. Compounds in Platinum binary alloys. The term tet- $\mathrm{L} 1_{2}$ denotes a tetragonal distortion of the $\mathrm{L} 1_{2}$ structure. All symbols are as in Table III.

\begin{tabular}{|c|c|c|c|}
\hline \multicolumn{4}{|c|}{ Compounds } \\
\hline & Experiments $[35,36]$ & Calculations & $\Delta H \mathrm{meV} / \mathrm{at}$. \\
\hline \multirow[t]{10}{*}{$\mathrm{Y}$} & $\mathrm{Pt}_{5} \mathrm{Y}$ (unkn.) & $\mathrm{Pt}_{5} \mathrm{Y}\left(\mathrm{D} 2_{d}\right)$ & -677 \\
\hline & $\mathrm{Pt}_{3} \mathrm{Y}\left(\mathrm{L1}_{2}\right)$ & $\mathrm{Pt}_{3} \mathrm{Y}\left(\mathrm{L1}_{2}\right)$ & -983 \\
\hline & $\mathrm{Pt}_{2} \mathrm{Y}(\mathrm{C} 15)$ & $\mathrm{Pt}_{2} \mathrm{Y}(\mathrm{C} 15)$ & -1095 \\
\hline & $\mathrm{Pt}_{4} \mathrm{Y}_{3}$ (unkn.) & $\mathrm{Pt}_{4} \mathrm{Y}_{3}\left(\mathrm{Pd}_{4} \mathrm{Pu}_{3}\right)$ & -1208 \\
\hline & PtY(B27) & PtY(B33) & $-1252\langle 54\rangle$ \\
\hline & $\mathrm{Pt}_{4} \mathrm{Y}_{5}\left(\mathrm{Ge}_{4} \mathrm{Sm}_{5}\right)$ & & $\langle 1\rangle$ \\
\hline & $\mathrm{Pt}_{3} \mathrm{Y}_{5}\left(\mathrm{Mn}_{5} \mathrm{Si}_{3}\right)$ & & $\langle 28\rangle$ \\
\hline & $\mathrm{PtY}_{2}\left(\mathrm{Cl}_{2} \mathrm{~Pb}\right)$ & $\mathrm{PtY}_{2}\left(\mathrm{Cl}_{2} \mathrm{~Pb}\right)$ & -936 \\
\hline & $\mathrm{Pt}_{3} \mathrm{Y}_{7}\left(\mathrm{Fe}_{3} \mathrm{Th}_{7}\right)$ & & $\langle 19\rangle$ \\
\hline & $\mathrm{PtY}_{3}\left(\mathrm{D} 0_{11}\right)$ & $\mathrm{PtY}_{3}\left(\mathrm{D} 0_{11}\right)$ & -709 \\
\hline \multirow[t]{7}{*}{$\mathrm{Sc}$} & & $\mathrm{Pt}_{8} \mathrm{Sc}\left(\mathrm{Pt}_{8} \mathrm{Ti}\right)$ & -482 \\
\hline & $\mathrm{Pt}_{3} \mathrm{Sc}\left(\mathrm{L1}_{2}\right)$ & $\mathrm{Pt}_{3} \mathrm{Sc}\left(\mathrm{L1}_{2}\right)$ & -1050 \\
\hline & & $\mathrm{Pt}_{2} \mathrm{Sc}\left(\mathrm{Ga}_{2} \mathrm{Hf}\right)$ & -1143 \\
\hline & & $\mathrm{Pt}_{4} \mathrm{Sc}_{3}\left(\mathrm{Pd}_{4} \mathrm{Pu}_{3}\right)$ & -1197 \\
\hline & $\mathrm{PtSc}(\mathrm{B} 2)$ & $\operatorname{PtSc}(\mathrm{B} 2)$ & -1232 \\
\hline & $\mathrm{PtSc}_{2}\left(\mathrm{Cl}_{2} \mathrm{~Pb}\right)$ & $\mathrm{PtSc}_{2}\left(\mathrm{Cl}_{2} \mathrm{~Pb}\right)$ & -982 \\
\hline & $\mathrm{Pt}_{13} \mathrm{Sc}_{57}\left(\mathrm{Rh}_{13} \mathrm{Sc}_{57}\right)$ & $\mathrm{Pt}_{13} \mathrm{Sc}_{57}\left(\mathrm{Rh}_{13} \mathrm{Sc}_{57}\right)$ & -571 \\
\hline \multirow[t]{7}{*}{$\mathrm{Zr}$} & $\mathrm{Pt}_{8} \mathrm{Zr}\left(\mathrm{Pt}_{8} \mathrm{Ti}\right)$ & $\mathrm{Pt}_{8} \mathrm{Zr}\left(\mathrm{Pt}_{8} \mathrm{Ti}\right)$ & -496 \\
\hline & $\mathrm{Pt}_{3} \mathrm{Zr}\left(\mathrm{D} 0_{24} / \mathrm{L1}_{2}\right)$ & $\mathrm{Pt}_{3} \mathrm{Zr}\left(\mathrm{DO}_{24}\right)$ & $-1031\langle 12\rangle$ \\
\hline & $\mathrm{Pt}_{2} \mathrm{Zr}\left(\mathrm{C} 11_{b}\right)$ & & $\langle 62\rangle$ \\
\hline & $\mathrm{Pt}_{11} \mathrm{Zr}_{9}\left(\mathrm{Pt}_{11} \mathrm{Zr}_{9}\right)$ & & $\langle 73\rangle$ \\
\hline & $\operatorname{PtZr}(\mathrm{TlI})$ & PtZr(B33) & $-1087\langle 1\rangle$ \\
\hline & $\mathrm{Pt}_{3} \mathrm{Zr}_{5}\left(\mathrm{D} 8_{8}\right)$ & & $\langle 25\rangle$ \\
\hline & $\mathrm{PtZr}_{2}\left(\mathrm{NiTi}_{2}\right)$ & $\mathrm{PtZr}_{2}(\mathrm{C} 16)$ & $-759\langle 51\rangle$ \\
\hline \multirow[t]{4}{*}{$\mathrm{Hf}$} & $\mathrm{Hf}_{2} \mathrm{Pt}\left(\mathrm{NiTi}_{2}\right)$ & $\mathrm{Hf}_{2} \mathrm{Pt}\left(\mathrm{NiTi}_{2}\right)$ & -786 \\
\hline & $\mathrm{HfPt}(\mathrm{B} 2 / \mathrm{B} 33 / \mathrm{TlI})$ & $\operatorname{HfPt}(\mathrm{B} 33 / \mathrm{TII})$ & $-1155\langle 165\rangle$ \\
\hline & $\mathrm{HfPt}_{3}\left(\mathrm{L1}_{2} / \mathrm{D0}_{24}\right)$ & $\mathrm{HfPt}_{3}\left(\mathrm{DO}_{24}\right)$ & $-1100\langle 3\rangle$ \\
\hline & & $\mathrm{HfPt}_{8}\left(\mathrm{Pt}_{8} \mathrm{Ti}\right)$ & -528 \\
\hline \multirow[t]{6}{*}{$\mathrm{Ti}$} & $\mathrm{Pt}_{8} \mathrm{Ti}\left(\mathrm{Pt}_{8} \mathrm{Ti}\right)$ & $\mathrm{Pt}_{8} \mathrm{Ti}\left(\mathrm{Pt}_{8} \mathrm{Ti}\right)$ & -433 \\
\hline & & $\mathrm{Pt}_{5} \mathrm{Ti}\left(\mathrm{HfPd}_{5}^{\star}\right)$ & -617 \\
\hline & $\mathrm{Pt}_{3} \mathrm{Ti}\left(\mathrm{D} 0_{24} / \mathrm{L1}_{2}\right)$ & $\mathrm{Pt}_{3} \mathrm{Ti}\left(\mathrm{PuAl}_{3}\right)$ & $-864\langle 3,5\rangle$ \\
\hline & $\mathrm{Pt}_{5} \mathrm{Ti}_{3}\left(\mathrm{Au}_{5} \mathrm{GaZn}_{2}\right)$ & $\mathrm{Pt}_{5} \mathrm{Ti}_{3}\left(\mathrm{Au}_{5} \mathrm{GaZn}_{2}\right)$ & -952 \\
\hline & $\operatorname{PtTi}(\mathrm{B} 19)$ & PtTi(NiTi) & $-933\langle 5\rangle$ \\
\hline & $\mathrm{PtTi}_{3}(\mathrm{~A} 15)$ & $\mathrm{PtTi}_{3}(\mathrm{~A} 15)$ & -648 \\
\hline \multirow[t]{6}{*}{$\mathrm{Nb}$} & $\mathrm{Nb}_{3} \mathrm{Pt}(\mathrm{A} 15)$ & $\mathrm{Nb}_{3} \mathrm{Pt}(\mathrm{A} 15)$ & -415 \\
\hline & $\mathrm{Nb}_{0.6} \mathrm{Pt}_{0.4}(\sigma)$ & & \\
\hline & $\operatorname{NbPt}(\mathrm{B} 19)$ & $\operatorname{NbPt}\left(\mathrm{L1}_{0}\right)$ & $-660\langle 13\rangle$ \\
\hline & $\mathrm{NbPt}_{2}\left(\mathrm{MoPt}_{2}\right)$ & $\mathrm{NbPt}_{2}\left(\mathrm{MoPt}_{2}\right)$ & -721 \\
\hline & $\mathrm{NbPt}_{3}\left(\mathrm{L6}_{0} / \mathrm{NbPt}_{3}\right)$ & $\mathrm{NbPt}_{3}\left(\mathrm{NbPt}_{3} / \mathrm{DO}_{a}\right)$ & $-678\langle 154\rangle$ \\
\hline & & $\mathrm{NbPt}_{8}\left(\mathrm{Pt}_{8} \mathrm{Ti}\right)$ & -378 \\
\hline \multirow[t]{7}{*}{$\mathrm{Ta}$} & & $\mathrm{Pt}_{8} \mathrm{Ta}\left(\mathrm{Pt}_{8} \mathrm{Ti}\right)$ & -416 \\
\hline & $\begin{array}{c}\mathrm{Pt}_{3} \mathrm{Ta}\left(\mathrm{DO}_{22} / \mathrm{L}_{0} /\right. \\
\left.\mathrm{NbPt}_{3}\right)\end{array}$ & $\mathrm{Pt}_{3} \mathrm{Ta}\left(\mathrm{NbPt}_{3}\right)$ & $-723\langle 11,183\rangle$ \\
\hline & $\mathrm{Pt}_{2} \mathrm{Ta}\left(\mathrm{Au}_{2} \mathrm{~V}\right)$ & $\mathrm{Pt}_{2} \mathrm{Ta}\left(\mathrm{Au}_{2} \mathrm{~V}\right)$ & -757 \\
\hline & & $\operatorname{PtTa}\left(\mathrm{L} 1_{0}\right)$ & -643 \\
\hline & $\mathrm{Pt}_{0.25} \mathrm{Ta}_{0.75}(\sigma)$ & $\mathrm{Pt}_{8} \mathrm{Ta}_{22}\left(\sigma_{B B B A B}\right)$ & -434 \\
\hline & $\mathrm{Pt}_{0.6} \mathrm{Ta}_{3.74}(\mathrm{~A} 15)$ & $\mathrm{PtTa}_{3}(\mathrm{~A} 15)$ & -416 \\
\hline & & $\mathrm{PtTa}_{8}\left(\mathrm{Pt}_{8} \mathrm{Ti}\right)$ & -197 \\
\hline
\end{tabular}


TABLE V. (Continued)

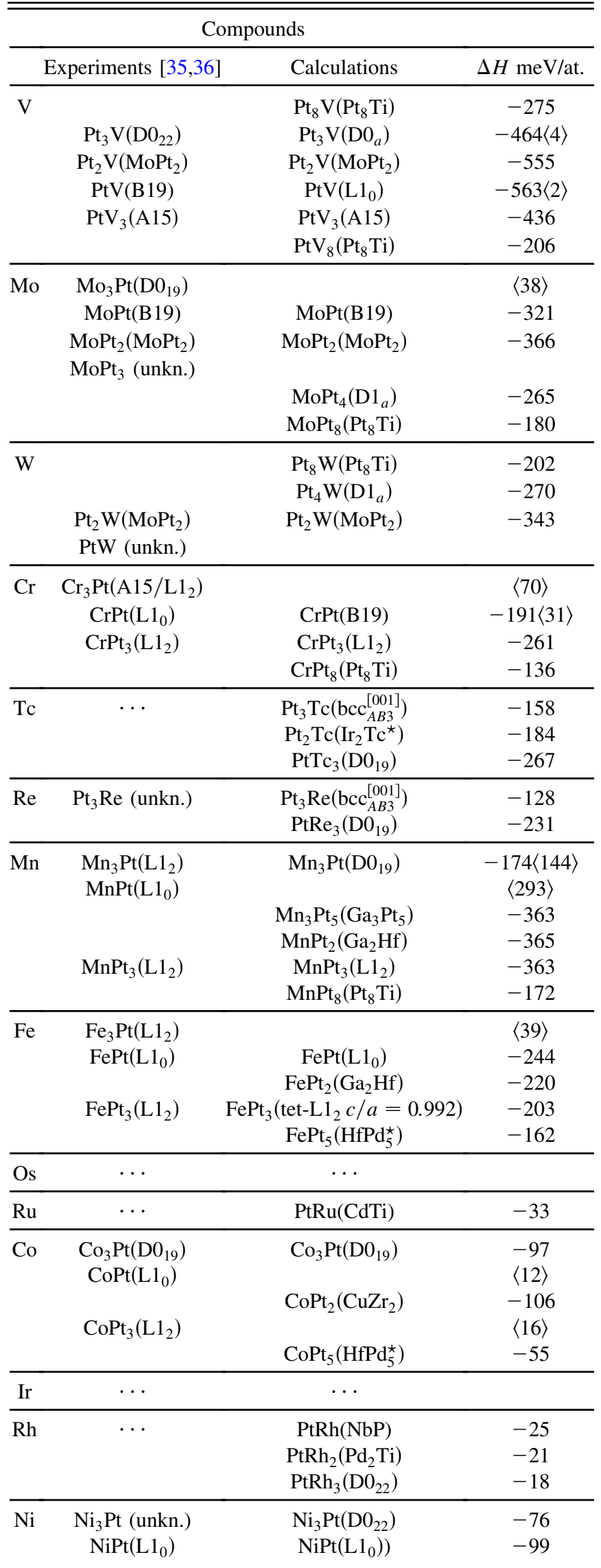

TABLE V. (Continued)

\begin{tabular}{|c|c|c|c|}
\hline \multicolumn{4}{|c|}{ Compounds } \\
\hline & Experiments $[35,36]$ & Calculations & $\Delta H \mathrm{meV} / \mathrm{at}$. \\
\hline & & $\begin{array}{c}\mathrm{NiPt}_{2}\left(\mathrm{CuZr}_{2}\right) \\
\mathrm{NiPt}_{3}\left(\mathrm{DO}_{23}\right)\end{array}$ & $\begin{array}{l}-75 \\
-61 \\
\end{array}$ \\
\hline $\mathrm{Pt}$ & Reference & & \\
\hline $\mathrm{Pd}$ & $\cdots$ & $\begin{array}{c}\mathrm{Pd}_{7} \mathrm{Pt}\left(\mathrm{CuPt}_{7}\right) \\
\operatorname{Pd}_{3} \operatorname{Pt}\left(\mathrm{CdPt}_{3}^{\star}\right) \\
\operatorname{PdPt}\left(\mathrm{L1}_{1}\right) \\
\operatorname{PdPt}_{3}\left(\mathrm{L1}_{2}\right) \\
\operatorname{PdPt}_{7}\left(\mathrm{CuPt}_{7}\right) \\
\end{array}$ & $\begin{array}{l}-14 \\
-25 \\
-36 \\
-26 \\
-15\end{array}$ \\
\hline $\mathrm{Au}$ & $\cdots$ & $\cdots$ & \\
\hline $\mathrm{Ag}$ & $\begin{array}{c}\mathrm{Ag}_{3} \mathrm{Pt}\left(\mathrm{Ll}_{2}\right) \\
\mathrm{AgPt} \text { (unkn.) } \\
\mathrm{AgPt}_{3} \text { (unkn.) }\end{array}$ & $\begin{array}{c}\mathrm{Ag}_{7} \mathrm{Pt}\left(\mathrm{CuPt}_{7}\right) \\
\mathrm{Ag}_{3} \mathrm{Pt}_{2}\left(\mathrm{Ag}_{3} \mathrm{Pt}_{2}^{\dagger}\right) \\
\operatorname{AgPt}\left(\mathrm{Ll}_{1}\right)\end{array}$ & $\begin{array}{l}-13 \\
\langle 34\rangle \\
-38 \\
-39\end{array}$ \\
\hline $\mathrm{Cu}$ & $\begin{array}{c}\mathrm{Cu}_{7} \mathrm{Pt}\left(\mathrm{CuPt}_{7}\right) \\
\mathrm{Cu}_{3} \mathrm{Pt}\left(\mathrm{L1}_{2}\right) \\
\mathrm{CuPt}\left(\mathrm{L1}_{1}\right) \\
\mathrm{Cu}_{3} \mathrm{Pt}_{5} \text { (unkn.) } \\
\mathrm{CuPt}_{3} \text { (unkn.) } \\
\mathrm{CuPt}_{7}\left(\mathrm{CuPt}_{7}\right)\end{array}$ & $\begin{array}{c}\mathrm{Cu}_{7} \mathrm{Pt}\left(\mathrm{CuPt}_{7}\right) \\
\mathrm{Cu}_{3} \mathrm{Pt}\left(\mathrm{L1}_{2}\right) \\
\mathrm{CuPt}\left(\mathrm{L1}_{1}\right)\end{array}$ & $\begin{array}{l}-87 \\
-143 \\
-166\end{array}$ \\
\hline $\mathrm{Hg}$ & $\begin{array}{l}\mathrm{Hg}_{4} \mathrm{Pt}\left(\mathrm{Hg}_{4} \mathrm{Pt}\right) \\
\mathrm{Hg}_{2} \mathrm{Pt}\left(\mathrm{Hg}_{2} \mathrm{Pt}\right) \\
\mathrm{HgPt}_{3} \text { (unkn.) }\end{array}$ & $\mathrm{Hg}_{4} \mathrm{Pt}\left(\mathrm{Hg}_{4} \mathrm{Pt}\right)$ & $\begin{array}{l}-104 \\
\langle 25\rangle\end{array}$ \\
\hline $\mathrm{Cd}$ & $\begin{array}{c}\mathrm{Cd}_{5} \mathrm{Pt}\left(\mathrm{Cd}_{5} \mathrm{Pt}-\right. \\
\text { partial occupancy })\end{array}$ & & \\
\hline & $\begin{array}{c}\mathrm{Cd}_{3} \mathrm{Pt} \text { (unkn.) } \\
\mathrm{Cd}_{7} \mathrm{Pt}_{3} \text { (unkn.) } \\
\mathrm{Cd}_{2} \mathrm{Pt} \text { (unkn.) } \\
\mathrm{CdPt}\left(\mathrm{L1}_{0}\right) \\
\mathrm{CdPt}_{3}\left(\mathrm{L1}_{2}\right)\end{array}$ & $\begin{array}{c}\mathrm{Cd}_{2} \mathrm{Pt}\left(\mathrm{Hg}_{2} \mathrm{Pt}\right) \\
\mathrm{CdPt}\left(\mathrm{Ll}_{0}\right) \\
\mathrm{CdPt}_{3}\left(\mathrm{CdPt}_{3}^{\star}\right) \\
\mathrm{CdPt}_{7}\left(\mathrm{CuPt}_{7}\right)\end{array}$ & $\begin{array}{l}-228 \\
-260 \\
\\
-316 \\
-322 \\
-190\langle 11\rangle \\
-114 \\
\end{array}$ \\
\hline $\mathrm{Zn}$ & $\begin{array}{c}\mathrm{Pt}_{3} \mathrm{Zn}\left(\mathrm{L1}_{2}\right) \\
\mathrm{PtZn}\left(\mathrm{L1}_{0}\right) \\
\mathrm{Pt}_{7} \mathrm{Zn}_{12}\left(\mathrm{Pt}_{7} \mathrm{Zn}_{12}\right) \\
\mathrm{PtZn}_{2} \text { (unkn.) }\end{array}$ & $\begin{array}{c}\mathrm{Pt}_{7} \mathrm{Hg}\left(\mathrm{CuPt}_{7}\right) \\
\mathrm{Pt}_{3} \mathrm{Zn}\left(\mathrm{CdPt}_{3}^{\star}\right) \\
\mathrm{PtZn}\left(\mathrm{L1}_{0}\right) \\
\mathrm{Pt}_{7} \mathrm{Zn}_{12}\left(\mathrm{Pt}_{7} \mathrm{Zn}_{12}\right) \\
\mathrm{PtZn}_{2}(\mathrm{C} 49) \\
\mathrm{PtZn}_{3}\left(\mathrm{DO}_{22}\right) \\
\mathrm{Pt}_{2} \mathrm{Zn}_{11}\left(\mathrm{Ir}_{2} \mathrm{Zn}_{11}\right)\end{array}$ & $\begin{array}{l}-189 \\
-331\langle 6\rangle \\
-570 \\
-489 \\
-463 \\
-397 \\
-272\end{array}$ \\
\hline
\end{tabular}

and $\mathrm{Rh}_{13} \mathrm{Sc}_{57}$ from the experimental literature nearly always turn up as ground states, or very close to the convex hull, in the HT search as well. This is strong evidence that the first-principles HT approach is robust and has the necessary accuracy to extend the PGM data where experimental results are sparse or difficult to obtain. Also of interest is the appearance of some rare prototypes in 
TABLE VI. Compounds in Palladium binary alloys. The term tet-fcc denotes a tetragonal distortion of stacked fcc superstructures. All symbols are as in Table III.

\begin{tabular}{|c|c|c|c|}
\hline & \multicolumn{2}{|c|}{ Compounds } & \multirow[b]{2}{*}{$\Delta H \mathrm{meV} / \mathrm{at}$} \\
\hline & Experiments $[35,36]$ & Calculations & \\
\hline \multirow[t]{9}{*}{ Y } & $\mathrm{Pd}_{7} \mathrm{Y}\left(\mathrm{CuPt}_{7}\right)$ & $\mathrm{Pd}_{7} \mathrm{Y}\left(\mathrm{CuPt}_{7}\right)$ & -442 \\
\hline & $\mathrm{Pd}_{3} \mathrm{Y}\left(\mathrm{L1}_{2}\right)$ & $\mathrm{Pd}_{3} \mathrm{Y}\left(\mathrm{L1}_{2}\right)$ & -863 \\
\hline & $\mathrm{Pd}_{2} \mathrm{Y}$ (unkn.) & & \\
\hline & $\mathrm{Pd}_{3} \mathrm{Y}_{2}$ (unkn.) & & \\
\hline & $\mathrm{Pd}_{4} \mathrm{Y}_{3}\left(\mathrm{Pd}_{4} \mathrm{Pu}_{3}\right)$ & $\mathrm{Pd}_{4} \mathrm{Y}_{3}\left(\mathrm{Pd}_{4} \mathrm{Pu}_{3}\right)$ & -923 \\
\hline & PdY (unkn.) & PdY(B33) & -913 \\
\hline & $\mathrm{Pd}_{2} \mathrm{Y}_{3}\left(\mathrm{Ni}_{2} \mathrm{Er}_{3}\right)$ & & $\langle 8\rangle$ \\
\hline & & $\mathrm{PdY}_{2}(\mathrm{C} 37)$ & -622 \\
\hline & $\mathrm{PdY}_{3}\left(\mathrm{D0}_{11}\right)$ & $\mathrm{PdY}_{3}\left(\mathrm{D} 0_{11}\right)$ & -475 \\
\hline \multirow[t]{8}{*}{$\mathrm{Sc}$} & & $\mathrm{Pd}_{8} \mathrm{Sc}\left(\mathrm{Pt}_{8} \mathrm{Ti}\right)$ & -411 \\
\hline & & $\mathrm{Pd}_{5} \mathrm{Sc}\left(\mathrm{HfPd}_{5}^{*}\right)$ & -595 \\
\hline & $\mathrm{Pd}_{3} \operatorname{Sc}\left(\mathrm{L1}_{2}\right)$ & $\mathrm{Pd}_{3} \mathrm{Sc}\left(\mathrm{L1}_{2}\right)$ & -855 \\
\hline & $\mathrm{Pd}_{2} \mathrm{Sc}$ (unkn.) & $\mathrm{Pd}_{2} \mathrm{Sc}(\mathrm{C} 37)$ & -898 \\
\hline & & $\mathrm{Pd}_{4} \mathrm{Sc}_{3}\left(\mathrm{Pd}_{4} \mathrm{Pu}_{3}\right)$ & -910 \\
\hline & $\mathrm{PdSc}(\mathrm{B} 2)$ & $\operatorname{PdSc}(\mathrm{B} 2)$ & -906 \\
\hline & $\mathrm{PdSc}_{2}\left(\mathrm{NiTi}_{2}\right)$ & $\mathrm{PdSc}_{2}\left(\mathrm{NiTi}_{2}\right)$ & -660 \\
\hline & $\mathrm{PdSc}_{4}$ (unkn.) & & \\
\hline \multirow[t]{8}{*}{$\mathrm{Zr}$} & & $\mathrm{Pd}_{8} \mathrm{Zr}\left(\mathrm{Pt}_{8} \mathrm{Ti}\right)$ & -424 \\
\hline & & $\mathrm{Pd}_{5} \mathrm{Zr}\left(\mathrm{HfPd}_{5}^{\star}\right)$ & -591 \\
\hline & $\mathrm{Pd}_{3} \mathrm{Zr}\left(\mathrm{DO}_{24}\right)$ & $\mathrm{Pd}_{3} \mathrm{Zr}\left(\mathrm{DO}_{24}\right)$ & -816 \\
\hline & $\mathrm{Pd}_{2} \mathrm{Zr}\left(\mathrm{C} 11_{b}\right)$ & & $\langle 1\rangle$ \\
\hline & $\mathrm{Pd}_{4} \mathrm{Zr}_{3}\left(\mathrm{Pd}_{4} \mathrm{Pu}_{3}\right)$ & & $\langle 2\rangle$ \\
\hline & PdZr (unkn.) & $\operatorname{PdZr(B33)}$ & -645 \\
\hline & $\mathrm{Pd}_{3} \mathrm{Zr}_{5}\left(\mathrm{D} 8_{8}\right)$ & & $\langle 90\rangle$ \\
\hline & $\mathrm{PdZr}_{2}\left(\mathrm{NiTi}_{2} / \mathrm{CuZr}_{2}\right)$ & $\mathrm{PdZr}_{2}\left(\mathrm{C} 11_{b} / \mathrm{CuZr}_{2}\right)$ & $-487\langle 83\rangle$ \\
\hline \multirow[t]{9}{*}{ Hf } & $\begin{array}{c}\mathrm{Hf}_{2} \mathrm{Pd}\left(\mathrm{C} 11_{b} /\right. \\
\left.\quad \mathrm{CuZr}_{2}\right)\end{array}$ & $\mathrm{Hf}_{2} \mathrm{Pd}\left(\mathrm{C} 11_{b} / \mathrm{CuZr} r_{2}\right)$ & -527 \\
\hline & HfPd (unkn.) & HfPd(B33) & -685 \\
\hline & & $\mathrm{Hf}_{2} \mathrm{Pd}_{3}\left(\mathrm{Pd}_{3} \mathrm{Ti}_{2}\right)$ & -778 \\
\hline & $\mathrm{Hf}_{3} \mathrm{Pd}_{4}$ (unkn.) & & \\
\hline & & $\mathrm{Hf}_{3} \mathrm{Pd}_{5}\left(\mathrm{Pd}_{5} \mathrm{Ti}_{3}\right)$ & -800 \\
\hline & $\operatorname{HfPd}_{2}\left(\mathrm{C} 11_{b}\right)$ & & $\langle 9\rangle$ \\
\hline & $\mathrm{HfPd}_{3}\left(\mathrm{DO}_{24} / \mathrm{L1}_{2}\right)$ & $\mathrm{HfPd}_{3}\left(\mathrm{DO}_{24}\right)$ & $-879\langle 11\rangle$ \\
\hline & & $\mathrm{HfPd}_{5}\left(\mathrm{HfPd}_{5}^{\star}\right)$ & -635 \\
\hline & & $\mathrm{HfPd}_{8}\left(\mathrm{Pt}_{8} \mathrm{Ti}\right)$ & -430 \\
\hline \multirow[t]{9}{*}{$\mathrm{Ti}$} & & $\mathrm{Pd}_{5} \mathrm{Ti}\left(\mathrm{HfPd}_{5}^{\star}\right)$ & -481 \\
\hline & $\mathrm{Pd}_{3.2} \mathrm{Ti}_{0.8}\left(\mathrm{L1}_{2}\right)$ & & $\langle 7\rangle$ \\
\hline & $\mathrm{Pd}_{3} \mathrm{Ti}\left(\mathrm{DO}_{24}\right)$ & $\mathrm{Pd}_{3} \mathrm{Ti}\left(\mathrm{DO}_{24}\right)$ & -646 \\
\hline & $\mathrm{Pd}_{2} \mathrm{Ti}\left(\mathrm{Pd}_{2} \mathrm{Ti}\right)$ & $\mathrm{Pd}_{2} \mathrm{Ti}\left(\mathrm{Pd}_{2} \mathrm{Ti}\right)$ & -632 \\
\hline & $\mathrm{Pd}_{5} \mathrm{Ti}_{3}\left(\mathrm{Pd}_{5} \mathrm{Ti}_{3}\right)$ & $\mathrm{Pd}_{5} \mathrm{Ti}_{3}\left(\mathrm{Pd}_{5} \mathrm{Ti}_{3}\right)$ & -615 \\
\hline & $\mathrm{Pd}_{3} \mathrm{Ti}_{2}\left(\mathrm{Pd}_{3} \mathrm{Ti}_{2}\right)$ & $\mathrm{Pd}_{3} \mathrm{Ti}_{2}\left(\mathrm{Pd}_{3} \mathrm{Ti}_{2}\right)$ & -602 \\
\hline & PdTi(B19) & & $\langle 5\rangle$ \\
\hline & $\mathrm{PdTi}_{2}\left(\mathrm{CuZr}_{2}\right)$ & $\mathrm{PdTi}_{2}\left(\mathrm{C} 11_{b} / \mathrm{CuZr}_{2}\right)$ & -451 \\
\hline & $\mathrm{Pd}_{0.8} \mathrm{Ti}_{3.2}(\mathrm{~A} 15)$ & $\mathrm{PdTi}_{3}(\mathrm{~A} 15)$ & -342 \\
\hline \multirow[t]{2}{*}{$\mathrm{Nb}$} & & $\mathrm{Nb}_{3} \mathrm{Pd}\left(\mathrm{Nb}_{3} \mathrm{Pd}^{\dagger}\right)$ & -167 \\
\hline & & $\mathrm{Nb}_{2} \mathrm{Pd}\left(\mathrm{CuZr}_{2}\right)$ & -220 \\
\hline
\end{tabular}

TABLE VI. (Continued)

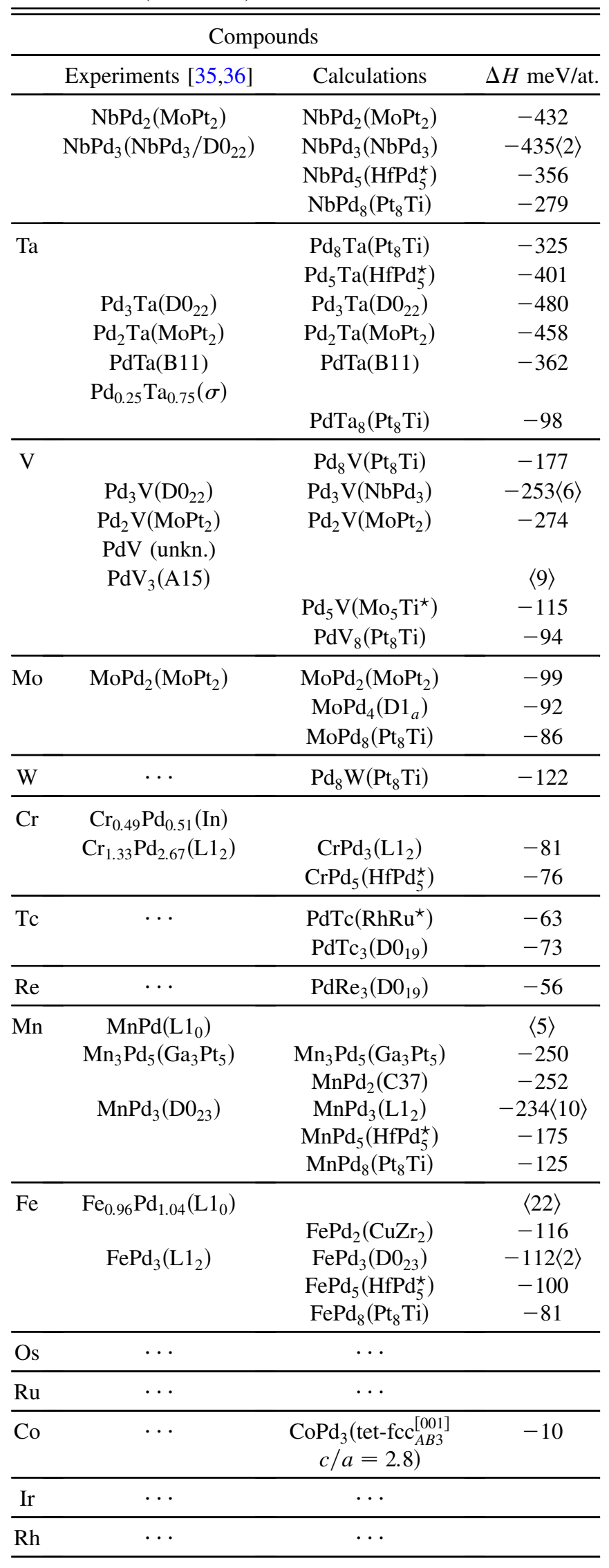


TABLE VI. (Continued)

\begin{tabular}{|c|c|c|c|}
\hline \multicolumn{4}{|c|}{ Compounds } \\
\hline & Experiments $[35,36]$ & Calculations & $\Delta H \mathrm{meV} / \mathrm{at}$. \\
\hline $\mathrm{Ni}$ & $\cdots$ & $\begin{array}{c}\mathrm{NiPd}_{3}\left(\text { tet- } \mathrm{fcc}_{A B 3}^{[001]}\right. \\
c / a=2.7)\end{array}$ & -6 \\
\hline $\mathrm{Pt}$ & $\cdots$ & $\begin{array}{c}\mathrm{Pd}_{7} \mathrm{Pt}\left(\mathrm{CuPt}_{7}\right) \\
\mathrm{Pd}_{3} \mathrm{Pt}\left(\mathrm{CdPt}_{3}^{\star}\right) \\
\operatorname{PdPt}\left(\mathrm{L1}_{1}\right) \\
\mathrm{PdPt}_{3}\left(\mathrm{L1}_{2}\right) \\
\mathrm{PdPt}_{7}\left(\mathrm{CuPt}_{7}\right) \\
\end{array}$ & $\begin{array}{l}-14 \\
-25 \\
-36 \\
-26 \\
-15 \\
\end{array}$ \\
\hline $\mathrm{Pd}$ & Reference & & \\
\hline $\mathrm{Au}$ & $\begin{array}{l}\mathrm{Au}_{3} \mathrm{Pd} \text { (unkn.) } \\
\mathrm{AuPd} \text { (unkn.) } \\
\mathrm{AuPd}_{3} \text { (unkn.) }\end{array}$ & $\begin{array}{c}\mathrm{Au}_{5} \mathrm{Pd}\left(\mathrm{HfPd}_{5}^{\star}\right) \\
\mathrm{Au}_{3} \mathrm{Pd}\left(\mathrm{DO}_{23}\right) \\
\mathrm{Au}_{2} \mathrm{Pd}(\mathrm{C} 49) \\
\mathrm{AuPd}(\mathrm{NbP}) \\
\mathrm{AuPd}_{3}\left(\mathrm{Ll}_{2}\right)\end{array}$ & $\begin{array}{l}-55 \\
-82 \\
-88 \\
-94 \\
-56\end{array}$ \\
\hline $\mathrm{Ag}$ & $\cdots$ & $\begin{array}{c}\mathrm{Ag}_{7} \mathrm{Pd}\left(\mathrm{CuPt}_{7}\right) \\
\mathrm{Ag}_{5} \mathrm{Pd}\left(\mathrm{HfPd}_{5}^{\star}\right) \\
\mathrm{Ag}_{3} \mathrm{Pd}\left(\mathrm{DO}_{23}\right) \\
\mathrm{Ag}_{2} \mathrm{Pd}(\mathrm{C} 37) \\
\mathrm{Ag}_{2} \mathrm{Pd}_{3}\left(\mathrm{Ag}_{2} \mathrm{Pd}_{3}^{\dagger}\right) \\
\left.\mathrm{AgPd} \mathrm{Li}_{1}\right) \\
\mathrm{AgPd}_{3}\left(\mathrm{CdPt}_{3}^{\star}\right)\end{array}$ & $\begin{array}{l}-33 \\
-41 \\
-58 \\
-63 \\
-63 \\
-59 \\
-31\end{array}$ \\
\hline $\mathrm{Cu}$ & $\begin{array}{c}\mathrm{Cu}_{3} \mathrm{Pd}\left(\mathrm{L1}_{2} / \mathrm{SrPb}_{3}\right) \\
\mathrm{CuPd} \text { (unkn.) } \\
\mathrm{CuPd}_{7}\left(\mathrm{CuPt}_{7}\right)\end{array}$ & $\begin{array}{c}\mathrm{Cu}_{5} \mathrm{Pd}\left(\mathrm{HfPd}_{5}^{\star}\right) \\
\mathrm{Cu}_{3} \mathrm{Pd}\left(\mathrm{DO}_{23}\right) \\
\mathrm{Cu}_{2} \mathrm{Pd}\left(\mathrm{Ga}_{2} \mathrm{Hf}\right) \\
\mathrm{CuPd}(\mathrm{B} 2) \\
\mathrm{CuPd}_{3}\left(\mathrm{Lr}_{2}\right) \\
\mathrm{CuPd}_{7}\left(\mathrm{CuPt}_{7}\right)\end{array}$ & $\begin{array}{c}\langle 18\rangle \\
-72 \\
-107\langle 2,5\rangle \\
-117 \\
-125 \\
-71 \\
-37\end{array}$ \\
\hline $\mathrm{Hg}$ & $\begin{array}{c}\mathrm{Hg}_{4} \mathrm{Pd}(\mathrm{unkn} .) \\
\mathrm{Hg}_{5} \mathrm{Pd}_{2}\left(\mathrm{Hg}_{5} \mathrm{Mn}_{2}\right) \\
\operatorname{HgPd}\left(\mathrm{L1}_{0}\right)\end{array}$ & $\begin{array}{c}\mathrm{Hg}_{4} \mathrm{Pd}\left(\mathrm{Hg}_{4} \mathrm{Pt}\right) \\
\mathrm{Hg}_{2} \mathrm{Pd}\left(\mathrm{Hg}_{2} \mathrm{Pt}\right) \\
\mathrm{HgPd}\left(\mathrm{Ll}_{0}\right) \\
\mathrm{Hg}_{3} \mathrm{Pd}_{5}\left(\mathrm{Ga}_{3} \mathrm{Pt}_{5}\right) \\
\mathrm{HgPd}_{2}(\mathrm{C} 37) \\
\mathrm{HgPd}_{3}\left(\mathrm{DO}_{22}\right) \\
\operatorname{HgPd}_{4}\left(\mathrm{D} 1_{\mathrm{a}}\right)\end{array}$ & $\begin{array}{l}-101 \\
\langle 65\rangle \\
-150 \\
-174 \\
-166 \\
-160 \\
-139 \\
-112\end{array}$ \\
\hline $\mathrm{Cd}$ & $\begin{array}{c}\mathrm{Cd}_{11} \mathrm{Pd}_{2}\left(\mathrm{Ir}_{2} \mathrm{Zn}_{11}\right) \\
\mathrm{Cd}_{4} \mathrm{Pd} \text { (unkn.) } \\
\mathrm{Cd}_{3} \mathrm{Pd} \text { (unkn.) }\end{array}$ & $\begin{array}{c}\mathrm{Cd}_{2} \mathrm{Pd}\left(\mathrm{Hg}_{2} \mathrm{Pt}\right) \\
\mathrm{CdPd}\left(\mathrm{L1}_{0}\right) \\
\mathrm{CdPd}_{2}(\mathrm{C} 37) \\
\mathrm{CdPd}_{3}\left(\mathrm{D} 0_{22}\right) \\
\mathrm{CdPd}_{4}\left(\mathrm{D}_{a}\right) \\
\mathrm{CdPd}_{5}\left(\mathrm{HfPd}_{5}^{\star}\right) \\
\mathrm{CdPd}_{7}\left(\mathrm{CuPt}_{7}\right) \\
\end{array}$ & $\begin{array}{c}-307 \\
-418\langle 164\rangle \\
-334 \\
-272 \\
-225 \\
-188 \\
-142 \\
\end{array}$ \\
\hline $\mathrm{Zn}$ & $\mathrm{Pd}_{2} \mathrm{Zn}(\mathrm{C} 37)$ & $\begin{array}{c}\mathrm{Pd}_{8} \mathrm{Zn}\left(\mathrm{Pt}_{8} \mathrm{Ti}\right) \\
\mathrm{Pd}_{2} \mathrm{Zn}(\mathrm{C} 37)\end{array}$ & $\begin{array}{l}-165 \\
-462\end{array}$ \\
\hline
\end{tabular}

TABLE VI. (Continued)

\begin{tabular}{ccc}
\hline \hline \multicolumn{3}{c}{ Compounds } \\
\hline Experiments [35,36] & Calculations & $\Delta H$ meV/at. \\
\hline $\mathrm{PdZn}(\mathrm{CuTi})$ & $\mathrm{PdZn}\left(\mathrm{L} 1_{0}\right)$ & $-570<187\rangle$ \\
$\mathrm{Pd}_{3} \mathrm{Zn}_{5}$ (unkn.) & & -359 \\
& $\mathrm{PdZn}_{3}\left(\mathrm{DO}_{22}\right)$ & -243 \\
$\mathrm{Pd}_{2} \mathrm{Zn}_{11}\left(\mathrm{Ir}_{2} \mathrm{Zn}_{11}\right)$ & $\mathrm{Pd}_{2} \mathrm{Zn}_{11}\left(\mathrm{Ir}_{2} \mathrm{Zn}_{11}\right)$ & \\
\hline
\end{tabular}

systems similar to those in which they were identified experimentally. For example, the prototype $\mathrm{Pd}_{3} \mathrm{Ti}_{2}$, reported only in the Pd-Ti system [36], also emerges as a calculated ground state in the closely related system Hf-Pd. In Hf-Pt, it appears as marginally stable, at $3 \mathrm{meV} /$ atom above the convex hull, in agreement with a very recent experimental study that identified the previously incorrectly characterized structure of a $\mathrm{Hf}_{2} \mathrm{Pt}_{3}$ phase [46].

In the systems we examined, there are nearly 50 phases reported in the experimental phase diagrams for which the crystal structure of the phase is not known. In half of these cases, the HT calculations identify stable structures for these unknown phases. For the other half of these unknown structures, our calculations find no stable compounds at the reported concentration, but find stable compounds at other concentrations. The reported phases (sans structural information) may, therefore, be due to phases that decompose at low temperatures or may merely represent samples that were kinetically inhibited and unable to settle into their stable phases during the time frame of the experiments.

The prototype database included in this study comprises both experimentally reported structures and hypothetical structures constructed combinatorially from derivative supercells of fcc, bcc, and hcp lattices [39,47]. Occasionally, these derivative superstructures are predicted to be ground states by the first-principles calculations. In this work, we find compounds with five of these new structures, for which no prototype is known and no Strukturbericht designation has been given. These new prototypes are marked by a $†$ in Tables III, IV, V, and VI, and their crystallographic parameters are given in Table VII. We also find a few other compounds with unobserved prototypes (marked by a $\star$ in Tables I, II, III, IV, V, and VI) previously uncovered in related HT studies $[2,13,25,27,29,31]$.

\section{STRUCTURE MAPS}

Empirical structure maps present available experimental data in ways that highlight similarities in materials behavior in alloy systems. Their arrangement principles usually depend on simple parameters, e.g., atomic number, atomic radius, electronegativity, ionization energy, melting temperature, or enthalpy. Several well-known classification methods include Hume-Rothery rules 
TABLE VII. Geometry of new prototypes marked by $†$ in Tables III, IV, V, and VI.

\begin{tabular}{|c|c|c|c|c|c|}
\hline Formula & IrZn & $\mathrm{Nb}_{3} \mathrm{Pd}$ & $\mathrm{Fe}_{2} \mathrm{Rh}$ & $\mathrm{Ag}_{3} \mathrm{Pt}_{2}$ & $\mathrm{Ag}_{2} \mathrm{Pd}_{3}$ \\
\hline Lattice & Monoclinic & Orthorhombic & Orthorhombic & Rhombohedral & Monoclinic \\
\hline Space Group & $C 2 / m$ No. 12 & Cmmm No. 65 & Cmmm No. 65 & $R \overline{3} m$ No. 166 & $C 2 / m$ No. 12 \\
\hline Pearson symbol & $\mathrm{mS} 8$ & oS8 & oS12 & $\mathrm{hR5}$ & $\mathrm{mS} 10$ \\
\hline Bravais lattice type & MCLC & ORCC & ORCC & RHL & MCLC \\
\hline Lattice variation [48] & $\mathrm{MCLC}_{1}$ & ORCC & ORCC & $\mathrm{RHL}_{1}$ & $\mathrm{MCLC}_{3}$ \\
\hline Conv. Cell: $a, b, c(\AA)$ & $1.94,3.83,1.12$ & $1.26,1.78,3.56$ & $1.78,5.35,1.26$ & $1.12,1.12,13.75$ & $3.55,1.59,1.94$ \\
\hline$\alpha, \beta, \gamma(\mathrm{deg})$ & $72.98,90,90$ & $90,90,90$ & $90,90,90$ & $90,90,120$ & $65.9,90,90$ \\
\hline Wyckoff & $\operatorname{Ir} \frac{1}{6}, \frac{1}{2},-0.292$ & $\mathrm{Nb} 10,0, \frac{1}{4}(4 \mathrm{k})$ & $\mathrm{Fe} 1 \frac{1}{6}, 0,0(4 \mathrm{~g})$ & $\mathrm{Ag} 10,0, \frac{1}{5}(2 \mathrm{c})$ & $\operatorname{Ag} \frac{3}{10}, \frac{1}{2}, \frac{1}{10}(4 i)$ \\
\hline \multirow[t]{2}{*}{ Positions [49] } & $\mathrm{Zn} \frac{1}{6}, \frac{1}{2},-0.208(4 \mathrm{i})$ & $\mathrm{Nb} 2 \frac{1}{2}, 0, \frac{1}{2}(2 \mathrm{c})$ & $\mathrm{Fe} 20,0, \frac{1}{2}(2 \mathrm{~d})$ & $\operatorname{Ag} 20,0,0$ (1a) & $\operatorname{Pd} 10,0, \frac{1}{2}(2 \mathrm{c})$ \\
\hline & & $\operatorname{Pd} \frac{1}{2}, 0,0(2 b)$ & $\begin{array}{c}\mathrm{Fe} 3 \frac{1}{2}, 0,0(2 \mathrm{~b}) \\
\mathrm{Rh} \frac{1}{3}, 0, \frac{1}{2}(4 \mathrm{~h})\end{array}$ & Pt $0,0, \frac{2}{5}(2 \mathrm{c})$ & $\operatorname{Pd} 2 \frac{1}{10}, \frac{1}{2}, \frac{7}{10}(4 \mathrm{i})$ \\
\hline AFLOW label [33] & 123 & 72 & b83 & $\mathrm{f} 38$ & f55 \\
\hline
\end{tabular}

[50], Miedema formation enthalpy [51], Zunger pseudopotential radii maps [52], and Pettifor maps [44,45]. These empirical rules and structure maps have helped direct a few successful searches for previously unobserved compounds [53]. However, they offer a limited response to the challenge of identifying new compounds because they rely on the existence of consistent and reliable experimental input for systems spanning most of the relevant parameter space. In many cases, reliable information is missing in a large portion of this space; e.g., less than $50 \%$ of the binary systems have been satisfactorily characterized [54]. This leaves considerable gaps in the empirical structure maps and reduces their predictive usefulness. The advance of HT computational methods makes it possible to fill these gaps in the experimental data with complementary $a b$ initio data by efficiently covering extensive lists of candidate structure types [28]. This development was envisioned by Pettifor a decade ago [53], and here we present its realization for PGM alloys.

Figure 2 shows a Pettifor structure map, enhanced by our HT computational results, for structures of 1:1 stoichiometry. The elements along the map axes are ordered according to Pettifor's chemical scale ( $\chi$ parameter) [45]. Circles indicate agreement between computation and experiment, regarding the existence of 1:1 compounds, or lack thereof. If the circle contains a label (Strukturbericht or prototype), this denotes the structure that is stable in the given system at this stoichiometry. Rectangles denote disagreement between experiments and computation about the 1:1 compounds, in systems reported as compound forming (blue rectangles) or as non-compound forming (red and gray rectangles). In the lower left part of the map, there is a region of noncompound-forming systems, whereas the upper part of the map is mostly composed of compound-forming systems. In the upper part of the map, experiment and computation agree, preserving a large cluster of B2 structures, or differ slightly on the structure reported to have the lowest formation enthalpy at 1:1 (blue rectangles). For example, the 1:1 phases of Hf-Pd and $\mathrm{Pd}-\mathrm{Zr}$ are unknown according to the phase-diagram literature, but we find the stable phases with B33 structure, right next to Hf-Pt in the diagram, which is reported as a B33 structure. Similarly, stable $\mathrm{L}_{0}$ structures are identified in the $\mathrm{Ir}-\mathrm{Ti}$ and $\mathrm{Rh}-\mathrm{Ti}$ systems, adjacent to a reported cluster of this structure. Two additional $\mathrm{Ll}_{0}$ structures are identified in the Cd-Pd and Pd-Zn systems, instead of the reported CuTi structures, extending a small known cluster of this structure at the bottom right corner of the map. These are examples of the capability of HT $a b$ initio results to complement the empirical Pettifor maps and extend their regions of predictive input, in a way consistent with the experimental data.

In the middle of the map, in a rough transition zone between compound-forming and non-compound-forming regions, computation finds quite a few cases where stable compounds are predicted in systems where none have been reported experimentally (pink rectangles). Most prominent here is a large cluster of B19 compounds. Nine systems marked by light gray rectangles are reported in experiments as having no compounds, but our calculations find stable compounds at stoichiometries other than $1: 1$.

At the stoichiometries of 1:2 and 2:1, Fig. 3 shows significant additions of the calculations to the experimental data on compound formation. Again, the systems where computation finds stable compounds in experimentally non-compound-forming systems are found at the border between the compound-forming region (dark gray circles and white labeled circles) and the non-compound-forming region (light gray circles), or they fill isolated gaps within the compound-forming regions. The calculations augment 


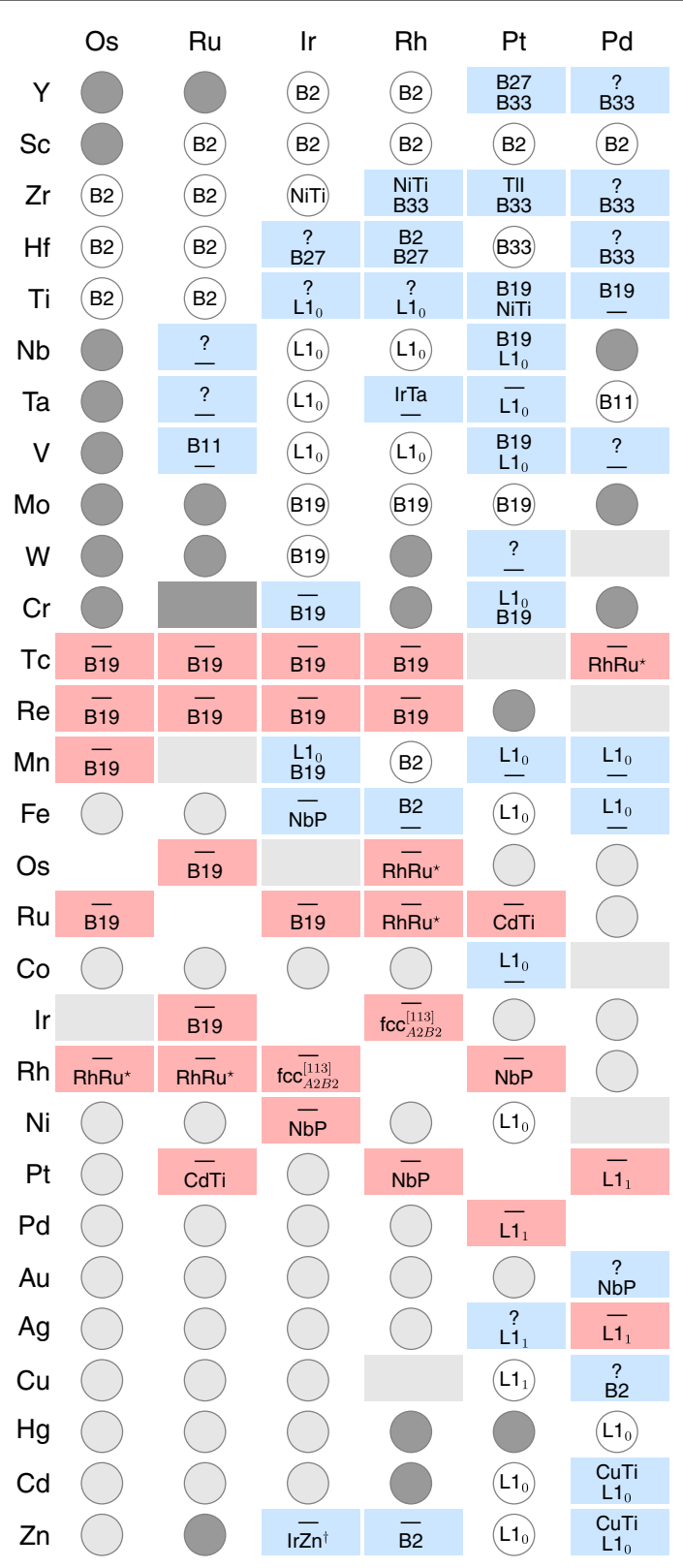

FIG. 2. A Pettifor-type structure map for 1:1 stoichiometry compounds in PGM binary systems. Circles indicate agreement between experiment and computation: White circles with Strukturbericht or prototype labels denote 1:1 compounds, dark circles indicate a compound-forming system with no compounds at $1: 1$, and light circles denote non-compound-forming systems. Blue rectangles denote compound-forming systems where the reported and computed stable structures differ at 1:1 stoichiometry. The top label in the rectangle is the reported structure; the bottom label is the structure we find to be stable in this work. A dash "_." indicates the absence of a stable structure. Unidentified suspected structures are denoted by a question mark. Pink rectangles indicate systems reported as non-compound forming, with a dash at the top of the rectangle, but we find a stable 1:1 phase, identified at the bottom of the rectangle. Light gray rectangles indicate systems reported as non-compound forming where a structure is predicted at a stoichiometry different from 1:1. A dark gray rectangle indicates a system reported with a disordered compound where no stable structures are found in the calculation. islands of structurally similar regions, yielding a more consistent structure map. For example, calculation finds the $\mathrm{CuZr}_{2}$ structure for $\mathrm{Nb}-\mathrm{Pd}$, extending the island of this structure already present in the experimental results (left panel, upper right). The calculations significantly extend the $\mathrm{Hg}_{2} \mathrm{Pt}$ island in the lower right of the $\mathrm{B}_{2} \mathrm{~A}$ panel, from a single experimental entry to six systems (in $\mathrm{Hg}-\mathrm{Pt}$ itself, the calculation finds this structure slightly unstable at $T=0 K, 25 \mathrm{meV} /$ atom above the stability tieline). A cluster of $\sigma$ phases in the left panel shows that this reported disordered phase has underlying ordered realizations at low temperatures. Three completely new islands, for the $\mathrm{C} 37, \mathrm{Ga}_{2} \mathrm{Hf}$, and $\mathrm{IrTc}_{2}$ structures, appear near the upper center of the $\mathrm{A}_{2} \mathrm{~B}$ panel. Another new cluster, of the $\mathrm{Pd}_{2} \mathrm{Ti}$ structure, appears at the lower center of both panels. In general, the clusters of blue rectangles show that the calculations augment the experimental results in a consistent manner.

The structure map for 1:3 phases is shown in Fig. 4. Similarly to the $1: 1$ and 1:2 maps, the calculation extends structural islands of the experimental data, most new phases in non-compound-forming systems occur in systems at the boundary between compound-forming and non-compound-forming regions, and there is significant agreement between the experimentally reported phases (or lack thereof) and calculated phases. In the upper part of the right panel, the $\mathrm{L}_{2}$ and $\mathrm{DO}_{24}$ clusters are preserved with slight modifications at their boundaries (at $\mathrm{Pt}-\mathrm{Ti}$, the $\mathrm{PuAl}_{3}$ structure is only $3 \mathrm{meV} /$ atom lower than the experimental structure $\mathrm{DO}_{24}$, a difference too small to be significant). The $\mathrm{D}_{19}$ cluster is significantly expanded. In the left panel, the calculations introduce a new $\mathrm{D}_{19}$ island near the center of the diagram. New small regions of the $\mathrm{DO}_{22}$ structures emerge at the right bottom of both panels. Adjacent $\mathrm{DO}_{23}$ and $\mathrm{CdPt}_{3}^{\star}$ islands appear in the left and right panels, respectively. The experimental $\mathrm{DO}_{e}$ structure for $\mathrm{RhZr}_{3}$ may actually be $\mathrm{SV}_{3}$ since in the calculation the $\mathrm{D} 0_{e}$ structure relaxed into the $\mathrm{SV}_{3}$ structure, creating a small $\mathrm{SV}_{3}$ island at the top of the left panel.

The structure maps of Figs. 2-4 give a bird's eye view of the exhaustive HT search for new structures. Consistent with the empirical maps, they show significant separation of different structures into regions where the constituent elements have a similar Pettifor $\chi$ number. The HT data significantly enhance the empirical maps, extend the regions of some structures, fill in apparent gaps, and indicate previously unsuspected structure clusters. Moreover, the HT data contain more detail than is apparent in the structure maps. Even when calculation and experiment agree that a system is compound forming [green (dark gray) circles in Fig. 1], the calculations often find additional stable compounds, beyond those known in experiment. When the reported structures are found to be unstable in the calculation, they are 


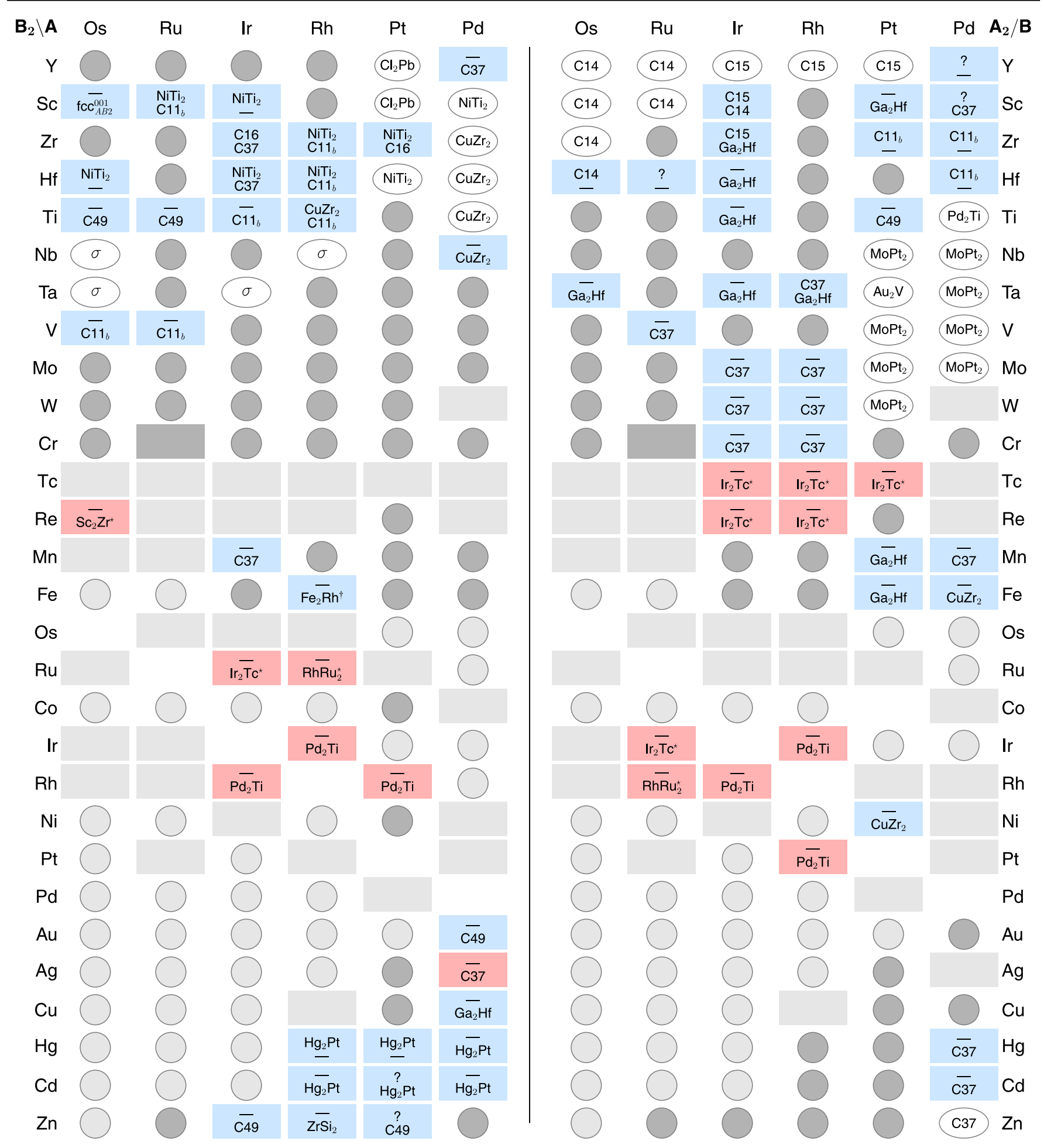

FIG. 3. A Pettifor-type structure map for 1:2 stoichiometry compounds in PGM binary systems. The symbols are as in Fig. 2, with the map stoichiometry changed, respectively, from 1:1 to 1:2 or 2:1.

usually just slightly less stable than the calculated ground state, or just slightly above the convex hull in a two-phase region. Such cases and numerous additional predictions of marginally stable structures harbor further opportunities for materials engineering and applications.

\section{CONCLUSIONS}

In this study, the low-temperature phase diagrams of all binary PGM-transition metal systems are constructed by HT $a b$ initio calculations. The picture of PGM alloys emerging from this study is much more complete than 


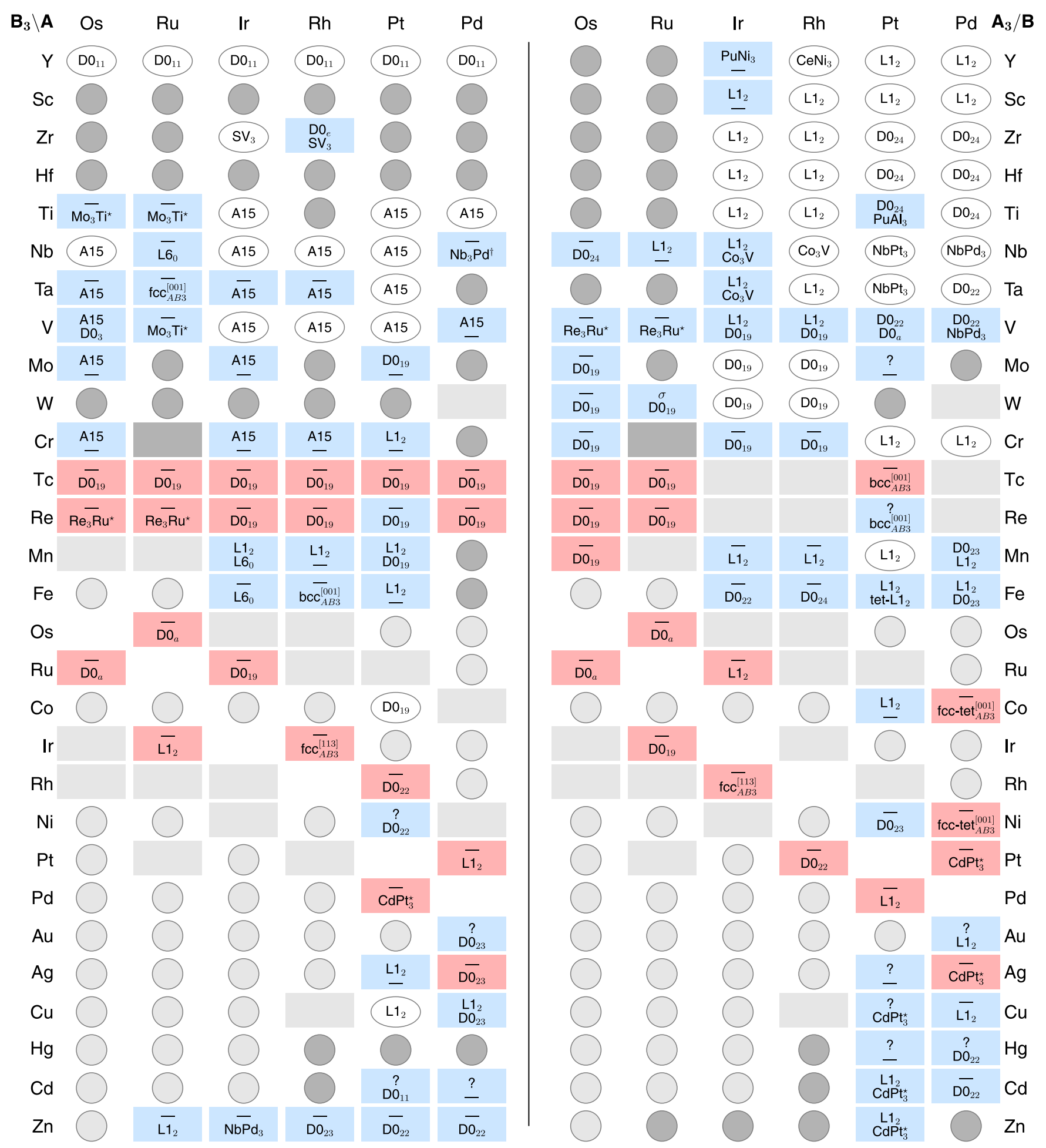

FIG. 4. A Pettifor-type structure map for 1:3 stoichiometry compounds in PGM binary systems. The symbols are as in Fig. 2.

that depicted by current experimental data, with dozens of stable structures that have not been previously reported. We predict ordering in 28 systems reported to be phase separating and in five systems where only disor- dered phases are reported. In addition, in the known ordering systems, we find many cases in which more phases are predicted to be stable than reported in the experimental phase diagrams. These ab initio results 
complement the ordering tendencies implied by the empirical Pettifor maps. Augmenting the experimental data compiled in the phase-diagram databases $[35,36]$ with high-throughput first-principles data [33,34], we construct Pettifor-type structure maps that point to new opportunities for alloys research. These maps demonstrate that the integration of the empirical and computational data produces enhanced maps that should provide a more comprehensive foundation for rational materials design. The theoretical predictions presented here will hopefully serve as a motivation for their experimental validation and be a guide for future studies of these important systems.

The maps in Figs. 2-4 include a large number of light blue rectangles, pointing to experiment-theory mismatches on structures at simple compositions in binary systems known to be compound forming. This may raise reservations that the level of theory employed, DFTPBE, may not be as good as commonly accepted for transition metal alloys. A more careful look, however, shows that many of these mismatches, e.g., HfIr, PdZr, $\mathrm{Cd}_{2} \mathrm{Pt}, \mathrm{CuPt}_{3}$, and $\mathrm{Au}_{3} \mathrm{Pd}$, involve cases where a compound of unknown structure has been reported by experiments. The calculation thus reveals the stable structure and closes the gap in the experimental data. In most other cases, e.g., $\mathrm{RhZr}, \mathrm{PtV}, \mathrm{Ir}_{3} \mathrm{~V}, \mathrm{Rh}_{2} \mathrm{Ta}$, and $\mathrm{Cu}_{3} \mathrm{Pt}$, the energy difference between the reported structure and the calculated structure or two-phase tieline is rather small and is congruent with the adjacent structure clusters in the maps. Similar improved consistency with reported structure clusters also appears in cases where the discrepancies are considerable, e.g., CdPd and PdZn. In addition, as discussed in Sec. III, the calculations reproduce many complex large unit-cell structures that are reported in the experimental literature. Moreover, it is important to remember that experiments are performed at room temperature or higher, while our calculations are carried out at zero temperature. Many phase discrepancies may therefore be due to vibrational promotion [55] or the tendency of structures to gain symmetries by losing their internal Peierls instabilities or Jahn-Teller distortions. Therefore, the disagreements emerging in our calculations may not be a sign of deficiencies in the theoretical treatment but a demonstration of its usefulness is bridging gaps in the experimental data and extending it towards unknown phase transitions at lower temperatures. The ultimate test of this issue rests with experimental validation of at least some of our predictions, which would hopefully be motivated by this work.

To help accelerate this process of experimental validation, discovery, and development of materials [56], we will offer a public domain "Application Programming Interface" (REST-API [57]) that will allow the scientific community to download information from the AFLOWLIB repository [37]. It would ultimately enable researchers to generate alloy information remotely on their own computers. Extension of the database to nanoalloys and nanosintered systems is planned within the size-pressure approximation (i.e., Fig. 2 of Ref. [58]), to study trends of solubility and size-dependent disorder-order transitions and segregation in nanocatalysts [21,58-60] and nanocrystals $[61,62]$.

A few of our predictions correspond to phases where the driving force for ordering is small (i.e., the formation enthalpy is small, and it may be difficult to reach thermal equilibrium); however, it should be noted that some experimentally reported phases have similarly small formation enthalpies. Some of these predicted phases could be more easily realized as nanostructured phases, where the thermodynamics for their formation may be more favorable. Our results should serve as the foundation for finite-temperature simulations to identify phases that are kinetically accessible. Rapid thermodynamical modeling and descriptor-based screening of systems predicted to harbor new phases should be used to pinpoint those phases with the greatest potential for applications [28]. Such simulations would be an invaluable extension to this work; however, the necessary tools to accomplish them on a similarly large scale are not yet mature.

\section{ACKNOWLEDGMENTS}

S. C. acknowledges support from DOD-ONR (Grants No. N00014-13-1-0635, No. N00014-11-1 0136, and No. N00014-09-1-0921). G. L.W.H. is grateful for support from the National Science Foundation, Grant No. DMR-0908753. O.L. thanks the Center for Materials Genomics of Duke University for its hospitality. We thank Fulton Supercomputing Laboratory and the Cray Corporation for computational support. The authors thank Dr. M. Buongiorno Nardelli, Dr. M. Asta, Dr. C. Toher, Dr. K. Rasch, and Dr. C.E. Calderon for useful comments.

Note added in proof.-While this paper was being prepared for publication, a new experimental study identified the new compound $\mathrm{Hf}_{3} \mathrm{Pt}_{4}$, of prototype $\mathrm{Pd}_{4} \mathrm{Pu}_{3}$ [63]. Our calculations show that this structure is metastable in the HfPt system, at $19 \mathrm{meV} / \mathrm{atom}$ above the convex hull, as well as in the HfPd and PdZr systems. The same structure is predicted as a ground state in the RhZr, PtSc, PdSc, PtY, and PdY systems.

\section{APPENDIX}

This appendix includes Figs. 5-19, which present the low-temperature phase diagrams (convex hulls) of all the compound-forming PGM-transition metal binary systems. 

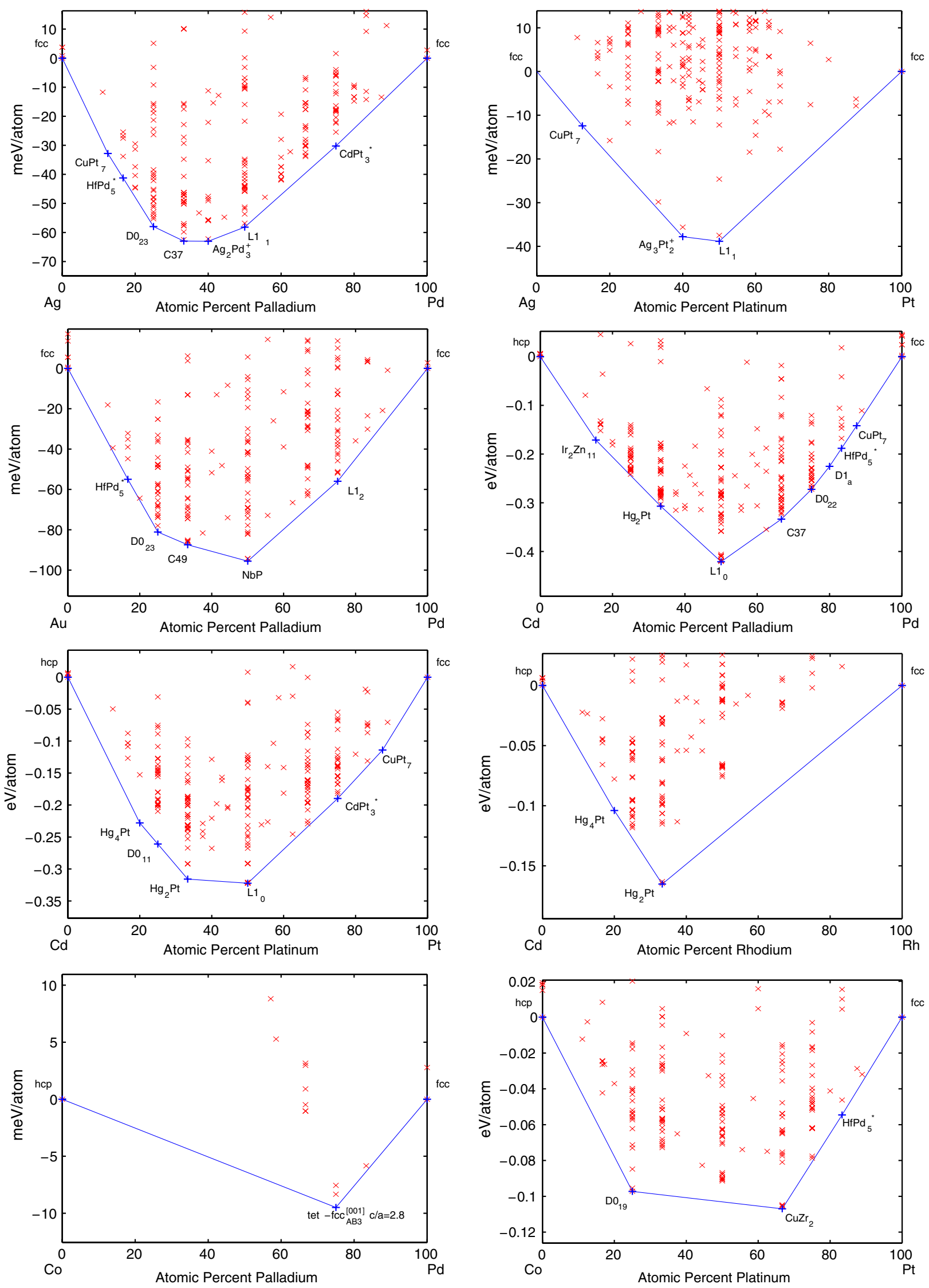

FIG. 5. Convex hulls for the systems Ag-Pd, Ag-Pt, Au-Pd, Cd-Pd, Cd-Pt, Cd-Rh, Co-Pd, and Co-Pt. 

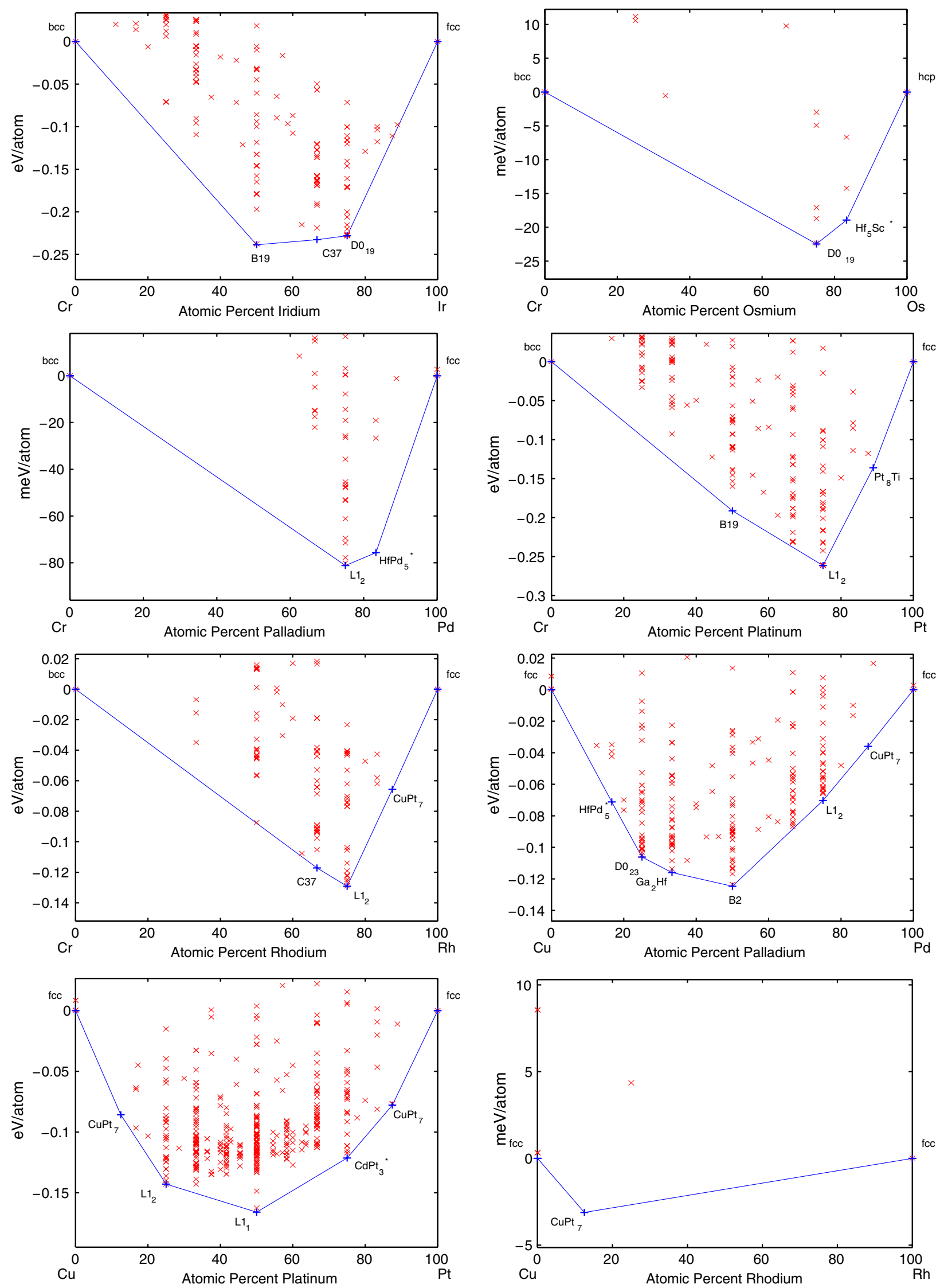

FIG. 6. Convex hulls for the systems Cr-Ir, Cr-Os, Cr-Pd, Cr-Pt, Cr-Rh, Cu-Pd, Cu-Pt, and Cu-Rh. 

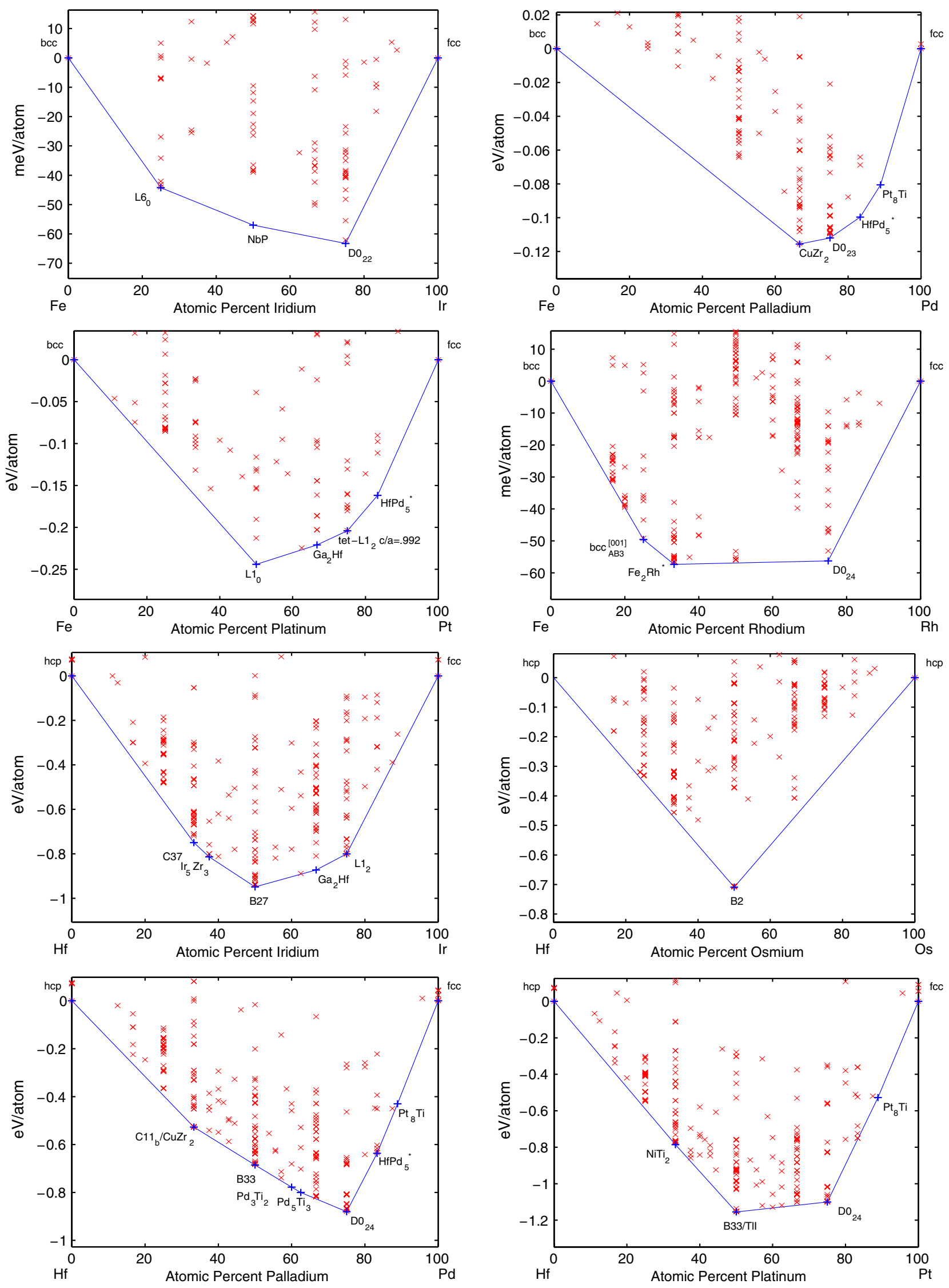

FIG. 7. Convex hulls for the systems Fe-Ir, Fe-Pd, Fe-Pt, Fe-Rh, Hf-Ir, Hf-Os, Hf-Pd, and Hf-Pt. 

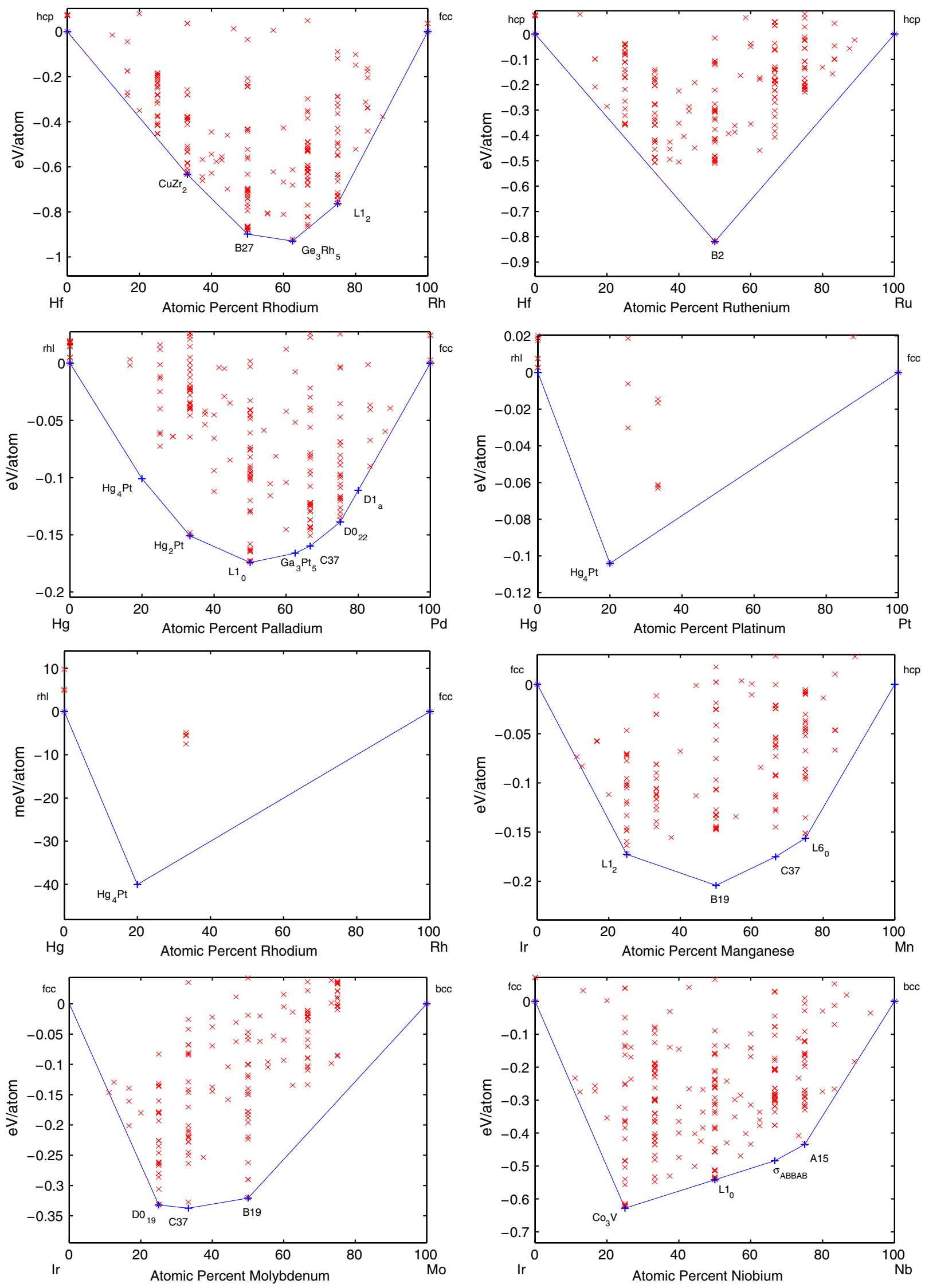

FIG. 8. Convex hulls for the systems Hf-Rh, Hf-Ru, Hg-Pd, Hg-Pt, Hg-Rh, Ir-Mn, Ir-Mo, and Ir-Nb. 

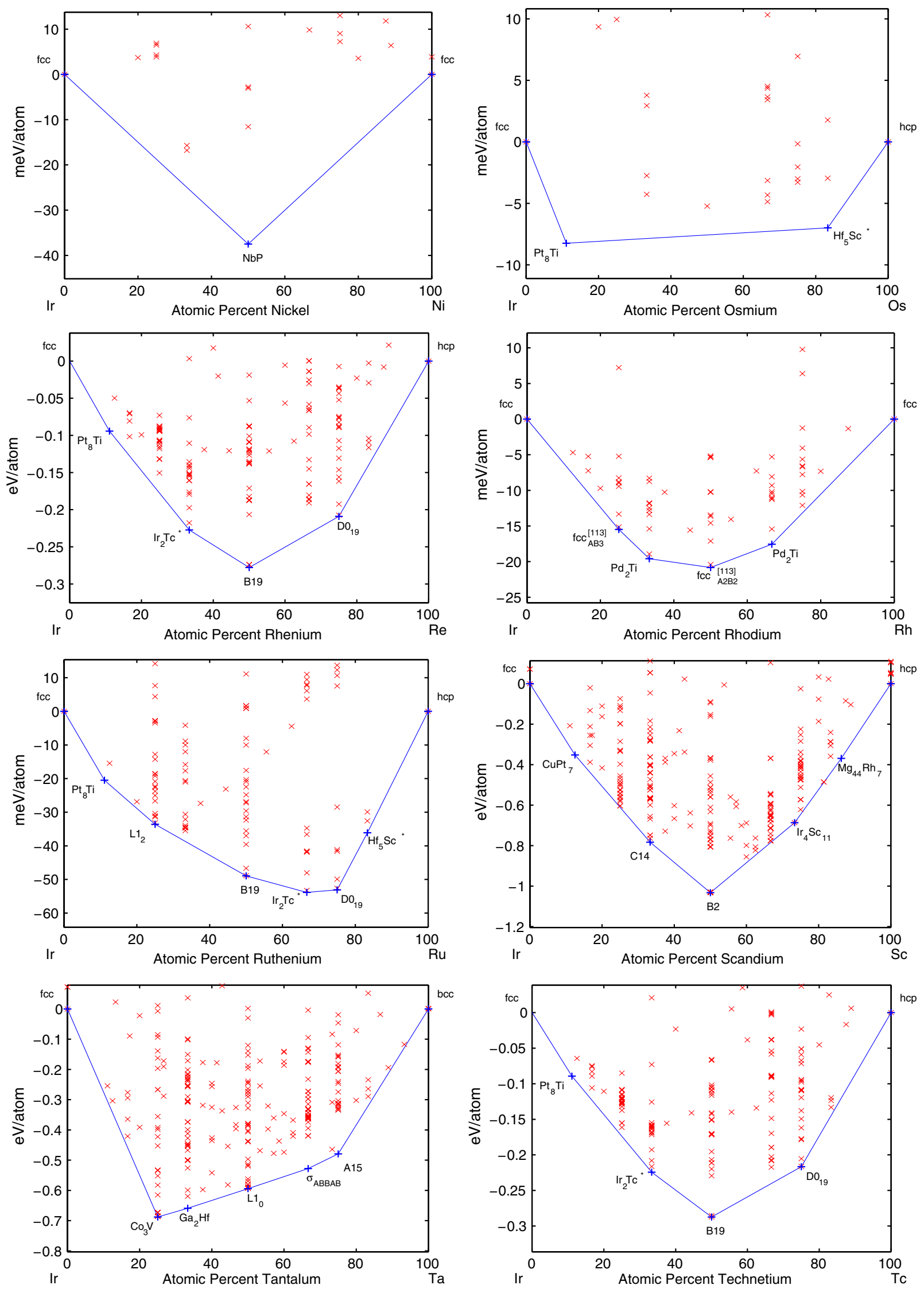

FIG. 9. Convex hulls for the systems Ir-Ni, Ir-Os, Ir-Re, Ir-Rh, Ir-Ru, Ir-Sc, Ir-Ta, and Ir-Tc. 

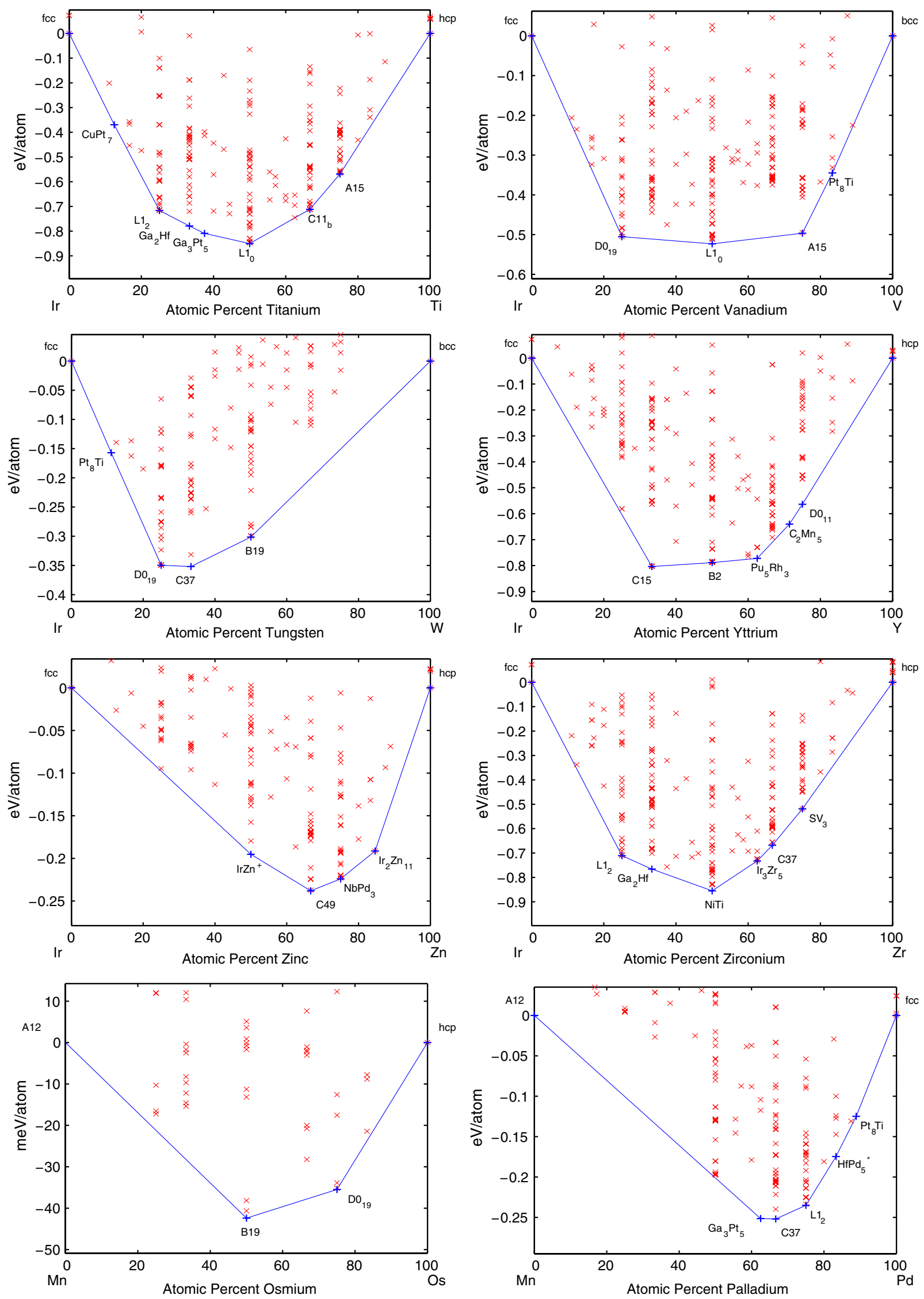

FIG. 10. Convex hulls for the systems Ir-Ti, Ir-V, Ir-W, Ir-Y, Ir-Zn, Ir-Zr, Mn-Os, and Mn-Pd. 

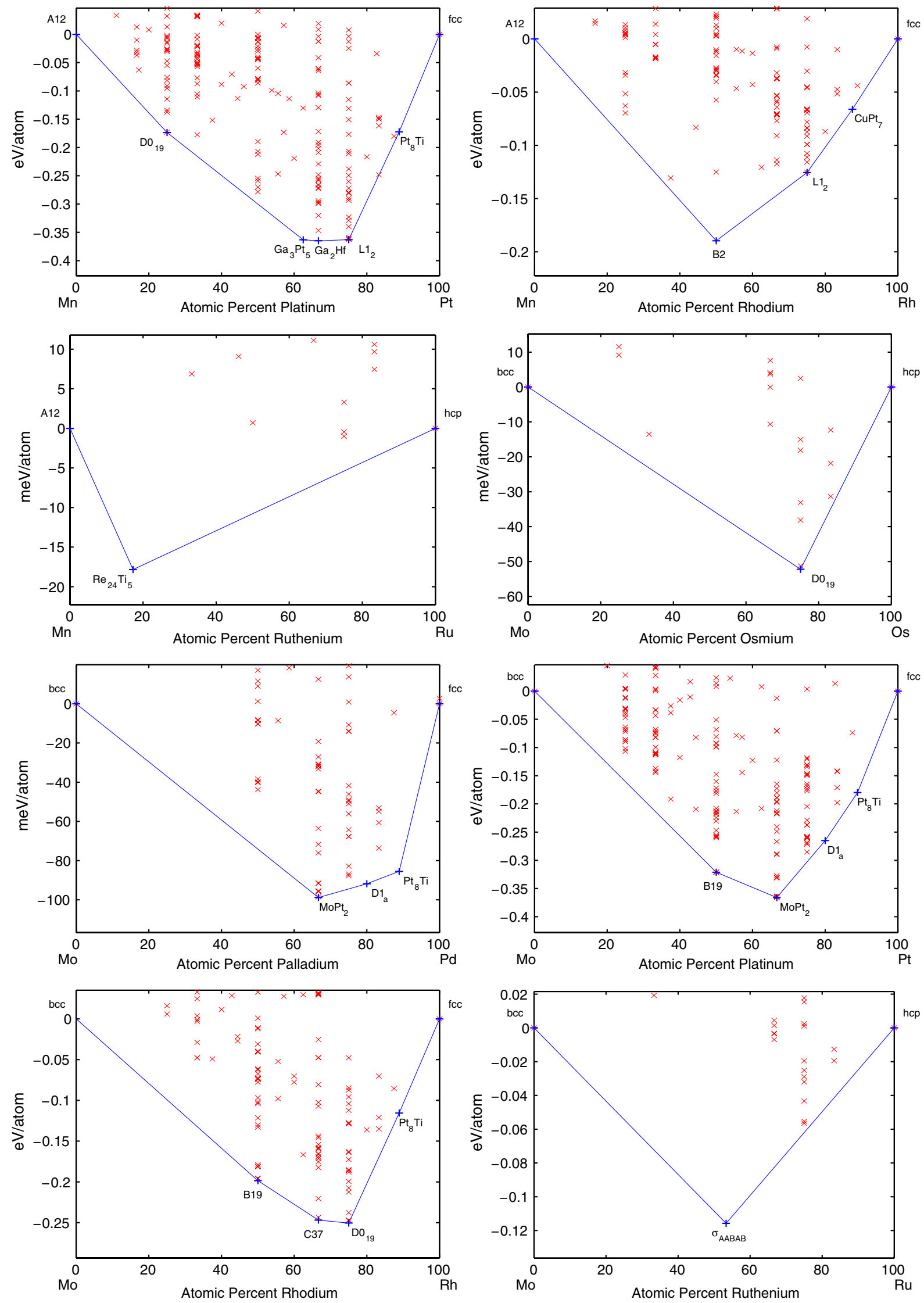

FIG. 11. Convex hulls for the systems Mn-Pt, Mn-Rh, Mn-Ru, Mo-Os, Mo-Pd, Mo-Pt, Mo-Rh, and Mo-Ru. 

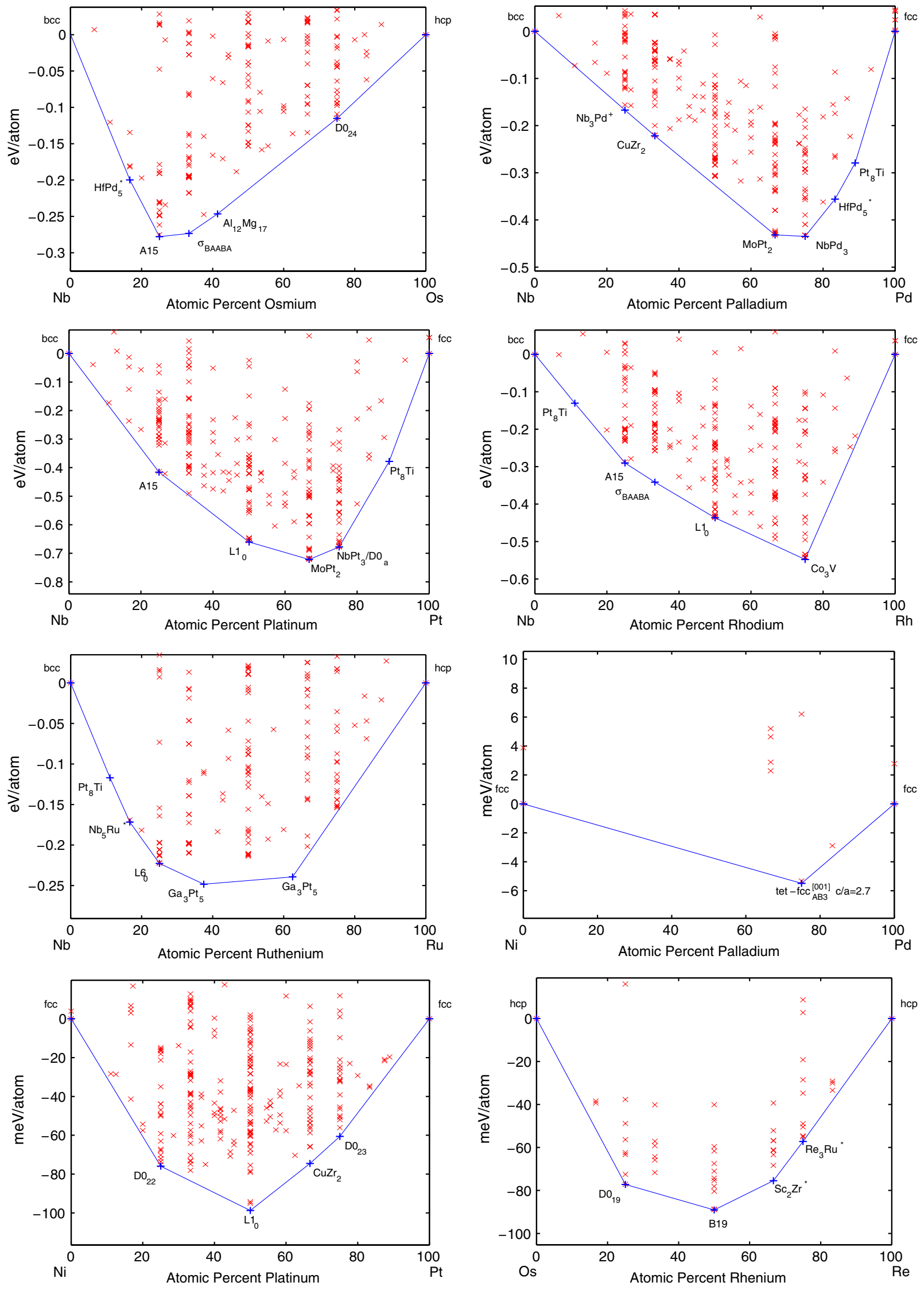

FIG. 12. Convex hulls for the systems Nb-Os, Nb-Pd, Nb-Pt, Nb-Rh, Nb-Ru, Ni-Pd, Ni-Pt, and Os-Re. 

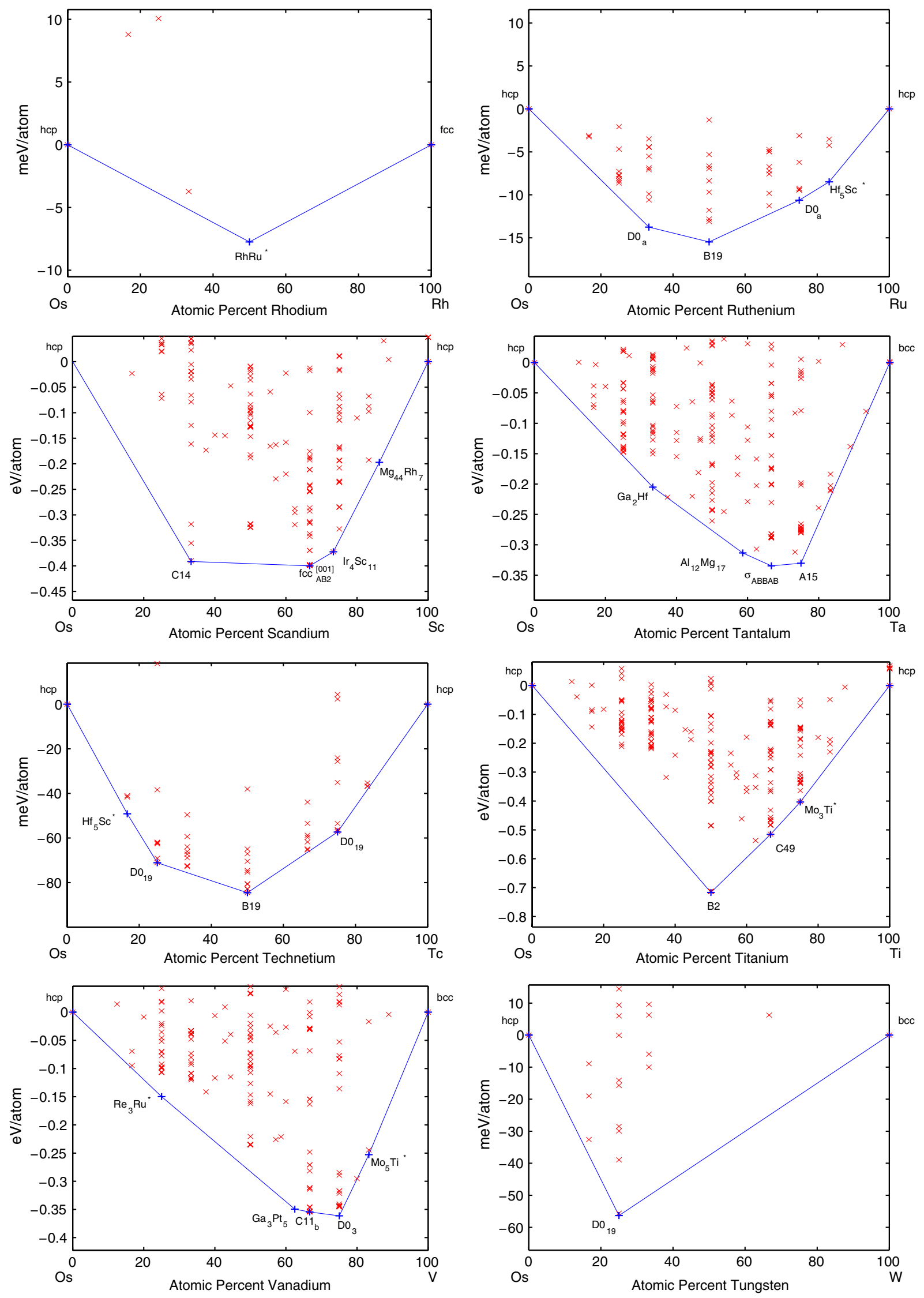

FIG. 13. Convex hulls for the systems Os-Rh, Os-Ru, Os-Sc, Os-Ta, Os-Tc, Os-Ti, Os-V, and Os-W. 

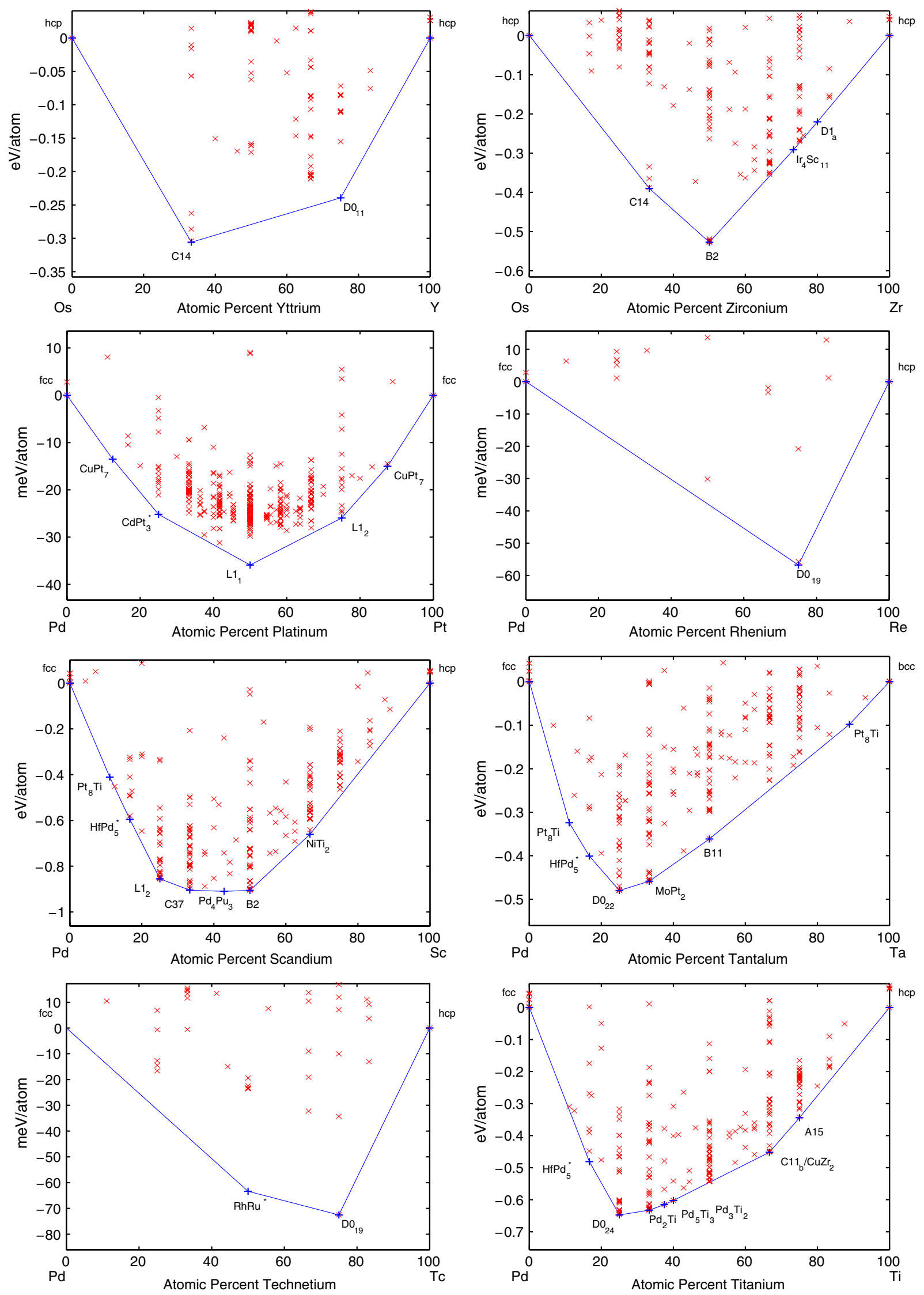

FIG. 14. Convex hulls for the systems Os-Y, Os-Zr, Pd-Pt, Pd-Re, Pd-Sc, Pd-Ta, Pd-Tc, and Pd-Ti. 

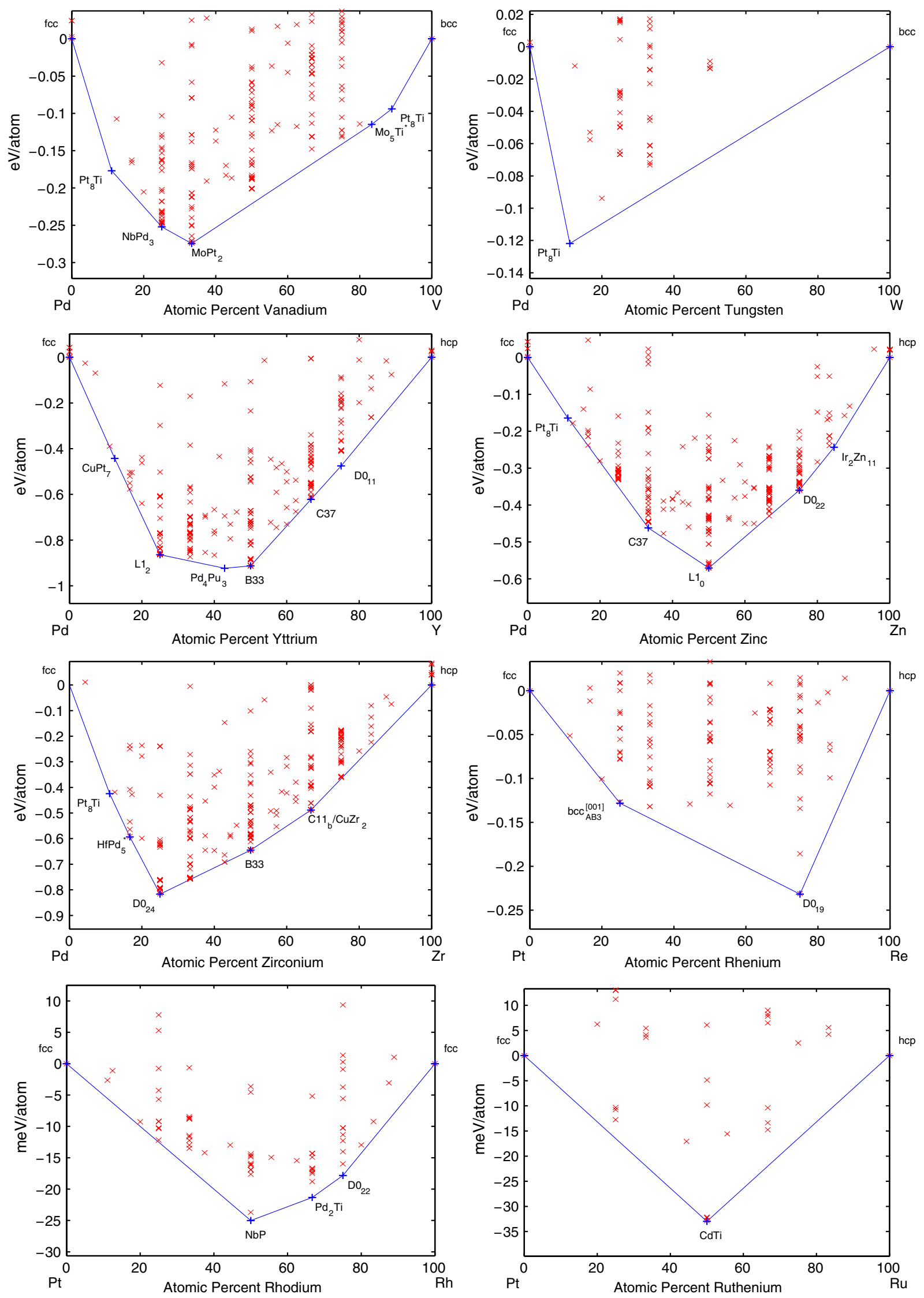

FIG. 15. Convex hulls for the systems PdV, Pd-W, Pd-Y, Pd-Zn, Pd-Zr, Pt-Re, Pt-Rh, and Pt-Ru. 

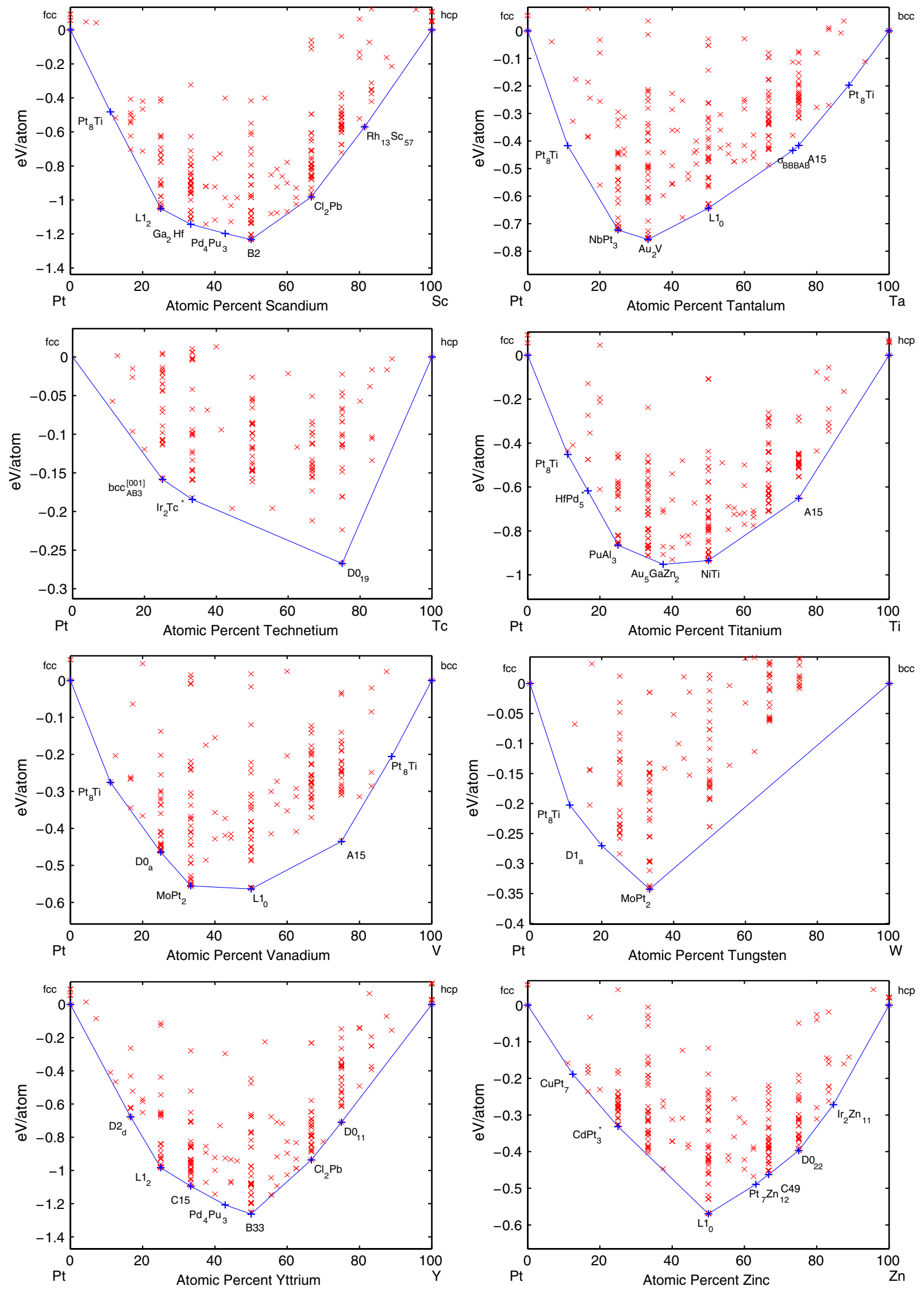

FIG. 16. Convex hulls for the systems Pt-Sc, Pt-Ta, Pt-Tc, Pt-Ti, Pt-V, Pt-W, Pt-Y, and Pt-Zn. 

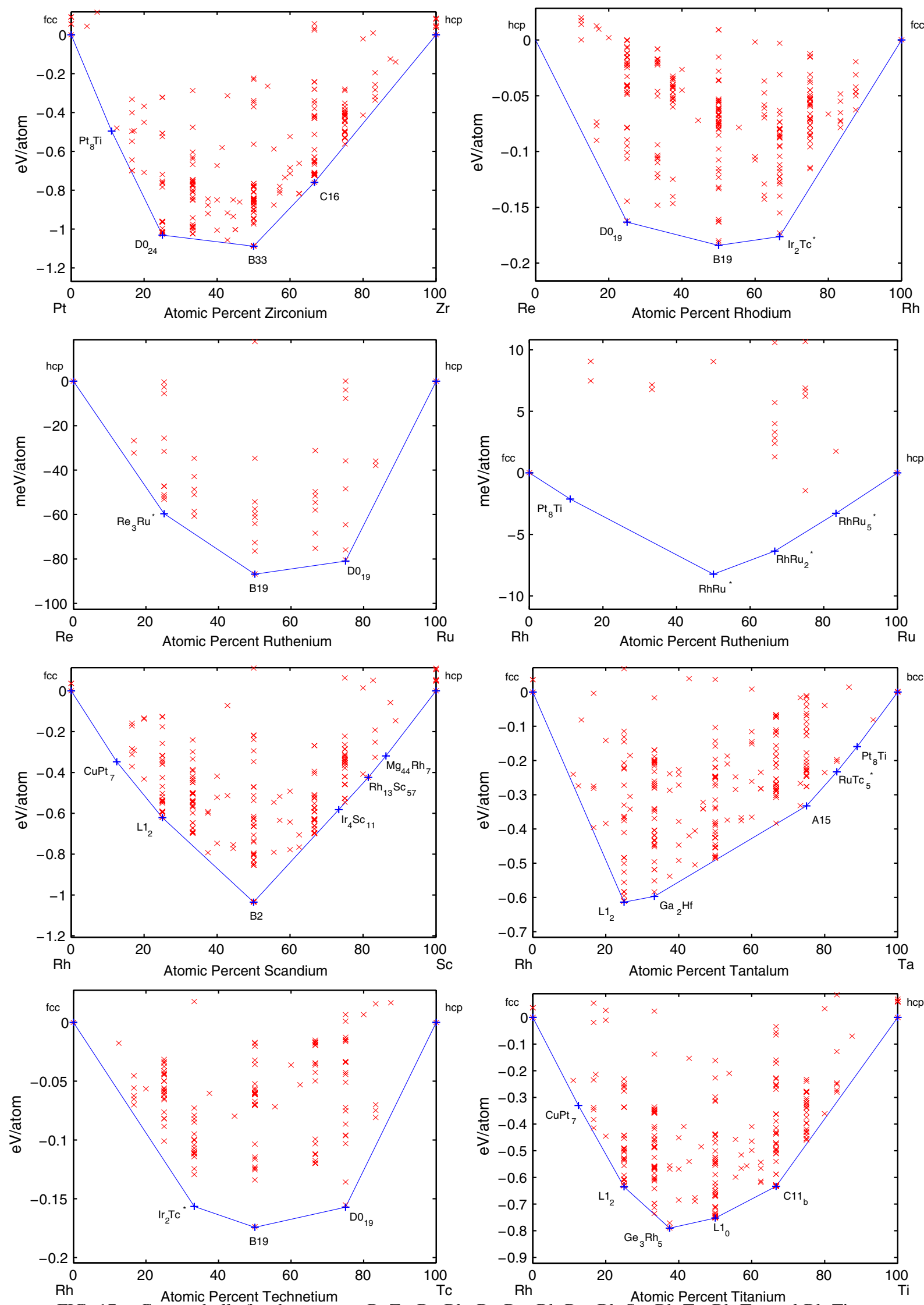

FIG. 17. Convex hulls for the systems Pt-Zr, Re-Rh, Re-Ru, Rh-Ru, Rh-Sc, Rh-Ta, Rh-Tc, and Rh-Ti. 

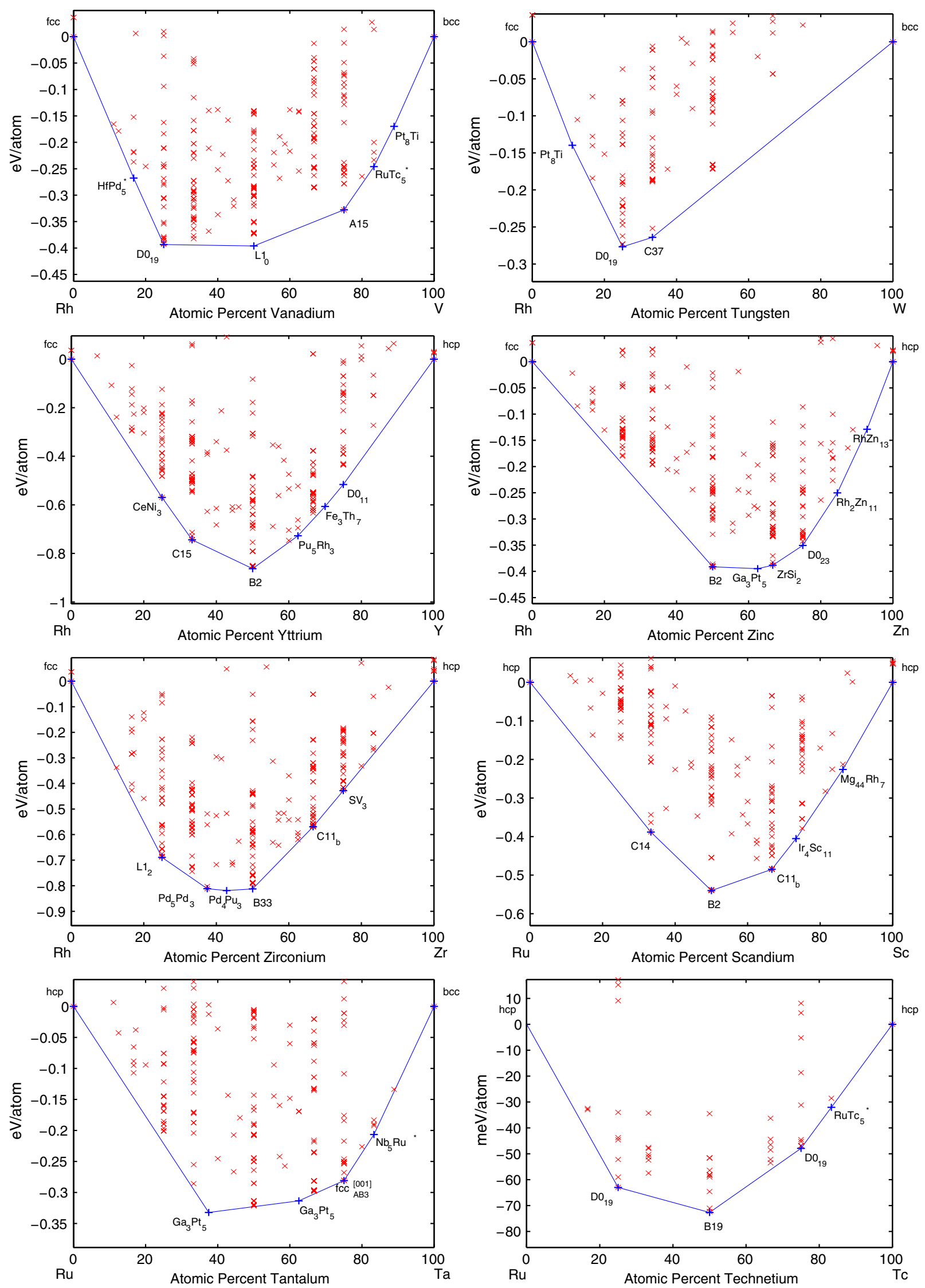

FIG. 18. Convex hulls for the systems Rh-V, Rh-W, Rh-Y, Rh-Zn, Rh-Zr, Ru-Sc, Ru-Ta, and Ru-Tc. 

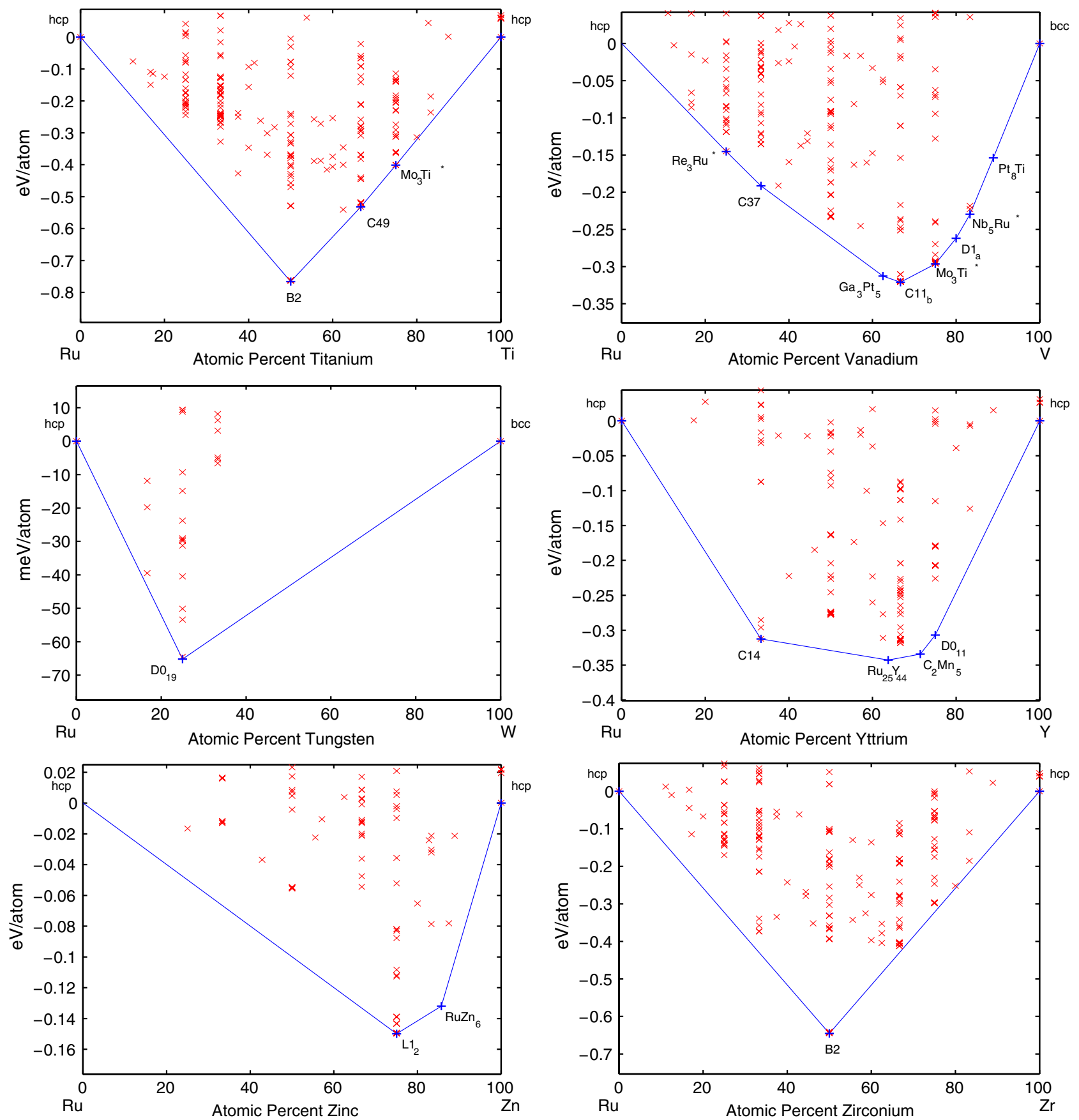

FIG. 19. Convex hulls for the systems Ru-Ti, Ru-V, Ru-W, Ru-Y, Ru-Zn, and Ru-Zr. 
[1] P. J. Loferski, 2008 Minerals Yearbook-Platinum Group Metals (US Geological Survey, Reston, VA, 2008).

[2] S. Curtarolo, D. Morgan, and G. Ceder, Accuracy of ab initio Methods in Predicting the Crystal Structures of Metals: Review of 80 Binary Alloys, CALPHAD: Comput. Coupling Phase Diagrams Thermochem. 29, 163 (2005).

[3] A. V. Ruban, I. A. Abrikosov, and H. L. Skriver, GroundState Properties of Ordered, Partially Ordered, and Random Cu-Au and Ni-Pt Alloys, Phys. Rev. B 51, 12958 (1995).

[4] D. Paudyal and A. Mookerjee, Phase Stability and Magnetism in NiPt and NiPd alloys, J. Phys. Condens. Matter 16, 5791 (2004).

[5] D. Paudyal, T. Saha-Dasgupta, and A. Mookerjee, Phase Stability-Analysis in Fe-Pt and Co-Pt Alloy Systems: An Augmented Space Study, J. Phys. Condens. Matter 16, 7247 (2004).

[6] D. Paudyal, T. Saha-Dasgupta, and A. Mookerjee, Study of Phase Stability in NiPt Systems, J. Phys. Condens. Matter 15, 1029 (2003).

[7] N. A. Zarkevich, T.L. Tan, and D. D. Johnson, FirstPrinciples Prediction of Phase-Segregating Alloy Phase Diagrams and a Rapid Design Estimate of Their Transition Temperatures, Phys. Rev. B 75, 104203 (2007).

[8] S. Bärthlein, E. Winning, G. L. W. Hart, and S. Müller, Stability and Instability of Long-Period Superstructures in Binary Cu-Pd Alloys: A First-Principles Study, Acta Mater. 57, 1660 (2009).

[9] S. Bärthlein, G. L.W. Hart, A. Zunger, and S. Müller, Reinterpreting the $\mathrm{Cu}$-Pd Phase diagram Based on New Ground-State Predictions, J. Phys. Condens. Matter 19, 032201 (2007).

[10] R. Taylor, S. Curtarolo, and G. L. W. Hart, Predictions of the $\mathrm{Pt}_{8} \mathrm{Ti}$ Phase in Unexpected Systems, J. Am. Chem. Soc. 132, 6851 (2010).

[11] M.J. Mehl, G.L. Hart, and S. Curtarolo, Density Functional Study of the $\mathrm{L}_{0}-\alpha \mathrm{IrV}$ Transition in IrV and RhV, J. Alloys Compd. 509, 560 (2011).

[12] R. V. Chepulskii and S. Curtarolo, Revealing LowTemperature Atomic Ordering in Bulk Co-Pt with the High-Throughput ab initio Method, Appl. Phys. Lett. 99, 261902 (2011).

[13] G. L.W. Hart, Verifying Predictions of the $\mathrm{L1}_{3}$ Crystal Structure in Cd-Pt and Pd-Pt by Exhaustive Enumeration, Phys. Rev. B 80, 014106 (2009).

[14] B. Schoenfeld, M. Engelke, and A. V. Ruban, Lack of Support for Adaptive Superstructure $\mathrm{NiPt}_{7}$ : Experiment and First-Principles Calculations, Phys. Rev. B 79, 064201 (2009).

[15] K. Yuge, A. Seko, A. Kuwabara, F. Oba, and I. Tanaka, First-Principles Study of Bulk Ordering and Surface Segregation in Pt-Rh Binary Alloys, Phys. Rev. B 74, 174202 (2006).

[16] S. L. Shang, Y. Wang, D. E. Kim, C. L. Zacherl, Y. Du, and Z. K. Liu, Structural, Vibrational, and Thermodynamic Properties of Ordered and Disordered $\mathrm{Ni}_{1-x} \mathrm{Pt}_{x}$ Alloys from First-Principles Calculations, Phys. Rev. B 83, 144204 (2011).

[17] D. A. Carr, J. Corbitt, G. R. Hart, E. Gilmartin, and G. L.W. Hart, Finding New Phases for Precipitate-
Hardening in Platinum and Palladium Alloys, Comput. Mater. Sci. 51, 331 (2012).

[18] B. Sanyal, S. K. Bose, V. Drchal, and J. Kudrnovsky, Ordering and Segregation in $X \mathrm{Pt}(X=\mathrm{V}, C u$, and $A u)$ Random Alloys, Phys. Rev. B 64, 134111 (2001).

[19] P. E. A. Turchi, V. Drchal, and J. Kudrnovsky, Stability and Ordering Properties offcc Alloys Based on Rh, Ir, Pd, and Pt, Phys. Rev. B 74, 064202 (2006).

[20] M.H.F. Sluiter, C. Colinet, and A. Pasturel, Ab initio Calculation of the Phase Stability in Au-Pd and Ag-Pt Alloys, Phys. Rev. B 73, 174204 (2006).

[21] R. V. Chepulskii, W. H. Butler, A. van de Walle, and S. Curtarolo, Surface Segregation in Nanoparticles from First Principles: The Case of FePt, Scr. Mater. 62, 179 (2010).

[22] L. J. Nelson, S. Curtarolo, and G. L. W. Hart, Ground State Characterizations of Systems Predicted to Exhibit $\mathrm{L}_{1}$ or $\mathrm{L1}_{3}$ Crystal Structures, Phys. Rev. B 85, 054203 (2012).

[23] S. V. Barabash, V. Blum, S. Mueller, and A. Zunger, Prediction of Unusual Stable Ordered Structures of Au-Pd Alloys via a First-Principles Cluster Expansion, Phys. Rev. B 74, 035108 (2006).

[24] O. Levy, R. V. Chepulskii, G. L. W. Hart, and S. Curtarolo, The New Face of Rhodium Alloys: Revealing Ordered Structures from First Principles, J. Am. Chem. Soc. 132, 833 (2010).

[25] M. Jahnatek, O. Levy, G. L. W. Hart, L. J. Nelson, R. V. Chepulskii, J. Xue, and S. Curtarolo, Ordered Structures and Vibrational Stabilization in Ruthenium Alloys from First Principles Calculations, Phys. Rev. B 84, 214110 (2011).

[26] O. Levy, G. L. W. Hart, and S. Curtarolo, Uncovering Compounds by Synergy of Cluster Expansion and HighThroughput Methods, J. Am. Chem. Soc. 132, 4830 (2010).

[27] O. Levy, G. L. W. Hart, and S. Curtarolo, Structure Maps for hcp Metals from First-Principles Calculations, Phys. Rev. B 81, 174106 (2010).

[28] S. Curtarolo, G. L. W. Hart, M. Buongiorno Nardelli, N. Mingo, S. Sanvito, and O. Levy, The High-Throughput Highway to Computational Materials Design, Nat. Mater. 12, 191 (2013).

[29] O. Levy, G. L. W. Hart, and S. Curtarolo, Hafnium Binary Alloys from Experiments and First Principles, Acta Mater. 58, 2887 (2010).

[30] O. Levy, M. Jahnatek, R. V. Chepulskii, G. L. W. Hart, and S. Curtarolo, Ordered Structures in Rhenium Binary Alloys from First-Principles Calculations, J. Am. Chem. Soc. 133, 158 (2011).

[31] O. Levy, J. Xue, S. Wang, G. L. W. Hart, and S. Curtarolo, Stable Ordered Structures of Binary Technetium Alloys from First Principles, Phys. Rev. B 85, 012201 (2012).

[32] A. R. Harutyunyan, E. Mora, T. Tokune, K. Bolton, A. Rosén, A. Jiang, N. Awasthi, and S. Curtarolo, Hidden Features of the Catalyst Nanoparticles Favorable for Single-Walled Carbon Nanotube Growth, Appl. Phys. Lett. 90, 163120 (2007).

[33] S. Curtarolo, W. Setyawan, G. L. W. Hart, M. Jahnatek, R. V. Chepulskii, R. H. Taylor, S. Wang, J. Xue, K. Yang, O. Levy, M. Mehl, H.T. Stokes, D. O. Demchenko, and D. Morgan, AFLOW: An Automatic Framework for 
High-Throughput Materials Discovery, Comput. Mater. Sci. 58, 218 (2012).

[34] S. Curtarolo, W. Setyawan, S. Wang, J. Xue, K. Yang, R. H. Taylor, L. J. Nelson, G. L. W. Hart, S. Sanvito, M. Buongiorno Nardelli, N. Mingo, and O. Levy, AFLOWLIB.ORG: A Distributed Materials Properties Repository from High-Throughput ab initio Calculations, Comput. Mater. Sci. 58, 227 (2012).

[35] P. Villars, M. Berndt, K. Brandenburg, K. Cenzual, J. Daams, F. Hulliger, T. Massalski, H. Okamoto, K. Osaki, A. Prince, H. Putz, and S. Iwata, The Pauling File, Binaries Edition, J. Alloys Compd. 367, 293 (2004).

[36] T. B. Massalski, H. Okamoto, P. R. Subramanian, and L. Kacprzak, Binary Alloy Phase Diagrams (American Society for Metals, Materials Park, OH, 1990).

[37] See www.aflowlib.org.

[38] See the Supplemental Material at http://link.aps.org/ supplemental/10.1103/PhysRevX.3.041035 for the set of geometrical and relaxed structures with $a b$ initio total energies and formation enthalpies.

[39] G. L.W. Hart and R.W. Forcade, Algorithm for Generating Derivative Structures, Phys. Rev. B 77, 224115 (2008).

[40] G. Kresse and J. Hafner, Ab initio Molecular Dynamics for Liquid Metals, Phys. Rev. B 47, 558 (1993).

[41] P.E. Blöchl, Projector Augmented-Wave Method, Phys. Rev. B 50, 17953 (1994).

[42] J. P. Perdew, K. Burke, and M. Ernzerhof, Generalized Gradient Approximation Made Simple, Phys. Rev. Lett. 77, 3865 (1996).

[43] H. J. Monkhorst and J.D. Pack, Special Points for Brillouin-Zone Integrations, Phys. Rev. B 13, 5188 (1976).

[44] D. G. Pettifor, A Chemical Scale for Crystal-Structure Maps, Solid State Commun. 51, 31 (1984).

[45] D. G. Pettifor, The Structures of Binary Compounds. I. Phenomenological Structure Maps, J. Phys. C 19, 285 (1986).

[46] J. K. Stalick, K. Wang, and R. M. Waterstrat, The Crystal Structure and Phase Transition of $\mathrm{Hf}_{2} \mathrm{Pt}_{3}$, J. Phase Equilib. 34, 385 (2013).

[47] G. L. W. Hart and R. W. Forcade, Generating Derivative Structures from Multilattices: Application to hcp Alloys, Phys. Rev. B 80, 014120 (2009).

[48] W. Setyawan and S. Curtarolo, High-Throughput Electronic Band Structure Calculations: Challenges and Tools, Comput. Mater. Sci. 49, 299 (2010).

[49] T. Hahn, International Tables of Crystallography. Volume A: Space-Group Symmetry (Kluwer Academic Publishers,
International Union of Crystallography, Chester, England, 2002).

[50] W. Hume-Rothery, The Metallic State (Oxford University Press, Oxford, UK, 1931).

[51] A.R. Miedema, P.F. de Chatel, and F. R. de Boer, Cohesion in Alloys-Fundamentals of a Semi-empirical Model, Physica (Amsterdam) 100B, 1 (1980).

[52] A. Zunger, Systematization of the Stable Crystal Structure of All AB-Type Binary Compounds: A Pseudopotential Orbital-Radii Approach, Phys. Rev. B 22, 5839 (1980).

[53] D. G. Pettifor, Structure Maps Revisited, J. Phys. Condens. Matter 15, V13 (2003).

[54] P. Villars, K. Brandenburg, M. Berndt, S. LeClair, A. Jackson, Y.H. Pao, B. Igelnik, M. Oxley, B. Bakshi, P. Chen, and S. Iwata, Binary, Ternary and Quaternary Compound Former/Nonformer Prediction via Mendeleev Number, J. Alloys Compd. 317-318, 26 (2001).

[55] C. Wolverton and V. Ozolinš, Entropically Favored Ordering: The Metallurgy of $\mathrm{Al}_{2} \mathrm{Cu}$ Revisited, Phys. Rev. Lett. 86, 5518 (2001).

[56] Fuelling Discovery by Sharing, Nat. Mater. 12, 173 (2013).

[57] R. T. Fielding and R. N. Taylor, Principled Design of the Modern Web Architecture, ACM Trans. Internet Technol. 2, 115 (2002).

[58] A. R. Harutyunyan, N. Awasthi, A. Jiang, W. Setyawan, E. Mora, T. Tokune, K. Bolton, and S. Curtarolo, Reduced Carbon Solubility in Fe Nano-Clusters and Implications for the Growth of Single-Walled Carbon Nanotubes, Phys. Rev. Lett. 100, 195502 (2008).

[59] S. Curtarolo, N. Awasthi, W. Setyawan, A. Jiang, K. Bolton, T. Tokune, and A. R. Harutyunyan, Influence of Mo on the Fe:Mo:C Nanocatalyst Thermodynamics for Single-Walled Carbon Nanotube Growth, Phys. Rev. B 78, 054105 (2008).

[60] F. Cervantes-Sodi, T. P. McNicholas, J. G. S. Jr., J. Liu, G. Csányi, A.C. Ferrari, and S. Curtarolo, Viscous State Effect on the Activity of Fe Nanocatalysts, ACS Nano 4, 6950 (2010).

[61] T. Chookajorn, H. A. Murdoch, and C. A. Schuh, Design of Stable Nanocrystalline Alloys, Science 337, 951 (2012).

[62] H. A. Murdoch and C. A. Schuh, Stability of binary nanocrystalline alloys against grain growth and phase separation, Acta Mater. 61, 2121 (2013).

[63] J. K. Stalick and R. M. Waterstrat, The Hafnium-Platinum Phase Diagram, J. Phase Equilib. (2013). 